\title{
Abstracts for the 42nd Human Genetics Society of Australasia Annual Scientific Meeting
} Sydney, New South Wales

\author{
August 4-7, 2018
}

\section{Poster Presentations}

\section{BIOCHEMICAL GENETICS}

\section{VLCAD DIAGNOSIS BY ENZYME ASSAY}

\section{Devanapalli $B^{\prime}$, Hertzog $A^{\prime}$, Wiley $\mathrm{V}^{\prime}$ \\ ${ }^{I}$ Children's Hospital at Westmead, Westmead, Australia}

Very long chain acylCoA dehydrogenase (VLCAD) deficiency is an inherited disorder of fatty acid oxidation, diagnosed by detecting elevated C14, C14:1 and C12; however, classification is difficult due to molecular heterogeneity. Functional assays including acylcarnitine profiles in fibroblasts (FIBACP) and VLCAD specific enzyme activity are required to clearly identify patients and provide better correlation with the genotype. This report is based on specific enzyme activity as measured by MS/MS compared to the FIBACP profile and any available mutational data. Fibroblast cell lines were tested for VLCAD deficiency on 37 individuals. VLCAD specific enzyme activity was measured in pellets made from cultured fibroblasts. The enzyme assay used ferrocenium hexafluorophosphate as the electron acceptor and $\mathrm{C} 16: \mathrm{CoA}$ as the substrate. Enzyme reactions were performed at $37^{\circ} \mathrm{C}$ for 5 minutes and then quenched with acetonitrile. The products $(\mathrm{C} 16: \mathrm{OHCoA}, \mathrm{C} 16: 1 \mathrm{CoA})$ and substrate $(\mathrm{C} 16: \mathrm{CoA})$ of the enzyme reaction were quantified by MS/MS. Quantitation was based on peak areas of each analyte. Stored cell lines that were previously analyzed by a spectrophotometric method were retested with the current method to develop reference ranges. The activities obtained for our patients were similar to the fibroblast acylcarnitine profile. There were 18 confirmed as having VLCAD deficiency (abnormal FIBACP profile and specific activity $<0.6 \mathrm{nmol} / \mathrm{min} / \mathrm{mg}$ protein). Functional assays of VLCAD activity aid in establishing a genotype-phenotype correlation. This enzymatic method assists in confirming the diagnosis especially if the variant has not been previously described. The enzyme assay provides a reliable result where other molecular and biochemical markers may be inconclusive.

\section{ONE DATA SET, TWO ALGORITHMS}

Lam $K^{\prime}$, Fletcher J', Bonacquisto $L^{2}$, Pitt J ${ }^{2}$, Metz $M^{\prime}$, Ranieri E

${ }^{I}$ SA Pathology, Australia

2 Victorian Clinical Genetics Services, Australia

Aim: To compare the first trimester Down syndrome (Trisomy 21) risk assessment from one data set by two different software algo- rithms. Introduction: The South Australian Maternal Serum Antenatal Screening (SAMSAS) software program generates risk assessment using a SAMSAS developed algorithm. Utilising maternal serum markers, placenta associated plasma protein A (PAPPA), free beta human chorionic gonadotropin ( $\beta \mathrm{hCG}$ ) and ultrasound Nuchal Translucency with covariance of maternal age, weight and ethnicity. The performance of the SAMSAS-developed software is compared to the commercially available LMS ALPHA software. Method: A data set of 5527 of maternal sera included gestational age from 9-14 weeks gestation, measurement of maternal serum markers PAPP- A, free $\beta \mathrm{hCG}$, nuchal translucency and maternal weight. We determined the antenatal aneuploidy risk using the SAMSAS algorithm and compared that to the LMS ALPHA software-determined risk. Results: In the cohort of 5,527 samples, using a cut-off risk for aneuploidy set at 1 in 250, the LMS ALPHA software identified 175 cases at increased risk for trisomy 21. SAMSAS software identified 203 cases. Cases that were identified at increased risk were recommended for invasive testing, as per screening protocol. A total of 8 cases were confirmed as trisomy 21 affected pregnancy by cytogenetic karyotype results. Both laboratories achieved $100 \%$ detection rate with a false positive rate of $3.17 \%$ for LMS Alpha and $3.67 \%$ for SAMSAS software respectively. Conclusion: SAMSAS risk aneuploidy generation is equivalent to that provided by LMS ALPHA, noting that there were cases that showed discordant risk assessment.

\section{CANCER GENETICS}

3. HEREDITARY LEIOMOYOMATOSIS AND RENAL CELL CARCINOMA SYNDROME AN EFFECTIVE APPROACH TO A HEREDITARY CANCER SYNDROME

Al-Shinnag $\mathrm{M}^{1,2,3}$, Marfan $\mathrm{H}^{2,3}$, Susman $\mathrm{R}^{2,3}$, Wakeling $\mathrm{J}^{3}$, Wood $\mathrm{S}^{4}$, Mallett $\mathrm{A}^{1,2}$

${ }^{I}$ Kidney Health Service, Royal Brisbane and Women's Hospital, Herston, Australia

${ }^{2}$ Faculty of Medicine, The University of Queensland, Herston, Australia

${ }^{3}$ Genetic Health Queensland, Herston, Australia

${ }^{4}$ Department of Urology, Princess Alexandra Hospital, Woolloongabba, Australia

Background: HLRCC is an autosomal dominant disorder caused by FH mutations characterized by skin piloleiomyomas, uterine leiomyomas and RCC. The cumulative risk of RCC is $10-16 \%$ 
with mean age at first RCC diagnosis of 41 years. Life-long RCC screening recommended. Aim: To assess adherence to surveillance guidelines in an Australian HLRCC cohort, and to describe disease characteristics. Method: All patients with a diagnosis (clinical/genetic) of HLRCC at RBWH from 01/01/2014-31/12/2017 were included (HREC/17/QRBW/276). All patients were assessed and counselled by a Clinical Geneticist then referred to an Adult Nephrologist. Baseline and incident clinical variables were extracted and analyzed. Results: 22 patients were identified with median age of 57 years. 16/22 were female. The median and cumulative follow-up time were 2 and 37 years respectively. Cutaneous leiomyomas were noted in $15 / 22$, cutaneous leiomyosarcoma in $1 / 22$, and uterine fibroids in 10/16 females. RCC was diagnosed in $1 / 22$ during surveillance (age 47 years) with a second patient awaiting surgical intervention for potential RCC (age 66 years). 4/22 had RCC diagnosed prior to commencement of surveillance (ages 11-43 years). Surveillance MRI occurred in 19/22 patients, $1 / 22$ was lost to follow up before first MRI, 1/22 is yet to have first MRI and 1/22 has experienced logistic difficulties. 18/22 have regular annual review. Of the remaining, 2/4 have been lost to follow-up and 2/4 have been onward referred for follow up by their regional renal service. Conclusion: Evidence-based RCC screening is feasible and able to identify incident renal lesions. Multidisciplinary patient management enables expedited genetic counseling, diagnosis, longitudinal screening and RCC management.

\section{BIRT HOGG DUBÉ SYNDROME (BHD): AN EFFECTIVE MULTIDISCIPLINARY APPROACH TO A HEREDITARY RENAL CANCER SYNDROME}

\author{
Al-Shinnag $M^{1,2,3}$, Marfan $\mathrm{H}^{2,3}$, Susman $\mathrm{R}^{2,3}$, Wakeling $\mathrm{J}^{3}$, Wood $\mathrm{S}^{4}$, Mallett $\mathrm{A}^{1,2}$ \\ ${ }^{I}$ Kidney Health Service, Royal Brisbane and Women's Hospital, Herston, Australia \\ ${ }^{2}$ Faculty of Medicine, The University of Queensland, Herston, Australia \\ ${ }^{3}$ Genetic Health Queensland, Herston, Australia \\ ${ }^{4}$ Department of Urology, Princess Alexandra Hospital, Woolloongabba, Australia
}

Background: BHD is a rare autosomal dominant disorder caused by germline FLCN mutations characterized by skin fibrofolliculomas, lung cysts, spontaneous pneumothorax and renal cell carcinoma (RCC). The cumulative risk of RCC is $16 \%$ with lifelong screening recommended. Aim: To assess adherence to surveillance guidelines in an Australian BHD cohort and to describe disease characteristics. Method: All patients with a diagnosis (clinical/genetic) of BHD at RBWH 01/01/2014-31/12/2017 were included (HREC/17/QRBW/276). All patients were initially assessed and counselled by a Clinical Geneticist and then referred to an Adult Nephrologist. Baseline and incident clinical variables were extracted and analyzed. Results: 18 patients were identified with a median age of 57 years. 9/18 were female. The median and cumulative follow-up times were 1.5 years and 39 years respectively. Cutaneous fibrofolliculoma was noted in $8 / 18$ patients, lung cysts in $3 / 18$ patients, spontaneous pneumothorax in $3 / 18$ patients, and past parotid oncocytoma in $2 / 18$. A positive family history was documented in $12 / 18$ patients. Chest X-ray or CT was undertaken in $13 / 18$ patients. Surveillance MRI occurred in $13 / 18$ patients, initially annually and now third yearly. $5 / 18$ patients who hadn't undertaken surveillance imaging: $3 / 5$ yet to have MRI and $2 / 5$ lost follow up. 2/18 patients have been diagnosed with RCC at the ages of 73 and 77 years, both occurring prior to surveillance. No RCC events have occurred while under surveillance; however, 2/18 are undergoing intensified imaging for renal lesions of uncertain significance. Conclusion: Coordinated screening and longitudinal care for BHD via multidisciplinary collaboration is feasible, with especial attention warranted for surveillance guideline adherence.

\author{
5. SURVEILLANCE IN INDIVIDUALS WITH HIGH \\ MULTI-ORGAN CANCER RISK

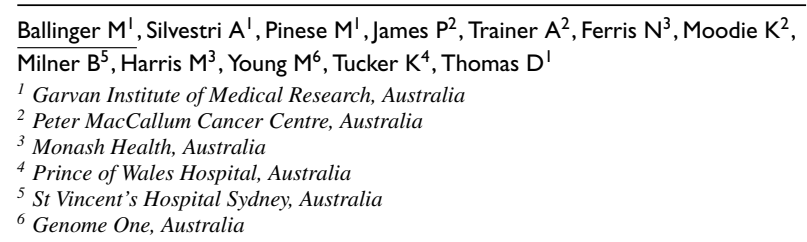

Background: Comprehensive cancer risk management guidelines for individuals at high multi-organ cancer risk are lacking. Research studies support whole body MRI as part of a baseline surveillance assessment. Aims: To estimate the prevalence and incidence of investigable lesions in a high-risk population and investigate mutational signatures in peripheral blood as an early detection mechanism; and to assess the acceptability, psychosocial impact and cost effectiveness of the surveillance program. Methods: The surveillance program includes annual whole-body MRI, physical examination, full blood evaluation, fecal occult blood testing, breast MRI and 2-5 yearly colonoscopy/endoscopy. Deep sequencing will be used to detect genetic signatures of malignancy in cell free plasma. Psychometric evaluations are undertaken and participants complete cost diaries. Medicare/PBS data is also utilized. Results: Since July 2013, 42 TP53 pathogenic variant carriers (25F, 17M; age 18-63 years) have been enrolled. After completion of 38 first-year whole body MRI scans, three new asymptomatic malignancies (lumbar liposarcoma, Gleason score 8 and 9 prostate cancers) have been detected. Second- and third-year whole body MRI scans (45 total) detected an additional four new primary cancers; thymic pleomorphic liposarcoma, pelvic chondrosarcoma, renal leiomyosarcoma and a pheochromocytoma. All other screening procedures have been normal. Mutational signatures of malignancy in the peripheral blood were detectable by sequencing. Preliminary psychosocial data indicate no lasting adverse effects. Conclusion: Evaluation of the surveillance schedule is ongoing. Detection of seven asymptomatic primary malignancies (treated with curative intent), in conjunction with the acceptability and lack of negative psychological impact supports evaluation over a longer term.

\section{MAINSTREAM BRCA1/2 TESTING FOR BREAST CANCER: THE PARKVILLE FAMILIAL CANCER CENTRE EXPERIENCE}

Beard $C^{\prime}$, Cicciarelli $L^{\prime}$, Kentwell $M^{\prime}$, Monohan $K^{\prime}$, Hodgkin $L^{\prime}$, Sexton $A^{\prime}$, James $P^{1}$

${ }^{I}$ Parkville Familial Cancer Centre, Melbourne, Australia

In recent years, genetic testing for the hereditary breast/ovarian cancer genes BRCA1 and BRCA2 has become an important part of informing treatment decisions for a subset of women diagnosed with breast cancer. In this time, Familial Cancer Centres (FCCs) have experienced an influx of treatment-focused referrals with a time pressure to obtain genetic test results. For patients where a BRCA1/2 gene test result is going to help inform their treatment, the primary focus of the genetic testing becomes about the patient's ongoing treatment with their specialist, with the familial implications of testing becoming secondary. Traditionally, genetic testing for hereditary cancer genes has been performed by genetic health professionals in an FCC. Mainstream genetic testing is an alternative model of care, where a cancer patient is offered genetic testing from their oncology specialist directly, with support from an FCC to follow up the familial implications of the result. The Parkville Familial Cancer Centre rolled out a mainstream genetic testing program for a subset of breast cancer patients at their clinical sites in Victoria in September 2017. Oncology/surgical/radiation specialists in the Breast Clinics at the Victorian sites were upskilled prior to the roll-out of the 
program. The program was developed using experience from the ovarian mainstreaming genetic testing program in Australia, our local gynecology mainstreaming model, as well as international experience. We plan to present the program's protocol, as well as an audit of the program so far, with insights into some of the benefits and challenges of the program.

\section{DELAYED DIAGNOSIS OF PALLISTER-HALL SYNDROME IN ADULTHOOD FOLLOWING THE INCIDENTAL DETECTION OF A HYPOTHALAMIC HAMARTOMA}

\section{Courtney $\mathrm{E}^{\prime}$, Soon $\mathrm{S}^{2}$, Ishak $\mathrm{D}^{\prime}$, Ngeow J $\mathrm{J}^{1,3,4,5}$}

${ }^{I}$ Cancer Genetics Service, Division of Medical Oncology, National Cancer Centre Singapore, Singapore

${ }^{2}$ Department of Endocrinology, Singapore General Hospital, Singapore

${ }^{3}$ Lee Kong Chian School of Medicine, Nanyang Technological University, Singapore

${ }^{4}$ Oncology Academic Clinical Program, Duke-NUS Medical School, Singapore

${ }^{5}$ Institute of Molecular and Cell Biology, Agency for Science Technology and Research ( $A *$ Star), Singapore

Background: Pallister-Hall syndrome (PHS) is a rare autosomal dominant condition that is associated with polydactyly and hypothalamic hamartoma and is caused by predominantly frameshift or nonsense pathogenic variants in the GLI3 gene. It is typically identified during childhood; however, rare reports of diagnoses during adulthood exist. Here we describe the identification of a novel nonsense GLI3 pathogenic variant in an adult following the incidental detection of a hypothalamic hamartoma. Case: A 21-year-old Chinese male who was undergoing MRI for investigation of mild-moderate hearing loss, was incidentally found to have a hypothalamic hamartoma. He was born with bilateral polydactyly and prognathism, was developmentally delayed and had precocious puberty at age 8 . The patient reported previous episodes of limb jerking, but multiple neurology assessments have been normal. Multi-gene panel testing revealed a novel heterozygous nonsense pathogenic variant in GLI3, NM_000168.5:c.2071C > T p.(Gln691*). He has no family history of PHS, and the GLI3 pathogenic variant was not detected in his parents or only sibling, suggesting it was de novo (although germline mosaicism cannot be excluded). Conclusion: This case adds to the literature of PHS diagnosed in adulthood, which together serve to bring attention to the missed opportunities for genetic testing and subsequent diagnosis. Earlier diagnosis has the potential to alleviate the burden on patients and their families when there is a lack of a definitive reason for their medical history. This is especially important for PHS manifestations where early detection and management can help improve outcomes, such as developmental and endocrine related features.

\section{WHY DO PATIENTS CHANGE THEIR MIND ABOUT CANCER GENE TESTING?}

\section{Gattas $M^{1}$, Welch $A^{2}$ \\ ${ }^{1}$ Brisbane Genetics, Auchenflower, Australia \\ ${ }^{2}$ University of Qld, St Lucia, Australia}

Patients who had been seen for genetic counseling in a private genetics clinic and had signed consent for cancer gene panel testing but had not followed through to have the test were the focus of this study. All the patients were seen prior to 1 July 2017. Patients were contacted by telephone in January 2018 and invited to participate in a 7-question survey over the phone to ask why they had not had a cancer gene test. This meant that at least six months had elapsed since the time of their appointment. Results: Attempts were made to contact 59 patients, 36 patients could not be contacted, 2 patients did not want to participate, 1 had completed testing as a direct to consumer test, and 20 patients completed the survey. The commonest reason patients decided not to have the test was a conscious decision that it was not of benefit to them. This was reported in $12 / 20$. The next most common was concern the result may affect their life insurance or their children's life insurance in 11/20. Concern that the result may affect medical insurance was reported in $6 / 20$ patients. At least one patient did follow through with the test after completing the survey and was found to be a MUTYH mutation carrier.

\section{EXPLORING THE BARRIERS PREVENTING INDIGENOUS AUSTRALIANS FROM ACCESSING CANCER GENETIC COUNSELING}

Gonzalez $C^{\prime}$, Williams $R^{2}$, Fleming J', Barlow-Stewart $K^{\prime}$, Wadwell $R^{3}$, Harris $R^{4}$, Buckman $\mathrm{M}^{5}$

${ }^{1}$ Discipline of Genetic Medicine, University of Sydney Medical School - Northern, St Leonards, Australia

${ }^{2}$ Prince of Wales Hereditary Cancer Centre, Prince of Wales Hospital, Randwick, Australia

${ }^{3}$ Aboriginal Health Unit, Tamworth Rural Referral Hospital, Tamworth, Australia ${ }^{4}$ Westmead Familial Cancer Service, Sydney, NSW, Australia

${ }_{5}^{5}$ Tamworth Genetic Counselling Service, Tamworth Rural Referral Hospital, Tamworth, Australia

Background: The Hunter New England Local Health District has the largest Aboriginal population of the NSW health districts. However, few Aboriginal clients attend the Tamworth Cancer Genetic Counselling service. While the literature suggests that Aboriginal clients are interested in discussing familial cancer, no research has explored the cultural issues impacting service attendance. Aims: (1) To explore the attitudes of Aboriginal people in Tamworth towards cancer and the barriers preventing access to cancer genetic counseling. (2) To explore the views of Aboriginal Health Workers (AHWs) and researchers working with Indigenous communities, and compare their perspectives to the community in Tamworth. Methods: A focus group with an Aboriginal Elders group in Tamworth is being conducted. The semi-structured focus group guide has been informed by the community consultation undertaken in the preparation for ethics submissions with the AH\&MRC. As the community does not wish to be audio recorded or have notes taken during the meeting, notes will be taken afterwards. Health researchers and AHWs ( $\sim 15$ participants) will then be recruited for audio-recorded telephone interviews. The interview guide will be informed by findings from the focus group. All transcripts will be coded and analyzed using thematic analysis. Results: We will present the results of the focus group and interviews, which will provide insight on the issues faced by Indigenous communities when accessing cancer genetic counseling. Conclusion: Identification of barriers impacting on Indigenous clients accessing cancer genetic may assist the services to provide more inclusive health care to the Indigenous community.

\section{BREAST CANCER AND POLYGENIC RISK: CHANGING THE DYNAMIC OF COMMUNICATING GENETIC INFORMATION}

Gregory $G^{\prime}$, Yanes $T^{2,3}$, Meiser $B^{2}$, Barlow-Stewart $K^{\prime}$, Fleming J', Young $M^{4}$, ames $\mathrm{P}^{\mathrm{5}}$

${ }^{1}$ Discipline of Genetic Medicine, University of Sydney Medical School - Northern, St Leonards, Australia

${ }^{2}$ Prince of Wales Clinical School, Faculty of Medicine, UNSW Sydney, Sydney, Australia

${ }^{3}$ School of Psychiatry, Faculty of Medicine, UNSW Sydney, Sydney, Australia ${ }^{4}$ Garvan Institute of Medical Resarch, Darlinghurst, Australia

${ }_{5}^{5}$ Parkville Integrated Familial Cancer Centre, Peter MacCallum Cancer Centre, Melbourne, Australia

Polygenic risk scores (PRS) are fast proving their clinical validity as an important assessment tool for familial breast cancer risk. However, the communication of PRS has not been studied. This study explored the communication behaviors of genetic health professionals returning PRS results compared to previously reported genetic counseling in the familial cancer clinic for monogenic disorders. Participants were recruited from the Variants in Practice (ViP) study and included affected or unaffected women with a high-risk family history of breast cancer and no BRCA1/2 mutation. Sixty-two SNPs were sequenced and PRS determined. Participants returned to the clinic to discuss their PRS and impact on their current breast cancer 
risk. The consultations conducted by genetic health professionals, trained to provide PRS, were audio-recorded. Interviews are being coded to enable analyses of communication behaviors to facilitate comparison to the previous study. Twenty-two face-to-face consultations were conducted by five genetic professionals and are being analyzed to compare different types of information giving and process behaviors. Preliminary findings note differences styles of engagement which may affect participant understanding of PRS. This highlights the importance of education strategies for genetic professionals to assist the effective communication of new technologies. The inclusion of PRS in the hereditary breast cancer clinic will bring a new dynamic to genetic counseling practice. It is important to assess how such developments can be smoothly incorporated into existing practice, and how they may affect the communication behaviors of genetic professionals.

\section{RELEVANCE OF FUNCTIONAL SUBTYPING OF IKZF1 DELETION-LOSS-OF-FUNCTION OR DOMINANT-NEGATIVE, IN PEDIATRIC B-CELL ACUTE LYMPHOBLASTIC LEUKEMIA}

\author{
Gupta $S^{\prime}$, Bakhshi $S^{\prime}$, Kamal V² \\ ${ }^{I}$ All India Institute of Medical Sciences (AIIMS), New Delhi, India \\ ${ }^{2}$ National Institute of Epidemiology, Chennai, India
}

Background: IKZF1 deletions occur in $15-20 \%$ of pediatric Bcell Acute Lymphoblastic Leukemia (B-ALL) and are associated with poor outcome. Novel therapies like retinoids or casein kinase inhibitors hold promise for these patients. Recently, it was shown that only loss-of-function but not dominant-negative type of IKZF1 deletions are associated with adverse prognosis in adult BCR-ABL1-negative B-ALL. We assessed the distribution and clinical impact of functional subtypes of IKZF1 deletions in pediatric B-ALL. Methods: The genomic DNA from pediatric B-ALL cases over 24 months was analyzed for IKZF1 deletions using multiplex ligation dependent probe amplification. The deletions were divided into functional subgroups-A) Loss-of-function (whole gene deletions or those involving exon 2), B) Dominant negative (exon 4-7 deletions), C) others. Results: Overall 35 (19.6\%) of 179 pediatric B-ALL, median age 6 years (2 months-18 years), had IKZF1 deletions. This included $26(15.6 \%)$ of 167 BCR-ABLnegative and $9(75 \%)$ of 12 BCR-ABL1-positive cases. Twentytwo $(85 \%)$ of 26 IKZF1 deletions in BCR-ABL1-negative group were loss-of-function, 2-dominant negative, 2-others. Five of 9 in BCR-ABL1-positive group were loss-of-function and 4-dominant negative deletions. The BCR-ABL1-negative group with loss-offunction IKZF1 deletions $(n=22)$ had lower post-induction remission rates $(72.2 \%$ vs $86.6 \% ; p=.111)$, significantly worse eventfree-survival $(p=.011)$ and overall-survival $(p=.004)$ compared to cases without IKZF1 deletions $(n=141)$. Conclusions: The IKZF1 deletions were seen in $19.6 \%$ pediatric B-ALL. Most of the IKZF1 deletions in BCR-ABL1-negative patients were loss-offunction type, which had worse outcome compared to cases without IKZF1 deletions. Such cases may be investigated further for novel alternative therapies.

\section{APPLICATION OF PLASMA CELL-ENRICHED FISH ASSAY ENHANCES ABNORMALITY DETECTION RATES IN PLASMA CELL DYSCRASIA (PCD)}

Ho J', Lim $T^{\prime}, \operatorname{Lim} A^{\prime}$, Lau $L^{\prime}$, Yeap $Y^{\prime}, N g Y^{\prime}$, What $K^{\prime}$, Chen $Y^{2}$, Tien $S^{1,2}$

${ }^{1}$ Department of Molecular Pathology, Singapore General Hospital, Singapore

${ }^{2}$ Department of Haematology, Singapore General Hospital, Singapore

Background: Cytogenetic abnormalities in PCD, detectable with fluorescence in-situ hybridization (FISH) and karyotyping on plasma cells (PC), are essential for prognostic stratification and patient treatment. Low PC levels in bone marrow aspirates may reduce FISH assay sensitivity. Aim: To validate clinical utility of PC enrichment for FISH assays in PCD patients. Materials and Methods: 126 suspected PCD cases were enriched for CD138+ PCs using magnetic cell sorting, followed by FISH assays using DNA probes for 1q21, FGFR3/IGH, CCND1/IGH, RB1, IGH/MAF, TP53, CEP9, CEP11 and CEP15. Results were correlated with karyotype and BMA/flow cytometry findings. Risk group stratification was based on the cytogenetic results obtained. Results: Of 126 cases, 27 were non-PCD cases and 99 confirmed-PCD cases. All 27 non-PCD cases were FISH-negative with normal karyotypes except for 3 myeloid disorders revealing $\mathrm{t}(\mathrm{Y} ; 1)$, trisomy 10 and deletion 20q. All 99 confirmedPCD cases were FISH-positive but only $54.5 \%$ (54/99) had abnormal karyotypes. Based on the FISH results, the patients were stratified into poor, intermediate and favourable prognostic groups comprising $54.5 \%(54 / 99), 15.2 \%$ (15/99) and 30.3\% (30/99), respectively. FISH further delineated the confirmed-PCD cases with normal karyotypes $(n=45)$ into poor $(57.8 \%, 26 / 45)$, intermediate $(17.8 \%, 8 / 45)$ and favourable $(24.4 \%, 11 / 45)$ prognostic groups. Conclusion: The cytogenetic abnormality detection rate reached $100 \%$ with PC-enriched FISH assays compared to $54.5 \%$ by karyotyping. More importantly, FISH reclassified a significant $57.8 \%$ of cases reported with a normal karyotype as having poor prognoses, and therefore therapeutic implications. PC enrichment enhances the FISH abnormality detection rate in patients with PCD.

\section{UNDERSTANDING AND EXPERIENCES OVER TIME OF WOMEN UNDERGOING GENETIC TESTING FOR NEWLY DIAGNOSED BREAST CANCER}

\author{
Jacobs $C^{1,2,3}$, Smith J ${ }^{4}$, Michie $S^{3}$ \\ ${ }^{I}$ UTS, Sydney, Australia \\ ${ }^{2}$ UNSW, Sydney, Australia \\ ${ }^{3}$ UCL, London, UK \\ ${ }^{4}$ Birkbeck, University of London, UK
}

Background: Although genetic testing is increasingly undertaken shortly after breast cancer diagnosis, little is known about women's understanding and experiences of genetic testing during this time. Aim: This longitudinal study aimed to explore the understanding and experiences of women with newly diagnosed breast cancer before genetic counseling, after genetic testing and after results disclosure. Methods: Using Interpretative Phenomenological Analysis, in-depth, semi-structured interviews were undertaken with 11 participants at three time points. Participants were stratified into four groups for analysis according to their expectation of inheritance and their genetic test result. Results: Regardless of the genetic test result, those who expected hereditary cancer consistently felt that the cancer was due to a hereditary cause. Those who did not expect hereditary cancer experienced growing uncertainty about the possibility of inheritance, even when no variant was detected. Where the genetic test result was congruent with the expectation of inheritance, participants experienced growing confidence in decisions about cancer surgery. Where the genetic test result was contrary to expectations, participants experienced growing concern about surgical decisions. Regardless of the result, those who expected hereditary cancer consistently experienced feelings of responsibility for the family. Those with no prior expectation of inheritance experienced growing relief from responsibility for the family when no pathogenic variant was detected. Participants with a pathogenic variant or VUS experienced dissatisfaction with health professionals' communication, while those without remained satisfied. Discussion: Eliciting expectations of inheritance may help to identify patients who would benefit from post-test genetic counseling and support, regardless of the result. 


\section{DIMINISHED ATM FISH SIGNALS IN CLL PATIENTS REQUIRE FURTHER INVESTIGATION TO PROVIDE IMPORTANT PROGNOSTIC INFORMATION}

Farrell $D^{\prime}$, Beddow $R^{\prime}$, Jay $A^{\prime}$, Brown $A^{\prime}$

${ }^{l}$ Wellington Regional Genetic Services, Newtown, New Zealand

Cytogenetic analysis is essential for the diagnosis and prognosis of hematopoietic neoplasms in current clinical practice. Chromosomal abnormalities in chronic lymphocytic leukemia (CLL) are detected by fluorescent in situ hybridization (FISH) in up to $80 \%$ of patients. The cytogenetic abnormalities found include deletions of 11q (ATM), 13q (D13S319), 17p (TP53) and trisomy 12. Deletions of ATM and TP53 in CLL are associated with poor prognostic outcomes and may indicate disease progression. We present a case where a diminished ATM FISH signal, led to further investigation and a subsequent deletion of ATM was confirmed by microarray analysis (aCGH). The prognostic significance of these findings highlights the importance of further testing with more sensitive molecular methods for accurate cytogenetic reporting.

\section{CLINICAL AUDIT OF ALL CHILDREN REFERRED TO THE PEDIATRIC ONCOLOGY GENETIC CLINIC AT GSWA}

\section{Pathak $\mathrm{G}^{\prime}$, Kiraly-Borri $\mathrm{C}^{\prime}$, Townshend $\mathrm{T}^{\prime}$, Pachter $\mathrm{N}^{\prime}$ \\ ${ }^{1}$ Genetic Services of Western Australia, King Edward Memorial Hospital, Perth, Australia}

Background: To address the particular needs of families affected with childhood cancer, identify at risk siblings/parents, and increase the awareness of pediatric cancer predispositions a dedicated pediatric oncology genetic pathway was created at GSWA. The pathway includes 3-4 monthly multidisciplinary meetings, a specific referral form for pediatric oncologists, priority triage based on clinical indications and particular family situation and timely genetic appointments at the genetic clinic or in the oncology ward. Aims of this audit are to identify: (1) number of children referred to and seen through this pathway (Jan 2015-March 2018); (2) time frame between the referral and the appointment; (3) the yield of genetic testing in our setting; (4) number of at-risk siblings who obtained genetic testing and/ or risk management. Methods: A retrospective audit will be conducted, collecting data using the online patient records software, hospital charts and minutes from the genetic-pediatric oncology meetings. The data will be analyzed and results will then be compared the pediatric oncology patients referred to GSWA before this pathway was created. Significance: This audit will provide useful insights in: (1) identifying children with inherited cancer predispositions and their at risk siblings through the creation of first multidisciplinary surveillance clinic for at risk children; (2) long-term follow-up of pediatric cancer patients and their siblings; (3) potential benefits of a dedicated pediatric oncology genetic pathway.

\section{GENETIC INSIGHTS INTO HEREDITARY CANCER RISK} IN THE GLOBAL POPULATION

Servais $L^{\prime}$, Zimmer $A^{\prime}$, McFadden $W^{\prime}$, Gattas $M^{2}$, Gardner $A^{\prime}$, Ryan $L^{\prime}$, Rugeles $J^{3}$, Johnson $C^{4}$, Mokbel $K^{5}$, Evans $D^{6}$, Okazaki $Y^{7}$, Garcia-Castillo $H^{8}$, Boutis $A^{9}$, Bernath $\mathrm{V}^{10}$, Maqueda $\mathrm{C}^{11}$, Gomes $\mathrm{M}^{12}$, Neffa $\mathrm{F}^{13}$

${ }^{1}$ Color, Burlingame, United States

${ }^{2}$ Brisbane Genetics, Auchenflower, Australia

${ }^{3}$ IMAT, Montería, Colombia

${ }^{4}$ University of Calgary, Calgary, Canada

${ }^{5}$ The London Breast Institute, The Princess Grace Hospital, London, Great Britain

${ }^{6}$ University of Manchester, Manchester, Great Britain

${ }^{7}$ Rainbow Genomics, Hong Kong, Hong Kong

${ }^{8}$ Vida en Genoma, Mexico City, Mexico

${ }^{9}$ Theagenio Cancer Hospital, Thessaloniki, Greece

${ }^{10}$ Genda Molecular Biology Laboratory, Buenos Aires, Argentina

${ }^{11}$ Laboratorio Dr Grasa, Zaragoza, Spain

${ }^{12}$ Universidade Federal Fluminense and Universidade Estadual do Rio de Janeiro, Rio de Janeiro, Brazil

${ }^{13}$ Laboratorio Genia, Montevideo, Uruguay

Background: Current hereditary cancer risk data is mostly based on affected Caucasian or Ashkenazi Jewish individuals. As a result, the distribution of genetic mutations and their associated cancer risk in other populations is not well understood. Aim: To provide insights into genetic cancer risk within multiple global populations for breast, ovarian, colorectal, melanoma, pancreatic, prostate, uterine and stomach cancers. Methods: We describe the demographics and results of 8,243 individuals from 17 countries (Argentina, Australia, Belgium, Brazil, Canada, Colombia, Spain, Finland, United Kingdom, Greece, Hong Kong, Ireland, Israel, Japan, Mexico, Peru, Uruguay) who received the physician ordered 30gene Color Hereditary Cancer Test to assess their risk for hereditary cancer. Results: Overall positive rate in the cohort was $16.8 \%$, and varied by country: Brazil 21.5\% (146/532), Greece $21.5 \%$ (97/355), Colombia 21.1\% (136/508), Mexico 20.2\% (108/426), Argentina $19.5 \%$ (82/339), Canada $17.9 \%$ (241/1103), Uruguay $16.5 \%$ (55/278), Australia 16.2\% (142//734), Finland 15.9\% (17/90), Israel $15.8 \%$ (179/953), Peru 15.5\% (20/109), Ireland $12.8 \%$ (20/136), Belgium 12.0\% (26/190), Japan 11.5\% (11/85), United Kingdom 9.6\% (73/690), Spain 8.2\% (12/135), Hong Kong 7.9\% (17/198). The percent positive by ethnicity was: Hispanic $21.1 \%$, Ashkenazi Jewish $17.7 \%$, African $17.1 \%$, Caucasian $16.8 \%$, Native American $16.3 \%$, Asian $11.6 \%$, multiple ethnicity $10.5 \%$, and no answer 15.9\%.Discussion: These data represent various approaches to genetic testing; some focus more on affected individuals or high-risk families and some offer testing to a broader population, resulting in a range of pathogenic variants identified.

\section{MOLECULAR PROFILING OF FFPE OVARIAN TUMOR TISSUE: ASSAY VALIDATION AND PILOT STUDY}

Taylor $\mathrm{P}^{1}$, Kovalenko $\mathrm{S}^{\prime}$, Crooks $\mathrm{R}^{\prime}$, Provan $\mathrm{P}^{2,3}$, DeFazio $A^{2,3}$, Kennedy $\mathrm{B}^{\prime}$, Friedlander $M^{4,5}$, Caramins $M^{1,5}$

${ }^{1}$ Genomic Diagnostics, Heidelberg, Australia

${ }^{2}$ The Westmead Institute for Medical Research, The University of Sydney, Sydney, Australia

${ }^{3}$ Westmead Hospital, Sydney, Australia

${ }^{4}$ Royal Hospital for Women, Sydney, Australia

${ }^{5}$ University of New South Wales, Sydney, Australia

With the introduction of Poly(ADP-ribose) polymerase inhibitors, a novel and effective targeted treatment has become available for women with recurrent ovarian cancers and inactivating BRCA1 or BRCA2 variants. The utility of diagnostic testing for germline BRCA1/2 mutations in ovarian cancer is well established; testing assists in establishing causation, prognosis, and treatment options, future cancer risk and cancer risk reduction in affected family members. There is increasing evidence supporting the clinical importance of testing for somatic BRCA mutations in ovarian cancers, but there are no Australian publications quantifying additional yield or clinical utility in comparison to germline testing. In order to assess utility of BRCA1/2 somatic mutation testing over and above 
germline testing, we tested 18 FFPE tumor samples from patients with ovarian cancer to estimate the prevalence of low allele frequency, actionable, somatic variants in a small cohort. We detected somatic variants of significance in BRCA1/2 and other homologous recombination genes which were actionable and would not have been detected through germline testing. Our results were consistent with other published reports, confirming that somatic BRCA1/2 testing in patients with advanced-stage epithelial ovarian cancer may provide clinicians and patients with additional information that could influence clinical management.

\section{WITHDRAWN}

\section{DETERMINATION OF CLINICAL APPLICABILITY AND DEMONSTRATION OF CONCORDANCE OF NGS TO INVESTIGATE AML}

Webb $\mathrm{F}^{\mathrm{I}}$

${ }^{I}$ Cytogenetics, ACT Pathology, Garran, Australia

${ }^{2}$ Haematology, ACT Pathology, Garran, Australia

${ }^{3}$ The Australian National University, Acton, Australia

Background: The revised WHO (2017) classification refers to sequence variants in AML for diagnosis, prognosis, therapy decisions and monitoring. We assessed the clinical applicability of next generation sequencing (NGS) in our center and tested concordance of sequence variants with previous results from other centers. Method: DNA was extracted and library preparation of DNA was performed as per the Illlumina TruSight Myeloid method (54 gene panel) from cell pellets of 24 patients with AML whose samples were archived in the ACT Haematology Research Tissue Bank after consent. Pooled library preparations were run on the MiSeq Next Generation Sequencer and data were analyzed using Illumina's BaseSpace software. Data were compared with previous results obtained from other laboratories. Results: Full concordance between NGS and previous results was observed for FLT3 TK2, NPMI, CEBPA, and CALR. As expected, the FLT3 ITD was not able to be to be detected by NGS. Sample processing including DNA extraction, library preparation, run time on equipment, data analysis and reporting is able to be performed in one week. Conclusion: NGS results were concordant for all variants (excluding FLT3 ITD mutations) that had previously been detected. Moreover, this panel approach to testing demonstrates clinical utility in the timely provision of variant information for diagnosis and improved subclassification of AML.

\section{POLYGENIC BREAST CANCER RISK: A PROSPECTIVE STUDY OF UPTAKE AND OUTCOMES AMONG HIGH-RISK WOMEN}

Yanes $T^{1,2}$, Meiser $B^{1}$, Kaur $R^{1}$, Scheepers-Joynt $M^{3}$, Young $M^{3,4}$, Barlow-Stweart $K^{5}$, John $T^{6}$, Harris $M^{7}$, Antill $Y^{8}$, Burke ${ }^{9}$, Roscioli $T^{10}$, Halliday J ${ }^{11,12}$, Mitchell $P^{2}$, James $P^{3,13}$

${ }^{1}$ Prince of Wales Clinical School, Faculty of Medicine, UNSW Sydney, Sydney, Australia

${ }^{2}$ School of Psychiatry, Faculty of Medicine, UNSW Sydney, Sydney, Australia

${ }^{3}$ Parkville Familial Cancer Centre, Peter MacCallum Cancer Centre and the Royal Melbourne Hospital, Melbourne, Australia

${ }^{4}$ Garvan Institute of Medical Research, Darlinghurst, Australia

${ }_{5}$ Sydney Medical School Northern, University of Sydney, Sydney, Australia

${ }^{6}$ Clinical Genetics Service, Austin Hospital, Melbourne, Australia

${ }^{7}$ Family Cancer Clinic, Monash Medical Centre, Melbourne, Australia

${ }^{8}$ Family Cancer Clinic, Cabrini Health, Melbourne, Australia

${ }^{9}$ Tasmanian Clinical Genetics Service, Royal Hobart Hospital, Hobart, Australia

${ }^{10}$ Department of Medical Genetics, Sydney Children's Hospital, Randwick, Australia

${ }^{11}$ Public Health Genetics, Murdoch Children's Research Institute, Melbourne, Australia

${ }^{12}$ Department of Paediatrics, University of Melbourne, Parkville, Australia

${ }^{13}$ Sir Peter MacCallum Department of Oncology, University of Melbourne, Melbourne, Australia

Background: Despite increasing scientific evidence regarding the utility of polygenic risk score (PRS) for families at high-risk of breast cancer, research findings are yet to be integrated into clinical practice. Before integrating polygenic information into clinical practice, it is important to understand the psychological implications. Aim: This prospective study aims to assess uptake of breast cancer PRS and ascertain the psychosocial and behavioral implications of receiving this information. Methods: Eligible women are invited to participate and receive their breast cancer PRS. Eligibility: affected and unaffected women currently enrolled in the Variants in Practice Study, who have a high or low PRS, and a personal and/or family history of breast cancer where genetic testing for BRCA1/2 is negative. Participants complete three self-administered questionnaires: T1 prior to result, T2 two weeks and T3 one year post receipt of PRS. Results: As of April 2018, 147/173 (85\%) participants reported interest in receiving their PRS with 25/173 (14\%) declining. Logistic regression showed that uptake of PRS was associated with having daughters $(O R=4.43,95 \%$ CI $[1.36,14.43], p=.013)$, higher uncertainty avoidance $(O R=0.6 .30,95 \%$ CI $[2.65,14.91]$, $p \leq .000)$ and higher response cost $(O R=.85$ (95\% CI [0.72, 0.99], $p=.043)$. Additional preliminary analysis showed a significant reduction in perceived risk (mean difference $-0.20, p=.013$ ), and general anxiety and depression (mean difference $-1.56, p=.002$ ) between T1 and T2. Conclusion: There is strong interest in receiving PRS among women at high-risk of breast cancer. Recruitment is ongoing, with additional data regarding short-term and longterm psychological and behavioral impact of receiving PRS to be collected.

\section{CLINICAL GENETICS}

\section{FACEMATCH: A SECURE PORTAL PROVIDING AUTOMATED FACE-MATCHING FOR INDIVIDUALS WITH UNDIAGNOSED INTELLECTUAL DISABILITY}

Dudding-Byth $T^{1,2,3}$, Boyle J', Riveros $C^{2}$, Attia $J^{2,4}$, Hackett $A^{1,5}, 0$ 'Donnell $S^{5}$,

$\overline{\text { Baxter } A^{5} \text {, Field }} M^{1}$, Kamien $B$, Roberts $]^{2}$, Cowling $S^{2}$, Brain $S^{7}$, Lovell $B^{7,8}$

${ }^{1}$ Hunter New England Health Service, Newcastle, Australia

${ }^{2}$ Hunter Medical Research Institute, Newcastle, Australia

${ }^{3}$ University of Newcastle GrowUpWell Priority Research Centre, Newcastle, Australia

${ }^{4}$ University of Newcastle, Newcastle, Australia

${ }^{5}$ Hunter Genetics, Waratah, Newcastle, Australia

${ }^{6}$ Genetic Services of Western Australia, Subiaco, Australia

${ }^{7}$ Imagus Technology, Brisbane, Australia

${ }^{8}$ University of Queensland, Brisbane, Australia

Despite advances in genomic sequencing, $60-70 \%$ of individuals with moderate to severe intellectual disability [ID] remain 
undiagnosed. Many individuals with ID have facial features which can provide a clue to diagnosis, and the discovery of novel ID genes typically requires confirmation of candidate gene variants in multiple unrelated individuals with a similar phenotype. Historically, clinical geneticists manually presented images of undiagnosed patients to their colleagues. The matchmaker exchange initiative now enables sharing of Human Phenome Ontology [HPO] terms and relevant candidate gene information, but there is no ability to match on facial images. Our aim was to establish an international portal providing automated face-matching for individuals with undiagnosed moderate to severe ID. FaceMatch design and development was undertaken by our multidisciplinary team with parent and doctor engagement. The parent empowering model offers dual parent/ doctor participation which can be initiated by a parent or a doctor. The secure child profile page contains images, medical history, and genetic information. Consent and email notifications are within the system. Using advanced IMAGUS face recognition technology, FaceMatch compares every new image automatically against images within the facebase, generating a matching score and ranking list. The FaceMatch team notify participating doctors of high probability facial matches. Facematch is also a member of the international Minerva Phenotyping Consortium. Conclusion: FaceMatch (https://facematch.org.au/) is the first of its kind to provide automated face-matching for individuals who remain undiagnosed following exome or genome sequencing. Ongoing research includes the incorporation of HPO terms into the matching algorithm.

\section{EXPLORING NON-CODING VARIATION IN LARGE $X$-LINKED PEDIGREES}

\author{
Field $M^{\prime}$, Hackett $A^{\prime}$, Ivancevic $A^{2}$, Kayumi $S^{2}$, Dudding-Bligh $T^{\prime}$, Palmer $E^{\prime}$, \\ Christie $L^{\prime}$, Sharma $R^{2}$, Gecz J,3, Corbett $M^{2}$ \\ ${ }^{I}$ Genetics Of Learning Disability Service, Newcastle, Australia \\ ${ }^{2}$ Robinson Research Institute and Adelaide Medical School, Adelaide, Australia \\ ${ }^{3}$ SAHMRI, Adelaide, Australia
}

Over the last 15 years, great advances have been made in our understanding of the genetics of X-linked intellectual disability. Our focus on variants in coding exons has left us with a unique group of unresolved families that are likely to be highly enriched for causative non-coding variants. We will discuss four large $\mathrm{X}$-linked pedigrees where we identified functionally distinct non-coding mutations in each case. Initially we have focused on families with either distinctive phenotypes or significant linkage data that can be used to reduce the search field. This experience is helping us to develop a diagnostic pipeline using integrated analysis of both whole genome (WGS) and RNA-Seq data to simultaneously identify and evaluate potentially relevant non-coding variants. We will describe one case where a rare coding variant in a known XLID gene within the linkage interval might have been reported as pathogenic. Careful consideration of the phenotype prompted further analysis of WGS and RNA-Seq data and identified a non-coding variant with a damaging functional effect on expression of a gene more closely correlated to the phenotype. This instructive case illustrates that close interaction between laboratory services and clinician are vital to achieve an accurate diagnosis for families. We suggest that large unresolved X-linked families may prove a great training ground to explore non-coding variation as they did for our early understanding of the genetics of intellectual disability.

\section{SEXUAL DISCORDANCE IN MONOZYGOTIC TWINS - HOW?}

Gear $\mathrm{R}^{\prime}$, Beddow $\mathrm{A}^{2}$, Poke $\mathrm{G}^{\mathrm{I}}$

${ }^{1}$ Genetics Health Service NZ, Central Hub, New Zealand

${ }^{2}$ Wellington Regional Genetics Laboratory, New Zealand

Case Report: A 32-year-old female underwent a single embryo in vitro fertilization transfer, after bilateral salpingectomies for two previous ectopic pregnancies. At 20 weeks gestation, an ultrasound revealed a mono-chorionic, di-amniotic twin pregnancy, with apparent sex discordance. The twins were delivered in good condition, without any physical anomalies or dysmorphic characteristics, but on examination twin 1 had normal female external genitalia and twin 2 had normal male external genitalia. What is the explanation? A microarray on a blood sample from twin 1 showed a 46XY molecular karyotype. QF-PCR was done on buccal swabs from both twins, which confirmed them to be monozygotic. The same buccal swab from twin 1, showed her to be 45X/46XY mosaic, which was also confirmed on skin biopsy. An ultrasound of the abdomen revealed a uterus and streak gonads. On this basis, twin 1 was deemed to have Turner syndrome variant, presumably secondary to an early postzygotic loss of the $\mathrm{Y}$ chromosome in the cell line, leading to her gonadal development and secondary female sexual characteristics.

\section{QUALITY OF LIFE IN HEREDITARY HEMORRHAGIC TELANGIECTASIA - A SINGLE CENTER AUSTRALIAN STUDY}

Healy $L^{1,2}$, Winship I',2

${ }^{I}$ Royal Melbourne Hospital, Parkville, Australia

${ }^{2}$ University of Melbourne, Parkville, Australia

Hereditary hemorrhagic telangiectasia (HHT) is characterized by telangiectasiae and arteriovenous malformations. Epistaxis is common and often severe, and internal bleeding also occurs. Aim: To assess HHT impact on Quality of Life (Qol). Methods: After protocol approval, standardized questionnaires [EuroQol EQ-5D-3L, Australian AQol6D and Epistaxis Severity Score (ESS)] were administered, and scores compared with published normative data. Study use of AQo16D and EQ5D was registered, and Qol parameters were compared to normative data (two-tailed $t$ test). Results: 39 patients with HHT were invited to participate. 16 gave written informed consent and 13 participated (age 44.2 $\pm 14.4,5$ males), 13 completing ESS and AQol6D, and 12 also completing EQ5D. Mean ESS was $5.03 \pm 2.58 \mathrm{SD}$ (median 5.25, range $0-8.19$ ). In only 3 was ESS score $<3$. All required supplemental iron. Mean $\pm S D$ EQol Visual Analogue Scale score was $72.8 \pm 14.2$ (95\% CI [64.4, 81.2]), significantly below published norms for age 18-79 in New Zealand (81.8), Argentina (90.2), Germany (93.8), UK (85.6) and US (86.7). EQVAS norms significantly reduce with age. No population norms are available for Australia. Six noted moderate pain/discomfort, 5 difficulty with usual activities ( 4 moderate, 1 extreme), and 4 moderate anxiety/depression. AQol6D scores in 4/6 dimensions were reduced, maximally for pain $(52.3 \pm 9.3$ vs. $86.4 \pm 0.19, t=141.4$, $p<.0001)$. Total score was also lower: $69.8 \pm 11.2$ vs. $84.4 \pm 16.4$, $(t=3.2025, p=.0014)$. 'Relationships' and 'Mental Health' scores were normal, and age did not correlate with AQol6D scores. Patients with regular nosebleeds noted that health disrupted usual activities including work. Conclusion: HHT significantly impacts on QoL in our patients.

\section{ABSENCE OF RENAL PHENOTYPE IN HEREDITARY HEMORRHAGIC TELANGIECTASIA}

Healy $L^{1,2}$, Nicholls $K^{1,2}$, Gibson $R^{1,2}$, Stella $D^{1,2}$, Bogwitz $M^{\prime}$, Taylor J', Walsh $M^{\prime}$, Donaldson L', Winship I,2

${ }^{1}$ Royal Melbourne Hospital, Parkville, Australia

${ }^{2}$ The University of Melbourne, Parkville, Australia

The autosomal dominant disorder hereditary hemorrhagic telangiectasia (HHT) is characterized clinically by mucocutaneous telangiectasia and internal arteriovenous malformations (AVMs), pathologically by dysregulated angiogenesis, and genetically most commonly by a mutation in Endoglin (ENG) and Activin receptorlike kinase 1 (ACVRL1). As a highly vascular organ, the kidney might be expected to be affected, but little information is available. We assessed renal structure and function in HHT patients with a 
confirmed mutation in the ENG or ACVRL1, known AVMs and significant epistaxis. Methods: Patients gave informed consent to an HREC-approved protocol including targeted renal ultrasound and MRI, urinary cell counts, albuminuria, serum creatinine $(\mathrm{SeCr})$ and urea. The standard Modification of Diet in Renal Disease (MDRD) algorithm was used to estimate glomerular filtration rate (eGFR).7 Results: Eleven patients (5 male), aged $43 \pm 14.1$ years, mean Epistaxis Severity Score 5.0 \pm 2.6 , completed renal function testing. Ten underwent ultrasound, and 9 MRI in addition. Seven had mutations in ACVRL1, 4 in ENG. Six had internal organ AVMs, 4 previously undergoing invasive management. All required supplemental iron. Renal function was normal in all patients, mean $\mathrm{SeCr} 70.7 \pm 15.8$ $\mu \mathrm{mol} / \mathrm{L}$ and eGFR normal. No patient had abnormal albuminuria, proteinuria, urinary RBC or WBC. MRI and ultrasound examinations were normal except for a small simple cyst in each kidney in one 49-year-old woman. A 12th patient, a 72-year-old man, was withdrawn from the study after renal carcinoma was found on ultrasound scan. Conclusion: Despite the vascularity of the kidneys, we found no evidence of renal involvement. This observation warrants further consideration.

\section{INTRANASAL BEVACIZUMAB TO REDUCE EPISTAXIS IN HEREDITARY HEMORRHAGIC TELANGIECTASIA - A PILOT STUDY}

Healy $L^{1,2}$, Rimmer $J^{1,3}$, Hong $E^{\prime}$, Nicholls $K^{1,2}$, Bogwitz $M^{\prime}$, Taylor $J^{\prime}$, Walsh $M^{\prime}$, Winship I, $^{1,2}$

${ }^{1}$ Royal Melbourne Hospital, Parkville, Australia

${ }^{2}$ University of Melbourne, Parkville, Australia

${ }^{3}$ Monash University, Clayton, Australia

The autosomal dominant disorder hereditary hemorrhagic telangiectasia (HHT) is characterized clinically by mucocutaneous telangiectasia and internal arteriovenous malformations(AVMs), pathologically by dysregulated angiogenesis, and genetically most commonly by a mutation in Endoglin(ENG) or Activin receptor-like kinase 1(ACVRL1). The most common symptom experienced by patients is severe and recurrent epistaxis. The Epistaxis Severity Score (ESS) is a standardized measure of the impact of nosebleeding. Apart from discomfort, frequent blood loss may induce anemia. We report the topical use of a monoclonal antibody to VEGF, Bevacizumab, aiming to limit blood vessel formation in the nose. Methods: Patients with a confirmed genetic diagnosis of HHT and ESS $>3$ (accepted threshold of severe epistaxis) gave informed consent to an HREC-approved protocol. Regular phone surveys of participants' ESS were performed. After 3 months, Bevacizumab $(10 \mathrm{mg} / \mathrm{ml})$ was administered by metered nasal spray, 30 mins after nasal rinsing (FLO Sinus Care kit, ENT Technologies Australia). Dose was $0.1 \mathrm{ml} / \mathrm{spray}$, twice daily into each nostril for 10 days. ESS was calculated at time points: -3 months, baseline, 1 week, 2 weeks, 1 month, 2 months, 3 months. Results were analyzed by 1-way ANOVA. Results: Six patients ( 3 male) participated. The ESS values at -3 months $($ mean $+\mathrm{SD}=6.320 \pm 1.37)$ and baseline $(5.980 \pm 1.48)$ were not significantly different, however ESS at all subsequent time points, post treatment, was significantly reduced $(p<.001)$. Five of the six patients fell below an ESS of 3 after seven days of using the spray. Conclusion: In combination with nasal rinsing, intranasal Bevacizumab may be an effective therapy for epistaxis in HHT patients.

\section{FURTHER DELINEATION OF THE BAINBRIDGE-ROPERS} SYNDROME PHENOTYPE IN AUSTRALIAN PATIENTS

\footnotetext{
Higgins $M^{\prime}$, McGaughran J', White $S^{2}$, Yeung $A^{2}$

${ }^{I}$ Genetic Health Queensland, Brisbane, Australia

${ }_{2}$ Victorian Clinical Genetics Services, Melbourne, Australia
}

Background: Bainbridge-Ropers syndrome (BRPS), caused by ASXL3 heterozygous loss of function mutations, is a newly de- scribed intellectual disability syndrome. To date, less than 30 individuals have been reported in the literature. Concordant clinical features include developmental delay, absent speech, muscle hypotonia, feeding difficulties and a distinctive craniofacial phenotype. In this report, we further delineate the BRPS phenotype by describing a cohort of Australian patients not previously reported. Methods: Next generation sequencing was performed in all patients included in this study, which found de novo variants in ASXL3. Detailed clinical information and photographs were collected as part of this study. Consent was obtained from the patients' families. Results: Summaries of patients' clinical features, photos and ASXL3 mutations will be reported here. This section will also include a comparison of our patients to patients previously reported in the literature. Discussion: This case series expands the BRPS phenotype with newly reported features.

\section{IDENTIFICATION AND CHARACTERIZATION OF GENES RELATED TO DEVELOPMENTAL DYSPLASIA OF THE HIP}

Ibrahim $\mathrm{T}^{\text {I }}$, Hegazy A, AINouri M, Robay A, Rodriguez-Flores J, Crystal R, Greenblatt $M$

${ }^{I}$ Sidra Medical and Research Center, Qatar

Background: Developmental dysplasia of hip (DDH) is a common congenital hip dislocation disorder, if not recognized and managed appropriately, can lead to permanent disability. Though there is evidence for a genetic cause for many cases of DDH, the genetic basis of DDH is largely unknown. Methods: Ten families displaying multiple family members with DDH underwent clinical characterization, and genetic variants displaying linkage with the DDH phenotype were determined by whole exome sequencing. Tendons from TNXB-/- mice were examined using transmission electron microscopy (TEM). Results: A Qatari family with two children affected by DDH where variants in two genes associated with forms of Ehlers Danlos Syndrome, TNXB and COL5A2, were found to display linkage with $\mathrm{DDH}$. These variants are present in approximately $2 \%$ of Qataris and rare in other populations. TEM on patellar tendon of TNXB-/- mice demonstrated marked abnormalities in collagen fibril organization. Discussion/Conclusion: These findings raise the possibility that some cases of DDH can be considered an attenuated form of the joint laxity phenotype seen in Ehlers Danlos Syndrome. Studies in TNXB-/- mice suggest that mutations in TNXB are expected to alter the biomechanical properties of tendon and predispose to DDH though a connective tissue laxity effect. Consideration of whether other Ehlers Danlos-associated phenotypes are present in Qatari DDH patients may be warranted. Given the high frequency of these alleles in Qataris, these variants may represent a common Qatari-specific cause of DDH. Based on these findings, we are studying the presence of these variants in other families with DDH.

\section{AN INNOVATIVE MODEL OF SERVICE DELIVERY ADAPTING TO THE CHANGING LANDSCAPE OF CLINICAL GENETICS}

Kearton S', Freeman L', Department of Clinical Genetics Team '

${ }^{I}$ Royal North Shore Hospital, St Leonards, Australia

As with any clinical discipline that is changing, there is a need to assess the adequacy of current service arrangements to determine whether services are meeting the health needs of patients safely, equitably and ethically. The Clinical Genetics Department at Royal North Shore Hospital is a busy metropolitan unit providing diagnosis, counseling and management advice to individuals and their families who have, or are at risk of having a genetic condition in the prenatal, pediatric and adult setting. Services are delivered by a team of clinical geneticists, genetic fellows and genetic counselors. Over the past few years, a number of informal service models have 
evolved within our service to cope with increasing demands on genetic services. Our Model of Service Delivery is centred on triaging referrals into a clinician-led clinic or a genetic counselor-led clinic based on the needs of the patient. The model aims to be patient centred while ensuring each team member's skill set is best utilized. We hypothesise that our Model of Service Delivery optimises healthcare delivery and efficiency as a viable option as we progress to mainstreaming genetics and working with non-genetics specialists. We also hypothesize that the Model increases job satisfaction and provides opportunity to specialize in particular areas of interest for geneticists and counselors alike. We will present further details of our Model of Service Delivery, including a comparison with similar large metropolitan services, and the outcomes of a focus group held within the department, which considered the benefits and limitations of the model.

\section{PRE-GENETIC CLINIC RESOURCE EVALUATION FOR ADULTS WITH INTELLECTUAL DISABILITY: THE PRE-GENETIC CLINIC AID}

\footnotetext{
Kotwal $\mathrm{H}^{\prime}$, Fleming $\mathrm{J}^{\prime}$, Barlow-Stewart $\mathrm{K}^{\prime}$, Leffler $\mathrm{M}^{2}$, Murray $\mathrm{L}^{2}$, Silberbauer $\mathrm{L}^{3}$, Boyle $\mathrm{J}^{2}$, Palmer $\mathrm{E}^{2,4}$

${ }^{1}$ Discipline of Genetic Medicine, University of Sydney Medical School - Northern, St. Leonards, Sydney, Australia

${ }_{2}^{2}$ Genetics of Learning Disability, Waratah, Hunter New England, Australia

${ }^{3}$ Nepean Genetics, Nepean, Australia

${ }^{4}$ School of Women's and Children's Health, University of New South Wales, Sydney,

Australia
}

Among the several ways of facilitating inclusivity in their healthcare, people with intellectual disability (PWID) consistently identify the importance of making information accessible (Easy Read) and relevant. Currently no resources aim at supporting PWID attendance at clinical genetics appointments; however, evidence suggests such appointments can provoke confusion and anxiety for PWID. Our research question was whether we could develop an accessible, relevant and appropriate booklet for PWID to read with their carers prior to attending clinical genetics services. With input from Easy Read experts and members of the NSW Council for Intellectual Disability, we developed the information booklet 'Getting ready for your visit to the genetic clinic'. To assess its acceptability, an online/hard copy anonymous survey is being used. The sample cohort is approximately 50 health care professionals (HCP) and carers of PWID familiar with genetics services. Recruitment is via affiliated clinical services, the list-serve of clinical genetics organizations and genetic support groups. Using descriptive statistics, questions will collect information on perceived relevance and readability of the booklet content and its utility for HCP and carers. The results will inform improvement and refinement of the booklet; to be evaluated further by PWID (intended end-users), prior to becoming freely available online. The outcome of this study will be a customisable booklet transferrable across national genetic services. We envisage this booklet can be utilized as a guide for development of other clinical genetic resources for PWID, aiming to reducing health care inequities, improving client satisfaction and involving PWID in their healthcare decisions.

\section{HOW WELL DOES THE HUMAN PHENOTYPE ONTOLOGY DESCRIBE SKELETAL DYSPLASIAS?}

Lau $\mathrm{M}^{1,2}$, Zankl A $\mathrm{A}^{2,3,4,5}$

${ }^{I}$ School of Medicine, University Of New South Wales, Sydney, Australia

${ }^{2}$ Garvan Institute of Medical Research, Sydney, Australia

3 Department of Clinical Genetics, The Children's Hospital at Westmead, Westmead, Australia

${ }^{4}$ Sydney Medical School, The University of Sydney, Sydney, Australia

${ }^{5}$ Kinghorn Centre for Clinical Genomics, Sydney, Australia

The Human Phenotype Ontology (HPO) is a controlled vocabulary of well-defined terms to systematically describe the clinical features of rare genetic disorders. The HPO Consortium also annotates rare disorders with HPO terms that phenotypically characterise the disease. The HPO has enabled the computational processing of phenotype data and has quickly become an integral part of many clinical genomics applications. However, how well the over 11,000 terms and over 100,000 disease annotations cover a clinical area of interest has not been systematically studied. Here, we explore the suitability of HPO to capture the phenotypic diversity of skeletal dysplasias, a group of rare genetic disorders affecting skeletal development. We extracted 5,477 phenotypic descriptions of 223 disorders from a classic textbook and tried to match them to HPO terms through a combination of text mining and manual curation. We also compared 6,351 disease annotations from the HPO consortium against the phenotype descriptions extracted from the textbook. At least $20 \%$ of textual descriptions could not be matched with a HPO term, indicating that the HPO has gaps in its skeletal dysplasia terminology. Less than $40 \%$ of the disease annotations from the HPO Consortium could be matched against textual descriptions in the textbook, indicating major discrepancies in how skeletal dysplasias are characterized by the HPO as compared to a classic textbook. The HPO's ability to describe the skeletal dysplasia domain is currently limited. Manual creation may be necessary to identify missing terms and refine annotations.

\section{VELAGLUCERASE ALFA CLINICAL TRIAL PATIENTS: AN ANALYSIS FROM THE GAUCHER OUTCOME SURVEY}

Lau $\mathrm{H}^{1}$, Belmatoug $\mathrm{N}^{2}$, Botha ${ }^{3}$, Giraldo $\mathrm{P}^{4,5}$, Hughes $\mathrm{D}^{6}$, Panahloo $\mathrm{Z}^{3}$, Zimran $\mathrm{A}^{7}$

${ }^{I}$ New York University, Langone Medical Center, New York, USA

${ }^{2}$ Hôpitaux Universitaires Paris Nord Val de Seine, Clichy, France

${ }^{3}$ Shire, Zug, Switzerland

${ }^{4}$ CIBER de Enfermedades Raras, IIS Aragon, Zaragoza, Spain

${ }^{5}$ Translational Research Unit, IIS Aragon, Zaragoza, Spain

${ }^{6}$ University College Medical School, London, UK

${ }^{7}$ Shaare Zedek Medical Center and the Hebrew University-Hadassah Medical School, Jerusalem, Israel

Gaucher disease (GD) is a rare, autosomal recessive condition, characterized by hepatosplenomegaly, thrombocytopenia and anemia. The benefits of treatment are well documented within the clinical trial environment; however, data on whether initial clinical trial improvements are maintained as patients transition into real-life clinical practice are limited. This evaluation included 65 patients who participated in velaglucerase alfa clinical trials (TKT025, TKT025 EXT, TKT032, TKT034, HGT-GCB-039 or open label extension HGT-GCB-044) and then enrolled into the Gaucher Outcome Survey (GOS), an international registry for patients with confirmed GD regardless of type or treatment status, as part of their routine clinical care. Parameters assessed included hemoglobin levels, platelet counts, liver and spleen volume, chitotriosidase and CCL18 levels. At clinical trial entry, $84.6 \%$ patients were aged $\geq 18$ years, $52.3 \%$ were female, $87.3 \%$ had an N370S-containing genotype, $84.6 \%$ had an intact spleen or partial splenectomy, $56.9 \%$ were treatment naïve and $43.1 \%$ had previously received imiglucerase ('switched patients'). Treatment naïve patients showed improvements in all measured clinical parameters during the clinical trial period (mean 58.8 months) that were maintained during the GOS period (mean 59.7 months). Normalization of some clinical parameters was maintained for up to 84 months. For switched patients, improvements achieved on previous therapy were maintained throughout both clinical trial and GOS periods. Similar findings were achieved for 29 patients ( 28 treatment naïve, 1 switched) who received only velaglucerase alfa and had available laboratory values. This analysis suggests that improvements in clinical parameters achieved during clinical trials are maintained long-term in real-life clinical practice. 


\section{MANAGEMENT GOALS AND NORMALIZATION CONCEPT FOR GAUCHER DISEASE: RESULTS FROM A SURVEY OF EXPERT PHYSICIANS}

\author{
Mehta $A^{\prime}$, Panahloo $Z^{2}$, Di Rocco $M^{3}$, Goker-Alpan $O^{4}$, Hughes $D^{\prime}$, \\ Kindmark $A^{5}$, Kuter $D^{6}$, Lukina $E^{7}$, Nakamura $K^{8}$, Oliveri $B^{9}$, Perez-Lopez J ${ }^{10}$, \\ Schwartz I $I^{11,12}$, Serratrice $C^{13}$, Szer J ${ }^{14}$, Zimran A ${ }^{15}$, Pastores $G^{16}$ \\ ${ }^{I}$ Royal Free London NHS Foundation Trust, University College London, London, \\ United Kingdom \\ ${ }^{2}$ Shire, Zug, Switzerland \\ ${ }^{3}$ Unit of Rare Diseases, Department of Pediatrics, Giannina Gaslini Institute, Genoa, \\ Italy \\ ${ }^{4}$ Lysosomal Disorders Unit and Center for Clinical Trials, O\&O Alpan, LLC, Fairfax, \\ USA \\ ${ }^{5}$ Uppsala University Hospital, Uppsala, Sweden \\ ${ }^{6}$ Center for Hematology, Massachusetts General Hospital, Harvard Medical School, \\ Boston, USA \\ ${ }^{7}$ National Research Center for Hematology, Moscow, Russia \\ ${ }^{8}$ Kumamoto University Hospital, Kumamoto, Japan \\ ${ }^{9}$ Laboratorio de Osteoporosis y Enfermedades Metabolicas Oseas \\ INIGEM-UBA-CONICET, Buenos Aires, Argentina \\ ${ }^{10}$ Unit of Rare Diseases, Hospital Vall d'Hebron, Barcelona, Spain \\ ${ }^{11}$ Hospital de Clínicas de Porto Alegre, Porto Alegre, Brazil \\ ${ }^{12}$ Department of Genetics, Universidade Federal do Rio Grande do Sul, Porto Alegre, \\ Brazil \\ ${ }^{13}$ Geneva University Hospital, Thonex, Switzerland \\ ${ }_{14}$ Royal Melbourne Hospital, Parkville, Australia \\ ${ }^{15}$ Shaare Zedek Medical Center and the Hebrew University-Hadassah Medical School, \\ Jerusalem, Israel \\ ${ }^{16}$ University College Dublin, and Mater Misericordiae University Hospital, Dublin, \\ Ireland
}

Gaucher disease is a rare, autosomal recessive condition caused by deficient activity of the lysosomal enzyme $\beta$-glucocerebrosidase, and characterized by hepatosplenomegaly, thrombocytopenia, and anemia. The availability of enzyme replacement therapy and substrate reduction therapy has resulted in improvements in the principal manifestations of the disease, and normalization of some modifiable disease parameters is now possible for certain patients. The primary objective of the survey was to explore the perspectives of global Gaucher disease experts on the concept of 'normalization' of modifiable disease parameters in the management of patients with Gaucher disease. The survey was carried out in May 2017 and included 16 global Gaucher disease experts from 14 countries. $56 \%$ of respondents indicated their agreement with aiming for normalization of modifiable disease parameters, with strongest agreement for spleen size ( $81 \%$ of participants), liver size $(75 \%)$, and hemoglobin levels (63\%). 56\% of respondents agreed on efforts to normalize platelet count and bone mineral density. Most respondents supported the use of all domains of the 36-item Short Form Health Survey in routine assessment, although the vitality, mental health, and social functioning domains were considered less important by one-third of respondents. Chitotriosidase, CCL18, and lyso-Gb1 (glucosylsphingosine) were considered the most useful biomarkers for monitoring Gaucher disease progression and response to treatment. In conclusion, respondents agreed that targeting the normalization of some (organ size) but not all disease parameters may be a beneficial target in the management of patients with Gaucher disease.

\section{PROPKD SCORE PREDICTS KIDNEY DECLINE AMONG AUSTRALIAN PATIENTS WITH AUTOSOMAL DOMINANT POLYCYSTIC KIDNEY DISEASE}

\footnotetext{
Chan $\mathrm{S}^{1,2}$, Patel $\mathrm{C}^{3}$, Mallett $\mathrm{A}^{1,2}$

${ }^{1}$ Kidney Health Service, Metro North Hospital and Health Service, Brisbane, Australia

${ }^{2}$ Faculty of Medicine, The University of Queensland, Brisbane, Australia

${ }^{3}$ Genetic Health Queensland, Royal Brisbane and Women's Hospital, Metro North

Hospital and Health Service, Brisbane, Australia
}

Background: The PROPKD score, developed from a single-country cohort study, enables risk stratification of progression to end-stage kidney disease in patients with Autosomal Dominant Polycystic Kidney Disease (ADPKD). The aim of this study was to validate whether the PROPKD score predicts kidney function decline in clin- ical practice among ADPKD patients. Methods: A cross-sectional study was conducted of 39 unrelated individuals with genetically confirmed ADPKD encountered by the Queensland Renal or Genetics Services between 1st January 1994 to 30th April 2018. Clinical and genetic factors were examined using the PROPKD score, to assess its relationship with kidney function decline prediction over a three year period. Results: Of the 39 individuals, 15 patients were low risk, 17 were intermediate risk and 7 were high risk. The median age for the commencing renal replacement therapy (RRT) was 60 years (low-risk group), 54.5 years (intermediate-risk group), and 55.3 years (high-risk group). Of those who commenced RRT, 1 was high risk, 6 were intermediate risk and 1 was low risk. One high risk patient underwent pre-empt live transplant at 48 years. The median change in estimated glomerular filtration rate (CKD-EPI) in the low risk group was $1.5 \mathrm{ml} / \mathrm{min} / 1.73 \mathrm{~m} 2 / \mathrm{yr}, 12.5 \mathrm{ml} / \mathrm{min} / 1.73 \mathrm{~m} 2 / \mathrm{yr}$ in the intermediate risk group and $15 \mathrm{ml} / \mathrm{min} / 1.73 \mathrm{~m} 2 / \mathrm{yr}$ in the high risk group $(p<.001)$. There were no deaths from this cohort. Conclusion: The PROPKD score accurately predicts kidney function decline in patients with ADPKD in this cohort. This prediction tool may enable future personalized clinical prognostication and therapeutic management of ADPKD.

\section{DEMOGRAPHIC CHARACTERISTICS AND GLA MUTATIONS IN TAIWANESE PATIENTS WITH DATA IN THE FABRY OUTCOME SURVEY}

Hsu $\mathrm{T}^{\prime}$, Yu W' $\mathrm{W}^{2}, \mathrm{Li} \mathrm{H} \mathrm{H}^{1}, \mathrm{Fu} \mathrm{Y^{3 }}$, Lin $\mathrm{C}^{4}$, Chiu $\mathrm{P}^{5}$, Gurevich $\mathrm{A}^{6}$, Botha $J^{6}$, Niu $\mathrm{D}^{1}$

${ }^{1}$ Department of Pediatrics, Taipei Veterans General Hospital, Taipei 112, Taiwan

${ }^{2}$ Division of Cardiology, Department of Medicine, Taipei Veterans General Hospital, Taipei 112, Taiwan

${ }^{3}$ Department of Pediatrics, China Medical University Children's Hospital, Taichung, Taiwan

${ }^{4}$ Division of Cardiology, Department of Internal Medicine, National Cheng Kung

${ }^{4}$ Division of Cardiology, Department
University Hospital, Tainan, Taiwan

${ }_{5}^{5}$ Department of Pediatrics, Kaohsiung Veterans General Hospital, Kaohsiung, Taiwan Shire, Zug, Switzerland

Fabry disease is caused by mutations to the $\alpha$-galactosidase $\mathrm{A}$ (GLA) gene, resulting in deficient $\alpha$-galactosidase A activity and accumulation of glycosphingolipids in the heart, kidney, skin and brain. Newborn screening programs have shown the incidence of Fabry mutations to be much higher in the Taiwanese male population ( 1 in $\sim 1,500)$ than in the general population ( 1 in $\sim 50,000$ males). As of August 2017, the Fabry Outcome Survey (FOS; NCT\# 03289065), a worldwide disease registry for patients with documented Fabry disease, included 262 Taiwanese patients (55\% female, median [range] age at FOS entry $52[0-85]$ years). The most prevalent GLA mutation was c.936+919G $>$ A (IVS4+919G $>$ A) ('IVS4'), present in 79\% (191/242) of patients with available data. Median (range) age at symptom onset was 52.0 (13-69) years for patients with the IVS4 mutation, compared with 12.0 (4-54) years for those with non-IVS4 mutations. This is consistent with the known association between IVS4 and later-onset disease. Overall, 110/262 patients $(42.0 \%)$ received enzyme replacement therapy (ERT), although there was a clear difference in the proportion of treated patients between those with and without the IVS4 mutation (63/191 [33.0\%] vs 47/71 [66.2\%], respectively). Among the 108 patients who received agalsidase alfa, those with IVS4 started ERT at a median (range) age of 60 (24-74) years, compared with 42 (3-79) years for those with non-IVS4 mutations. Among patients with IVS4 and non-IVS4 mutations, respectively, $6.1 \%$ and $5.0 \%$ had eGFR $<60$ $\mathrm{mL} / \mathrm{min} / 1.73 \mathrm{~m} 2$ and $44.4 \%$ and $31.6 \%$ had LVMI $\geq 50 \mathrm{~g} / \mathrm{m} 2.7$ at baseline. 


\section{MENARCHE, MENOPAUSE, AND PREGNANCY DATA IN UNTREATED/AGALSIDASE ALFA-TREATED FEMALES IN THE FABRY OUTCOME SURVEY}

\author{
Hughes $D^{1}$, Barba Romero $M^{2}$, Gurevich $A^{3}$, Engrand $P^{4}$, Giugliani $R^{5}$ \\ ${ }^{I}$ Royal Free London NHS Foundation Trust, University College of London, London, \\ United Kingdom \\ ${ }^{2}$ Albacete University Hospital, Castilla-La Mancha University, Albacete, Spain \\ 3 Shire, Zug, Switzerland \\ ${ }^{4}$ Cytel Inc., Geneva, Switzerland \\ 5 Medical Genetics Service HCPA, Department of Genetics UFRGS, and INAGEMP, \\ Porto Alegre, Brazil
}

The effects of Fabry disease on reproductive and pregnancy-related outcomes are not well defined. This post hoc analysis examined data from untreated and agalsidase alfa-treated female patients in the Fabry Outcome Survey registry. Mean $(S D)$ age at menarche was 12.8 (1.6) years in 74 treated patients who received agalsidase alfa at any time and $13.1(1.5)$ years in 57 untreated patients. Mean $(S D)$ age at menopause was 48.7 (7.2) years in 66 treated women and 49.0 (5.3) years in 18 untreated women. Normal outcomes occurred in $21 / 23(91.3 \%)$ pregnancies in 21 treated women and 72/75 (96.0\%) pregnancies in 52 never-treated women. Spontaneous abortion occurred in $0 / 23$ pregnancies in 21 treated women and 2/75 (2.7\%) pregnancies in 52 never-treated women. Mean $(S D)$ age at pregnancy end was 31.1 (5.7) years in treated women and 27.3 (5.6) years in never-treated women. Mean $(S D)$ infant birth weights were 3,021 (689) $\mathrm{g}$ in 10 pregnancies in treated women and 3,194 (543) $\mathrm{g}$ in 38 pregnancies in never-treated women. Among treated and nevertreated women, respectively, mean $(S D)$ changes from before to after pregnancy were not significantly different for: estimated glomerular filtration rate, $-1.5(11.7)$ and $-3.1(13.6) \mathrm{mL} / \mathrm{min} / 1.73 \mathrm{~m} 2$; mean urinary protein, $-123.7(355.6)$ and $16.7(51.4) \mathrm{mg} / 24 \mathrm{~h}$; left ventricular mass index, -6.5 (15.6) and $-0.2(7.8) \mathrm{g} / \mathrm{m} 2.7$; Brief Pain Inventory (BPI) pain severity score, $0.0(1.8)$ and $0.6(2.2)$; and BPI interference score, 0.4 (2.1) and 0.6 (2.5). Regardless of treatment status, no significant changes in these renal and cardiac parameters were seen after pregnancy.

\section{SPECTRUM OF GENETIC MUTATIONS IN PATIENTS IN THE FABRY OUTCOME SURVEY}

Giugliani $R^{1}$, Beck $M^{2}$, Botha $\rfloor^{3}$, Feriozzi $S^{4}$, Gurevich $A^{3}$, Hughes $D^{5}$, Kampmann $\mathrm{C}^{6}$, Kemenyash $\mathrm{N}^{3}$, Nicholls $\mathrm{K}^{7}$, Niu D ${ }^{8}$, Pintos-Morell $\mathrm{G}^{9}$, Ramaswami $\mathrm{U}^{5}$, Reisin $\mathrm{C}^{6}$, Kemenyash $\mathrm{N}^{3}$, Nicholls $\mathrm{K}^{7}$, Niu $\mathrm{D}^{8}, \mathrm{P}$
$\mathrm{R}^{10}$, Sunder-Plassmann $\mathrm{G}^{11}$, West $\mathrm{M}^{12}$

${ }^{1}$ Medical Genetics Service HCPA, Department of Genetics UFRGS, and INAGEMP, Porto Alegre, Brazil

${ }^{2}$ Center for Pediatric and Adolescent Medicine, University Medical Center, Johannes Gutenberg University, Mainz, Germany

${ }^{3}$ Shire, Zug, Switzerland

${ }^{4}$ Belcolle Hospital, Viterbo, Italy

${ }^{5}$ Royal Free London NHS Foundation Trust, University College of London. London, UK

${ }^{6}$ Department of Pediatric Cardiology and Congenital Heart Disease, University Medical Center, University of Mainz, Department of Pediatrics, Mainz, Germany

${ }^{7}$ Royal Melbourne Hospital, Parkeville, Australia

${ }^{8}$ Taipei Veterans General Hospital, Institute of Clinical medicine, National Yang-Ming University, Taipei, Taiwan

${ }^{9}$ Rare Diseases Centre, Vall d'Hebron University Hospital, Badalona, Universitat

Autònoma de Barcelona, Spain

${ }^{10}$ Servicio de Neurología, Hospital Británico, Buenos Aires, Argentina

11 Division of Nephrology and Dialysis, Department of Medicine III, Medical

University of Vienna, Vienna, Austria

${ }_{12}$ Department of Medicine, Dalhousie University, Halifax, Canada

Fabry disease is associated with $>770$ mutations in the alphagalactosidase A (GLA) gene that influence the variable severity of the disease and are described as classical, later onset, or genetic variants of unknown significance (GVUS). There is growing interest in genotype-phenotype correlations in Fabry disease. The Fabry Outcome Survey (FOS; NCT\# 03289065; sponsored by Shire) is a worldwide disease registry for patients with a documented Fabry disease diagnosis. As of October 2017, FOS included 3437 patients, 1249 with signed informed consents to allow publication of genetic information. Of 1142 patients with available genetic information;
$623(54.6 \%)$ were receiving any enzyme replacement or chaperone therapy, and $689(60.3 \%)$ were female. Median (range) age at symptom onset was $14(0-71)$, with 6 patients having a family history of Fabry disease and age at symptom onset recorded in FOS as zero (ie, birth or $<1$ year). Median (range) age at diagnosis and treatment initiation was $35(0-85)$ and $44(4-81)$ years, respectively. Percentages of the 12 most prevalent GLA mutations were: classical, A143P (5.1\%), R227X (1.6\%), R301X (1.4\%), R227Q (1.1\%), R220X (1.1\%); later onset, IVS4 (18.8\%), N215S (8.9\%), R301Q (1.5\%), R112C (1.3\%); and GVUS, A143T (3.8\%), R118C (1.1\%), D313Y (1.1\%). Furthermore, $11.9 \%$ had other poorly defined mutations, primarily deletions $(6.9 \%)$. There was substantial geographic variability in prevalence of particular mutations (IVS4 in Taiwan, A143P in Canada, N215S in the UK). This genetic overview shows the prevalence of the most common mutations in FOS patients and should facilitate further exploration of genotype-phenotype correlations.

\section{DEVELOPING STRONG CLINICAL GOVERNANCE AT A CLINICAL GENETICS SERVICE}

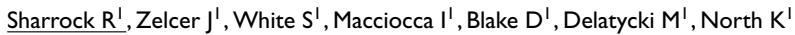
${ }^{I}$ Victorian Clinical Genetics Service, Melbourne/Parkville, Australia

Background: Demand for genetics and genomics services is growing in scope and complexity. It is important that clinical staff are supported by robust systems to ensure consistently safe, high quality care. In 2015-16 in Victoria there was a renewed focus on clinical governance across the state. In this context the Victorian Clinical Genetics Service (VCGS) Board commissioned a governance review to ensure high standards of governance are applied to the clinical service. The review, using criteria derived from the National Safety and Quality Health Service Standards (NSQHS) Standards, noted that VCGS has a number of clinical governance related process in place; however, these could be further developed and integrated, including a higher degree of consumer engagement in line with the NSQHS standards and contemporary community expectations. Aim: To ensure the VCGS meets and/or exceeds current clinical governance standards. Method: An analysis of VCGS processes was benchmarked against the National Standards to inform a priority work plan for system improvement. The VCGS is drawing on expertise through collaboration with campus partner The Royal Children's Hospital to ensure it meets and/or exceeds the National Standards. Results/Initial impressions: Clinical governance is underpinned by continuous improvement. The analysis to date identified the requirement to: review committee structures and governance framework; improve accessibility of policies and procedures; review compliance with legislation and regulations; implement an incident management system including data reporting; review how we partner with patients in their own care; establish systems for ongoing review, evaluation and continual improvement, including the governance framework itself.

\section{THE TELEPHONE RESULTS CLINIC: ACCOUNTABILITY IN REPORTING CLINICAL GENETICS RESULTS TO FAMILIES}

Thompson $E^{1,2}$, Liebelt J', McGregor L', Perez-Jurado $L^{1,2}$, Le Blanc $S^{\prime}$, Borrie $S^{\prime}$, Bae $\mathrm{H}^{\prime}$, Barnett $\mathrm{C}^{1,2}$

${ }^{I}$ SA Clinical Genetics Service, Women's and Children's Hospital, North Adelaide, Australia

${ }^{2}$ University of Adelaide School of Medicine, Adelaide, Australia

Managing results of investigations is a challenge for medical practitioners particularly in clinical genetics where several family members may be tested. A serious problem is being unaware that a result has (or has not) returned. Many doctors manage results by booking the patient for a follow up appointment. We have a long waiting list for first appointments, so allocating clinic appointments for results 
is impractical. We have instituted the telephone results appointment system. Each patient is allocated a telephone results appointment date and time (morning or afternoon), usually about 3 months after the initial clinic appointment. Each clinical geneticist has designated times for a telephone results clinic. The clinical geneticist is given a list of patients to contact and the genetics files for each patient. The clinical geneticist is not to be interrupted during the session. Patients are contacted by phone, or an SMS may be sent asking them to call about results. A letter is written to the patient about the interaction. If a result is not yet back, it is a good reminder to us to check when it will be ready and rebook a telephone results date. This system has improved our efficiency in giving results to our patients. It also serves as an audit that letters and investigations have been done. It has given us a sense of security that results are not being overlooked. The number of calls from patients seeking results is significantly reduced because they know we will be in touch.

\section{CYTOGENETICS}

\section{DIFFERENT XP DUPLICATIONS IN TWO SIBLINGS RESULTING FROM RECOMBINATION OF A MATERNAL COMPLEX XP REARRANGEMENT}

Attwood $A^{\prime}$, Storer $R^{\prime}$, Buchhiem $R^{\prime}$, Smith $S^{\prime}$, Derwas $C^{\prime}$, Harvey $S^{\prime}$, Nicholl J' ${ }^{1}$ SA Pathology WCH, Adelaide, Australia

Two siblings were referred for SNP array studies with the clinical indication of short stature. Both siblings showed Xp duplications but of differing sizes. The female sibling carried a $1.8 \mathrm{Mb}$ duplication at chromosome $\mathrm{Xp} 22.33$ involving the SHOX regulatory region (CNE5, downstream); the male sibling a $0.8 \mathrm{mb}$ duplication which was contained within the sisters duplication but did not contain downstream SHOX regulatory regions. FISH studies on cultured cells indicated both the duplications were on the $\mathrm{X}$ chromosomes. Maternal SNP array analysis showed a complex rearrangement involving both a $1.8 \mathrm{Mb}$ duplication and $0.8 \mathrm{Mb}$ triplication concordant with the proband's Xp breakpoints. Maternal study also showed a unique $1.4 \mathrm{Mb}$ duplication containing the STS gene. MLPA studies confirmed the size, duplication and triplication of these Xp rearrangements. Intensive FISH studies performed on cultured maternal chromosomes and both siblings helped to clarify the maternal rearrangement and of the duplications in the probands. Duplications involving both upstream and downstream SHOX regulatory regions have been associated with SHOX related phenotypes; however, it is unclear in these patients given the lack of duplicated regulatory regions in the male proband that this is causative of short stature. The inheritance of different recombinants in the probands from the mother also adds to the complexity of these results.

\section{SNP MICROARRAY IN LOW-HYPODIPLOID ACUTE} B-LYMPHOBLASTIC LEUKEMIA - A CASE STUDY

\section{Battersby $\mathrm{S}^{\prime}$}

${ }^{I}$ Sydney Genome Diagnostics. Children's Hospital at Weastmead, Westmead, Australia

Background: Genome or chromosome 'ploidy' in B-cell acute lymphoblastic leukemia (B-ALL) has important prognostic implications. In B-ALL, high -hyperdiploidy has a favourable prognosis whereas hypodiploidy unfavourable. Hypodiploid ALL can go undetected as chromosome endo-reduplication or 'doubling' of the hypodiploid clone can result in a near-diploid or hyperdiploid clone. Aim: We present a 49-year-old female who was referred for 'New leukemia ALL'. Method: G-band karyotyping was performed on $24 \mathrm{hr}$ and $48 \mathrm{hr}$ synchronized bone marrow cultures, which were initiated and harvested according to standardized laboratory procedures. Chromosome microarray analysis was performed using the Illumina $300 \mathrm{~K}$ CytoSNP array at mean resolution $0.2 \mathrm{Mb}$ with $\mathrm{LOH}$ resolution $3-5 \mathrm{Mb}$. RT-PCR for the recurrent translocations $\mathrm{t}(1 ; 19)$, $\mathrm{t}(12 ; 21), \mathrm{t}(4 ; 11)$ and $\mathrm{t}(9 ; 22)$ was done elsewhere. Results: Initial karyotype analysis showed an abnormal hyperdiploid clone with 64-65 chromosomes with gains of 1, 2, 5, 6, 10, 11, 18, 19, 20, 21 and 22. SNP array analysis showed reduced $\log \mathrm{R}$ ratios and 'mosaic' loss of heterozygosity (LOH) for chromosomes 3, 4, 7, 8, 9, 13, 14, $15,16,17$ and X. Subsequent scoring of additional metaphase cells found a hypodiploid clone with 34 chromosomes with loss of X, 3, 4, $7,8,9,13,14,15,16$, and 17. The recurrent ALL translocations were not detected by RT-PCR. Discussion: The initial karyotype, and the absent ALL translocations suggested a standard risk classification for the patient. However, this case highlights the benefits of combined karyotype and SNP array to identify masked hypodiploidy in ALL, which reclassified this patient to high risk.

\section{PALLISTER-KILLIAN SYNDROME WITH MOSAIC HEXASOMY 12P ASSOCIATED WITH PIGMENTARY SKIN CHANGE}

Harvey $S^{\prime}$, Waters $W^{\prime}$, Hutchinson $R^{\prime}$, Eyre $H^{\prime}, Y u S^{\prime}$, Barnett $C^{2}$

${ }^{1}$ SA Pathology, Cytogenetics Laboratory, WCH, North Adelaide, Australia

${ }^{2}$ Paediatric and Reproductive Genetics Unit, WCH, North Adelaide, Australia

Pallister-Killian syndrome is a rare multisystem disorder characterized by isochromosome $12 p$ and tissue-limited mosaicism for tetrasomy $12 \mathrm{p}$. We report a 7 year old boy with significant developmental delay, right optic nerve hypoplasia and patchy pigmentary skin changes over several parts of his body. PallisterKillian syndrome was considered clinically as a likely diagnosis. Multiple cytogenetic investigations from birth showed no abnormality, including microarray studies on peripheral blood lymphocytes and FISH studies on buccal mucosal cells using a chromosome 12 centromeric probe. Skin biopsies were sampled from nonpigmented and pigmented skin. Chromosome analysis of cultured fibroblasts from non-pigmented skin showed hexasomy $12 p$ in $7 \%$ of metaphases examined. Chromosome analysis of cultured fibroblasts from pigmented skin showed hexasomy $12 \mathrm{p}$ in approximately $1 \%$ of metaphases examined. Microarray and FISH studies from these skin types confirmed these findings. This confirmed the clinical suspicion of Pallister Killian syndrome. Mosaic hexasomy $12 p$ has been reported three times in the literature. This presentation will compare our case with others in the literature.

\section{A RARE WHOLE-ARM TRANSLOCATION AND A QUESTION OF CENTROMERES}

Pollock $\mathrm{C}^{\mathrm{I}}$, Vipond $\mathrm{T}^{\mathrm{I}}$, Dunn $\mathrm{P}^{\mathrm{I}}$, Brookwell $\mathrm{R}^{\mathrm{I}}$

${ }^{1}$ Sullivan Nicolaides Pathology, Bowen Hills, Australia

Reciprocal chromosomal translocations are the most common structural rearrangement in humans and can often be familial. Apparent whole-arm translocations, however, are rare and result from the centromeric fusion of two chromosomes, followed by centric fission. We herein describe the case of a female who presented for conventional cytogenetics with reported recurrent miscarriages and was found morphologically to apparently have a whole-arm translocation between chromosomes 6 and 14. FISH centromeric studies were performed to determine the orientation of the centromeres. However, unusually, one of the derivative chromosome 14 was dicentric, while chromosome 6 possessed only part of the original centromere. This is therefore a very rare type of rearrangement, involving breakage of one centromere only, which raises the question of the processes involved in maintaining kinetochore function to sustain cell viability. 


\section{PHENOTYPIC VARIATION IN TWO RARE CASES OF MOSAIC TETRASOMY 9P}

Skeklios $A^{\prime}$, Bratkovic $D^{2}$, Brown $S^{\prime}$, Suwalski $S^{\prime}$, Smith $S^{\prime}$, Higgins $S^{\prime}$, Attwood $\mathrm{A}^{\prime}$, Nicholl J', Hutchinson $\mathrm{R}^{\prime}$

${ }^{I}$ Cytogenetics, SA Pathology, Adelaide, Australia

2 Paediatric Metabolic Clinic, SA Clinical Genetics Service, Adelaide, Australia

Tetrasomy $9 \mathrm{p}$ is a rare chromosomal abnormality involving an extra isochromosome. Both cases presented here have a karyotype which describes mosaicism for an additional isodicentric chromosome 9 (idic(9)(q12)). The first case is a 12 year old female with right hemi atrophy and mild scoliosis. Microarray studies detected a mosaic $40.6 \mathrm{Mb}$ terminal duplication at 9p24.3 - p13.1. Karyotype studies described an additional idic(9)(q12) in 18\% of lymphocytes examined. The second case is a 45 year old male with hypotestosteronemia and inconspicuous penis. A karyotype was performed to rule out sex chromosome abnormalities such as Klinefelter syndrome. Karyotype studies confirmed an additional idic(9)(q12) in $80 \%$ of lymphocytes examined. Array studies could not be performed. Most reported cases of tetrasomy $9 \mathrm{p}$ are mosaic. A review of sixty-six reported cases show the most common clinical features include: intellectual disability/development delay (73\%), abnormal ears (69\%), skeletal anomalies (55\%), hypertelorism (46\%), micro-retrognathia (45\%), genito-urinary or renal anomalies (43\%), bulbous nose (40\%), growth retardation (39\%) and cardiac defects $(37 \%)$. This syndrome can have a highly variable phenotype as five of the reported cases presented an entirely normal phenotype. The degree and distribution of mosaicism among different tissue types may contribute to these phenotypic differences. This is highlighted by our patients who presented with disconcordent phenotypes.

\section{17 YEARS OF CHROMOSOME BREAKAGE TESTING FOR FANCONI ANEMIA AT THE CHILDREN'S HOSPITAL AT WESTMEAD}

Sutedja $\mathrm{P}^{\prime}$, Wu $Z^{\prime}$, Sharma $\mathrm{P}^{\prime}$, Wright $\mathrm{D}^{\prime}$

${ }^{I}$ The Children's Hospital at Westmead, Westmead, Australia

Fanconi Anemia (FA) is an autosomal recessive chromosome breakage syndrome, characterized by DNA-crosslinking agent hypersensitivity and predisposition to bone marrow failure. Chromosome breakage testing (CBT) is recommended test for a diagnosis. We reviewed CBT over a $17-y r$ period to investigate the frequency of FA diagnoses. We present 5 cases of interest. The retrospective study period was 2000-2017. CBT involved scoring chromatid breaks and radial formation in 'treated' [mitomycin C (MMC) and diepoxybutane (DEB)] and 'untreated' blood lymphocytes cultures using sex and age-matched controls. A positive result was defined $>10$-fold breakage and chromosome fragility index $(\mathrm{CFI})>55$. Massive Parallel Sequencing using a panel (15 genes) was performed elsewhere. A total 465 CBT were performed. For the periods $2000-10$ vs. 201117, the mean CBT per year was 15 vs. 45. In 2013, testing increased 3 -fold ( 15 vs. 43 ) then 4 -fold (15 vs. 56) in 2017. Overall, 20/465 (4.3\%) FA positive cases were identified involving 12 vs. 9 cases for the two periods. Five interesting cases included one mosaic with abnormal karyotype/microarray results, and one with biallelic FANCD1 sequence variants. We observed 3-4 fold increased CBT since 2013, which can be attributed to (1) confirmation/exclusion of FA in aplastic anemia or MDS/AML patients, (2) requirement for a functional assay following MPS, and 3) few laboratories now perform CBT. Increased testing has not resulted in increased FA diagnostic cases. Five cases demonstrate that (1) FA can be accompanied by chromosome abnormalities, (2) CBT remains a functional assay, and (3) mosaicism can confound diagnosis.

\section{A COMPLEX VARIANT APL TRANSLOCATION $(3 ; 17 ; 15)$ ELUCIDATED BY CONVENTIONAL CYTOGENETIC AND FLUORESCENT IN SITU HYBRIDIZATION}

Wong $\mathrm{T}^{\mathrm{I}}$

${ }^{I}$ The Children's Hospital at Westmead, Westmead, Australia

Introduction: Acute promyelocytic leukemia (APL) is specifically characterized by the recurrent rearrangement $\mathrm{t}(15 ; 17)(\mathrm{q} 24 ; \mathrm{q} 21)$ and PML-RARA gene fusion. APL is found in $10 \%$ of adult acute myeloid leukemia and is associated with a favourable prognosis. Variant translocations occur less frequently, which can include complex rearrangements, however the clinical and morphological features are similar to typical APL. Herein, we describe a case of a 60 year old female who presented with pancytopenia. Method: The

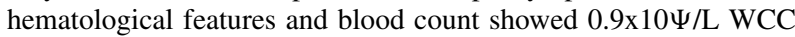
and $12 \times 10 \Psi / \mathrm{L}$ platelets with $33 \%$ circulating blasts. Marrow morphology showed blasts with open chromatin, nucleoli, scanty blue cytoplasm, folded nuclei, Auer rods and hypergranular cytoplasm. Immunohistochemistry markers were positive for CD34 and CD 117. 48-hour synchronized cultures were established for G-banded karyotyping. Rapid interphase and metaphase Fluorescent in situ hybridization (FISH) was performed using a dual fusion probe for PML-RARA. Results: Initial interphase FISH showed an atypical pattern with only a single fusion: nuc ish(PML,RARA)x3(PML con RARAx1)[65/100]. Follow-up karyotype and metaphase FISH studies showed $\mathrm{t}(3 ; 17 ; 15)(\mathrm{p} 21 ; \mathrm{q} 21 ; \mathrm{q} 24)$ and faint RARA and PML signals on chromosome $17 \mathrm{q}$ and chromosome $3 \mathrm{p}$, respectively. Conclusion: Initially, rapid interphase FISH studies showed an atypical single fusion signals, instead of dual fusion signals. Subsequent karyotype follow up showed an apparently balanced complex three-way translocation $\mathrm{t}(3 ; 17 ; 15)(\mathrm{p} 21 ; \mathrm{q} 21 ; \mathrm{q} 24)$, which is consistent with the metaphase FISH finding of a single PML-RARA fusion and apparent loss of the reciprocal RARA-PML fusion. Therefore, follow-up karyotype and metaphase FISH studies are both recommended to fully characterise atypical FISH signal patterns when found.

\section{GENETIC COUNSELING}

\section{HELPING FAMILIES FIND MEANING THROUGH THE (EVEN MORE) MEDICALIZED PROCESS OF AN UNDIAGNOSED DISEASES PROGRAM}

Broley $S^{\prime}$, Baynam $\mathrm{G}^{1,2,3}$

${ }^{1}$ Genetic Services of Western Australia, Perth, Australia

${ }^{2}$ Telethon Kids Institute, Perth, Australia

${ }^{3}$ Curtin University, Spatial Sciences, Perth, Australia

The Undiagnosed Diseases Program (UDP) in Perth is an interdisciplinary approach aiming to obtain a definitive answer for children and young adults with severe diseases who have escaped diagnosis despite exhaustive efforts over a prolonged period of time. With a renewed and intensive focus on clinical assessments, targeted investigations and new technologies through UDP participation, maintaining a focus on the psychosocial needs of families has been challenging. With the recent addition of a nurse coordinator to the team, the genetic counseling role within our team is evolving from core practical, coordinating tasks to a significant emphasis on family engagement, communication, resources and networking in the context of rare and undiagnosed diseases. With a diagnosis being identified for about half the families to date, many remain without an answer. Going forward, the fundamental focus of the UDP genetic counseling role is working towards an active, informative and ultimately rewarding process for families regardless of diagnostic outcome. 


\section{COMMON THREADS IN THE NSW GENETICS PATIENT} JOURNEY

\section{Culling $\mathrm{B}^{\prime}$, Packer $\mathrm{L}^{\prime}$, Curry $\mathrm{J}^{2}$}

$\bar{I}$ eHealth NSW, Gladesville, Australia

${ }^{2}$ DXC Technology Australia, Melbourne, Australia

Background: Currently NSW Health Clinical Genetics units use the Genetic Information system (GIS) a state-wide single database as well as the local facility Patient Administration System (PAS) / electronic Medical Record (eMR). An important tool for any clinical genetic service is the pedigree (family tree) with the ability to dismat. Visibility and access to information through the PAS and eMR ensures genetic information is not siloed and colleagues can access relevant information in a timely fashion. However using two separate systems means the patient record is dispersed and some work processes are duplicated. Methodology: Stakeholder engagement, primarily with service providers, was primarily through attendance at a one day workshop. Thirty-eight (38) participants, repical, genetic counseling and administrative staff) attended. The patient journey modelling tool known as Essomenic was used to model the current genetic patient journey this was validated, opportunities for improvement and alternative solutions identified. Outcome: We will present the stakeholder engagement, outcomes of this work and where this vision has progressed to. play information relating to diagnoses or genetic testing in this forresenting $14.5 \%$ of the clinical genetics workforce (including clin-

\section{WITHDRAWN}

\section{IMPACT OF VARIATIONS OF UNKNOWN SIGNIFICANCE RESULTS ON GENETIC COUNSELING PRACTICE FOR PRE-IMPLANTATION GENETIC DIAGNOSIS}

Kluvers $\mathrm{E}^{\prime}$, Mullen J'

${ }^{1}$ Monash IVF, Clayton, Australia

The impact of variants of unknown significance (VUS) on Preimplantation Genetic Diagnosis (PGD) genetic counseling in the IVF setting will be discussed in the context of policy development, legality and moral responsibilities. The advances in genetic testing techniques presented a unique challenge in counseling this subset of patients, including patients seeking advise on whether PGD is an appropriate option for the VUS. The incidence of VUS raises the question of how to best approach them in the practice of PGD. The National Health and Medical Research Council (NHMRC) 'Ethical Guidelines on the Use of Assisted Reproductive Technology in Clinical Practice and Research 2017' states 'PGT may only be used to select against conditions, disease or abnormalities that would severely limit the quality of the life of the person who would be born.' To ensure appropriate genetic counseling meets the individual need of each unique VUS, thorough investigations should be conducted to determine whether there is strong enough evidence to suggest that a specific VUS has the potential to cause harm and therefore meets the criteria for PGD. Monash IVF genetic counselors and consulting clinical genetics, together with the Monash IVF Medical Advisory Committee and Health Legal team, have addressed these issues with the development and implementation of a key policy outlining the clinics approach to VUS. This policy allows for effective genetic counseling of patients to help determine the best cause of action for each specific VUS and aid in answering the question; to screen or not to screen?

\section{POWER IN NUMBERS: AN ALLIANCE OF COLLABORATORS TO ADDRESS THE GENETIC COUNSELOR DEMAND IN ASIA}

Dow $\mathrm{R}^{\prime}$, Salmon $\mathrm{L}^{\prime}$, Graetz $\mathrm{M}^{2}$, Howat $\mathrm{P}^{3}$

${ }^{I}$ Genetics in the North East, Austin Health, Heidelberg, Australia

${ }^{2}$ Mercy Hospital for Women, Heidelberg, Australia

${ }^{3}$ The Northern Hospital, Epping, Australia

The Northern Hospital (TNH) in Epping is a major community hospital that provides antenatal and maternity health services for women in Melbourne's rapidly growing northern suburbs. In 2014, TNH began offering women and couples at increased risk of having a child with a genetic condition, genetic counseling services onsite. This was established as part of a collaboration with The Mercy Hospital for Women and Austin Health, Genetics in the North East. TNH genetic counselor (GC) coordinates the care and provides support for women who have a suspected abnormality in their baby. It was recognized that the clinical pathway for this patient group is often more complex and challenging than for those receiving care at a tertiary level maternity hospital. One of the major challenges is that unlike a tertiary centre, diagnostic testing and specialist opinion for TNH patients usually occurs outside the hospital. Adding to the complexity is the cultural and linguistic background of this patient group. Navigating this clinical pathway is critical to patient outcomes in this setting. Prior to TNH GC role, care for these women was fragmented with no consistent point of contact. We will describe the patient journey for women with a suspected fetal abnormality and highlight the critical nature of the TNH GC role in overcoming the challenges in delivering genetic counseling services in a nontertiary setting. Results regarding patient demographics, proportion of patients requiring appointments outside $\mathrm{TNH}$ and the outcomes for these patients will also be presented.
Laurino $M^{1,2}$, Cham $B^{3}$, Abad $P^{1}$, Lee $J^{4}$, Chu Y, Kejriwal $S^{5}$, Faradz $S^{6}$, Horiuch $\mathrm{Y}^{7}$, Bhatnagar $\mathrm{N}^{8}$, Chien $\mathrm{S}^{9}$

${ }^{1}$ University Of The Philippines Manila, Ermita, Philippines

${ }^{2}$ Seattle Cancer Care Alliance, Seattle, United States

${ }^{3}$ KK Women's and Children's Hospital, Singapore, Singapore

${ }^{4}$ Genetic Counselling Asia, Kuala Lumpur, Malaysia

5 Strand Life Sciences, Bengaluru, India

${ }^{6}$ Diponegoro University, Semarang, Indonesia

7 Tokyo Metropolitan Institute of Medical Science, Tokyo, Japan

${ }^{8}$ Board of Genetic Counseling India, Hyderabad, India

${ }^{9}$ Taichung General Hospital Genetic Consultation Center, Taichung, Taiwan

Fuelled by molecular technique advancements and increasing incorporation of diagnostic tools to identify genetic conditions, the global demand for genetic counselors to provide quality genetic counseling services continue to rise. In Asia, some countries (China, India, Indonesia, Japan, Malaysia, Philippines, South Korea, and Taiwan) are already offering graduate-level genetic counseling training programs, while others are actively drafting their justifications to develop their own. Acknowledging the varying curriculum and teaching modalities, efforts are ongoing to establish standardization of the 'genetic counselor' core skill requirements, scope of practice, and creating opportunities to maintain professional competency. As a step towards forming an alliance among Asian countries, the Professional Society of Genetic Counselors in Asia (PSGCA) leadership convened with representatives from the Board of Genetic Counseling - India, the Indonesian Society of Genetic Counselors, the Japanese Board of Genetic Counseling, and the Taiwan Association of Genetic Counseling on November 8, 2017, Bangkok, Thailand. This meeting was strategically planned at the completion of the 2nd 
Genetic Counseling Pre-Conference Workshop held prior to the 12th Asia Pacific Conference on Human Genetics wherein representatives from various countries in the Asia-Pacific shared their status of clinical genetic counseling services and training programs. In addition to the needs of increasing the number of practicing 'genetic counselors,' the professional issue to address the processes of genetic counselor certification, registration, or licensure in the Asia region has become critically urgent. Together, we strive to conduct the necessary work to develop the overarching professional standards that individual Asian countries can adopt.

\section{HOW FAMILIES USE SOCIAL MEDIA TO COMMUNICATE GENETIC INFORMATION}

\footnotetext{
Leighton $S^{1}$, Forrest $L^{2}$, Young $M^{3}$, Lynch $E^{4}$, Delatycki $M^{5}$

${ }^{1}$ Tasmanian Clinical Genetics Service, Hobart, Australia

${ }^{2}$ Parkville Familial Cancer Centre, Parkville, Australia

${ }^{3}$ Genome.One, Darlinghurst, Australia

${ }^{4}$ Melbourne Genomics Health Alliance, Parkville, Australia

${ }_{5}^{5}$ Victorian Clinical Genetics Services, Parkville, Australia
}

As genetic information may have health and reproductive implications for the proband and their family, assisting families to adequately communicate genetic risk information is vital. Previous research has explored the family communication process, often detailing barriers and facilitators to communication. This study aims to explore how families use social media to communicate genetic information to their relatives. This quantitative study employed a questionnaire to survey probands in families about their use of social media to communicate genetic information. Eligible participants included 323 probands from the reproductive carrier screening program at the Victorian Clinical Genetics Services and 250 probands from the Parkville Familial Cancer Centre who had hereditary cancer predisposition gene mutations. The 127 responses (response rate $22 \%$ ) showed that the majority of participants (79\%) did not use social media to communicate genetic information. Most participants preferred personal methods of communication, did not deem social media to be appropriate for such discussions and did not trust social media with their private and confidential genetic information. Those that did use social media ( $21 \%$ ) found it to be helpful as it was easy, accessible and allowed individuals to overcome communication barriers. For most, bypassing personal methods of communication in favour of social media may impact the efficacy of imparting genetic information. However, in certain circumstances, it may be practicable to assist people to use social media to communicate genetic information to their relatives. The findings provide further knowledge about the familial communication process and may assist genetic counselors to facilitate meaningful family dialogue.

\section{WITHDRAWN}

\section{IT'S A CURSE! ILLNESS PERCEPTION AND CAUSAL ATTRIBUTION IN AN ABORIGINAL FAMILY WITH MARFAN SYNDROME}

\author{
Mcinerney-leo $A^{1}$, West $J^{2}$, West $M^{2}$, Brown $M^{1}$, Duncan $E^{3}$, Meiser $B^{4}$ \\ ${ }^{1}$ Queensland University of Technology, Woolloongabba, Australia \\ 2 University of Queensland, Brisbane, Australia \\ ${ }^{3}$ Department of Endocrinology, RBWH, Brisbane, Australia \\ ${ }^{4}$ University of New South Wales, Sydney, Australia
}

Background: Illness representations and causal attributions are important determinants of how health threats are processed and how they affect health-related behaviors. Methods: 27 members of a large Aboriginal Australian family with Marfan syndrome were invited to participate in an ethically approved study exploring illness perception and causal attribution, including the perceived causes of phenotypic variability within the family. Semi-structured, qualitative interviews were recorded, transcribed verbatim and thematically an- alyzed. Results: 18 individuals participated. Marfan syndrome affected day-to-day living, and both affected and unaffected individuals reported many physical, psychological and logistical challenges. The severe vision impairment accompanying this syndrome was referred to as 'blindness', and this was perceived as the greatest challenge, permeating many aspects of life and contributing to feelings of stigma and exclusion, both in childhood and adulthood. The psychological and financial consequences of not being able to work were repeatedly mentioned. The majority knew that Marfan syndrome was genetic, and some perceived that the condition had been transmitted by a British ancestor as Aboriginal Australians were not believed to have the condition prior to that time. Further, some postulated that viruses or bacteria, such as syphilis, caused the mutation in the first place. The biggest factors perceived to be contributing to syndrome-related cardiac severity were alcohol and drug abuse. Conclusion: The considerable psychosocial and financial impact of Marfan syndrome was reported. Although, participants knew the genetics of Marfan syndrome, many hypothesized about the origin of the original de novo mutation. Gene-environment interaction was thought to affect severity.

\section{HOW FILIPINO PARENTS COPE WITH HAVING A CHILD WITH MAPLE SYRUP URINE DISEASE}

\author{
Tumulak $M^{\prime}$, Padilla $C^{1}$, Laurino $M^{1}$, Regalado $E^{2}$, Legaspi $A^{3}$, Ventura $E^{4}$ \\ ${ }^{1}$ University of the Philippines Manila, Philippines \\ ${ }^{2}$ University of Texas Health Science Center, USA \\ ${ }^{3}$ Bow Valley College, Canada \\ ${ }^{4}$ University of the Philippines Diliman, Philippines
}

Introduction: In the Philippines, maple syrup urine disease (MSUD) is the most common inborn error of metabolism (IEM). A family may experience stress, anxiety, sorrow, or feelings of helplessness when a child is diagnosed to have a genetic disorder that may lead to chronic care and disability. An illness affects not only the child, but the whole family as well. Methods: The phenomenological approach was utilized to capture the coping experience of parents with children diagnosed with MSUD. A total of 12 parents were interviewed using a semi-structured set of questions. Thematic analysis was done to analyze the interview transcripts. Results: Knowledge of MSUD, coping strategies used, and their advice for other parents were considered to be part of their coping mechanism when their child was diagnosed with MSUD. The parents used different coping strategies, such as information seeking, acceptance of their fate, and strengthening their belief in God, in order for them to be able to adapt to their situation. Conclusion: The findings of this study describe the different coping processes used by parents of children with MSUD. These results will help inform health care policies and genetic counseling guidelines in order to assist Filipino families in coping with the diagnosis and improve outcomes of their affected children.

\section{GENETIC EDUCATION \\ 57. EVALUATION OF AN EXTERNAL QUALITY ASSURANCE PILOT PROGRAM FOR THE QUALITY ASSESSMENT OF GDNA EXTRACTS}

Chai $S^{\prime}$, Pillay $N^{\prime}$, Tay $K^{\prime}$, Badrick $T^{\prime}$, Bennetts $B^{1,2}$, Horan $M^{\prime}$

${ }^{1}$ Molecular Genetics, Royal College of Pathologists of Australasia Quality Assurance Programs, St Leonards, Australia

${ }^{2}$ Sydney Genome Diagnostics, The Children's Hospital at Westmead, Sydney, Australia

The accuracy and quality of a genetic test directly depends on the quality of the initial DNA extract. All DNA diagnosing technologies require high quality genomic DNA (gDNA) for reliable data production. Thus, the efficient extraction of gDNA from human tissue is paramount for all downstream genetic applications. Current external quality assurance (EQA) programs for DNA extraction 
involves the EQA provider supplying a fresh single blood sample or tissue type to all participating laboratories. This is restrictive to participants who regularly extract DNA from other tissue material and from archived tissue samples. To address this, the RCPAQAP developed a pilot EQA program to evaluate DNA extractions from different tissue types. In this program, participants were required to submit DNA extracts from their own clinical specimens. The first trial was completed in 2016, which involved six laboratories submitting a total of 40 DNA extracts. In 2017, a pilot program was performed with 11 laboratories submitting a total of 55 DNA extracts for quality assessment. The RCPAQAP evaluated each DNA extract using three strategies; (1) DNA integrity, (2) multiplex PCR amplification of 5 gene loci, and (3) relative quantification of 11 gene loci using real-time PCR. Performance for each category was determined using a $z$ score. An overview of the quality assessment of gDNA extracts from the 2017 pilot program will be presented.

\section{INCREASING GP REACH, ENGAGEMENT AND EVALUATION OF FIRST TRIMESTER SCREENING ONLINE EDUCATION}

Cusack $M^{\prime}$, Saleh $M^{\prime}$, Middleton $E^{\prime}$, Dunlop $K^{\prime}$

${ }^{I}$ The Centre for Genetics Education NSW Health, Northern Sydney LHD, St Leonards, Australia

Background: The Centre for Genetics Education (CGE) and RACGP's GP online education activity on First Trimester Screening (FTS) for the 2014-2016 triennium was updated, based on GP focus groups, for the 2017-2019 RACGP triennium, adding Non-Invasive Prenatal Testing (NIPT) at the request of GP education providers and genetic health services. A communication strategy was developed to increase GP reach and uptake of the activity for 2017. Aim: To evaluate the impact of the communication strategy (reach and uptake), identify if learning needs were met and relevance of the FTS activity. Methods: Enrolments and activity completions were recorded by RACGP (2013-2017). On activity completion, GPs were offered the online evaluation form, rating learning needs and relevance. Results were compiled by RACGP as Evaluation Report (8/3/17-23/3/18). Results: In 2017, 626/1166 (54\%) enrolled GPs, completed the FTS activity (usual range 10-40\%); an increase of $148 \%$ enrolments and $157 \%$ completions compared to 2014 , where $246 / 469$ enrolled GPs completed the activity. There were 197 (20\%) more GP enrolments and 99 (19\%) more GP completions in 2017, than from 2014 to 2016. From March 2017 to March 2018, 737 evaluations were completed: 656 (89\%) GPs rated their learning needs entirely met; $672(91 \%)$ GPs rated the FTS activity entirely relevant and video case studies were highly recommended. Conclusion: The combination of targeted education development, communication strategy and RACGP partnership resulted in a significant increase in enrolments, completions and positive evaluations of the FTS activity by GPs during 2017. This is an effective approach to GP education.

\section{WITHDRAWN}

\section{THE MASTER OF GENOMICS AND HEALTH: A FLEXIBLE APPROACH TO PROFESSIONAL EDUCATION IN GENOMICS}

Hodgson J'

${ }^{I}$ University of Melbourne, Australia

The Master of Genomics and Health (nested suite), began at the University of Melbourne in February 2018. It has been specifically designed to prepare a workforce that is knowledgeable and skilled in the practical, ethical and socially responsible implementation of genomics. Students can choose to undertake the full two years of study (200 points) or exit with a Graduate Certificate (50 points) or a Graduate Diploma (100 points). A major strength of the program is that it can be personalized to upskill those already working in a wide range of science, health, community/policy settings as well as provide recent graduates with skills to gain employment across areas related to genomics. Students complement core subjects in genomics, ethics, communication skills and research with their choice of electives from a wide range of academic discipline areas. Secondyear students undertake internships in their area of interest and may elect to carry out a minor research project. Various study pathways will be outlined to demonstrate how the program can provide expertise in areas as diverse as health ethics, public policy, variant curation, bioinformatics, clinical trials and research. Possible future applications to professional certification and training processes will be highlighted.

\section{DEVELOPMENT AND EVALUATION OF AN INFORMATION BROCHURE IN PARENTS OF CHILDREN WITH CONGENITAL HEART DISEASE}

Hunt $A^{1}$, Hilton $D^{2}$, Barlow-Stewart $K^{\prime}$, Fleming J', Winlaw $D^{2}$, Blue $G^{2}$

${ }^{1}$ Discipline of Genetic Medicine, University of Sydney Medical School - Northern, St Leonards, Australia

${ }^{2}$ Heart Centre for Children, The Children's Hospital at Westmead, Westmead, Australia

Background: The causes of congenital heart disease (CHD) in children are complex and often unknown, leading parents to ask how and why this has happened. Genetic counseling that provides information and support has been shown to benefit these parents. Aims: (1) To develop and evaluate a brochure designed to improve parents' knowledge of CHD causation. (2) Where understanding is improved, assess the impact on parents' psychosocial functioning. Methods: Parents of children attending a preadmission clinic prior to elective cardiac surgery are being recruited at the Heart Centre for Children (HCfC). An information brochure was developed and assessed against several readability scales and piloted with seven parents. Assessments will occur at two time-points (pre- and postreceiving the brochure) using purpose-designed knowledge measures and validated psychological measures. Appropriate statistical analyses will be used to assess parents' knowledge and psychological functioning between the two time points. Results: The brochure was well received by pilot participants and considered relevant, informative and useful. They were satisfied with the content and the majority reported feeling reassured. Minor modifications were made to the brochure, which was reassessed to remain below the eighth grade reading level. Evaluation of the revised brochure has commenced at the HCfC with study participants. Discussion/Conclusion: The use of readability scales and pilot studies in the target population are important to ensure complex genetic information is correctly conveyed and received. Pilot data suggests that this brochure will enhance parents' basic knowledge of CHD causes and psychosocial functioning in the absence of formal genetic counseling.

\section{PATIENT UNDERSTANDING AND OUTCOMES FOLLOWING GENOMIC SEQUENCING IN CLINICAL CARE}

Kanga-Parabia $A^{1,2}$, Martyn $M^{1,2,3}$, Keogh $L^{2}$, Trainer $A^{4,5}$, Melbourne Genomics Health Alliance Community Advisory Group, Gaff $C^{1,2}$

${ }^{1}$ Melbourne Genomics Health Alliance, Parkville, Australia

${ }^{2}$ The University of Melbourne, Parkville, Australia

3 Murdoch Children's Research Institute, Parkville, Australia

${ }^{4}$ Peter McCallum Cancer Centre, Parkville, Australia

${ }_{5}^{5}$ Melbourne Health, Parkville, Australia

Comparative effectiveness studies of genomic sequencing frequently focus on clinical utility for management of patients with germline or somatic disorders. Here, we extend this to include patient impact through a pre- and post-test study. We administered surveys to patients or parents of patients receiving either germline or somatic genomic sequencing as part of clinical care. Categorical data 
is analyzed using descriptive statistics, and open-ended questions using content analysis. To date, response rates are $72 \%$ (659/912) at baseline and 53\% (160/301) at follow-up. 98\% (57/58) of participants with a diagnosis or actionable mutation correctly recalled their result. However, in those with an uninformative result, 7/27 (26\%) cancer patients and 4/56 (7\%) germline patients were either incorrect or unsure. Germline patients who obtained a diagnosis had a significant increase in perceived personal control (empowerment) ( 1.069 vs. $1.437, p=.0006)$; this was not evident for those who did not obtain a diagnosis ( 1.069 vs. $1.020, p=.4566)$. Interestingly, results with clinical utility may be not always be viewed positively by patients. 9/146 (6\%) participants exhibited decision regret, including 3 cancer patients with clinically actionable findings. Furthermore, 22 patients reported unanticipated consequences, with two who did not obtain a diagnosis surprised this was not the end of investigations. Differences between test outcomes valued by patients were evident across the 11 clinical subgroups and will be detailed. Though generally positive, our results shed light on those who may need greater attention and contribute to efforts to include patient outcomes in comparative effectiveness research.

\section{DEVELOPING A SURVEY TO GAUGE CURRENT AND FUTURE GENOMICS PRACTICE AND EDUCATION/TRAINING OF MEDICAL SPECIALISTS}

King $E^{1,2,3}$, McClaren $B^{1,2,3}$, Nisselle $A^{1,2,3}$, Gaff $C^{1,3,4,5}$

${ }^{1}$ Australian Genomics Health Alliance, Parkville, Australia

${ }^{2}$ Murdoch Children's Research Institute, Parkville, Australia

${ }^{3}$ University of Melbourne, Parkville, Australia

${ }^{4}$ Melbourne Genomics Health Alliance, Parkville, Australia

5 Walter and Eliza Hall Institute, Parkville, Australia

Background: The rapidly evolving field of genomics is increasing clinical applications relevant to all disciplines. Current genetic services cannot meet growing demand, requiring widespread adoption of genomics across all specialties, but it is unknown to what extent this emerging field will be incorporated into practice. The role of medical specialists in genomics is yet to be defined, nor do we know what educational support they require. Aim: To develop a survey to assess current and future genomic practice and education and training needs of Australian medical specialists. Methods: A modified Delphi method is being used to develop the survey. A question bank was curated from published scales in the literature, unpublished scales through collaborator networks, and crafting questions to address five domains that emerged from previous qualitative research in our group: participant, practice, proximity, preparedness and preferences. The Delphi expert group included medical and genetic specialists, genetics educators, and evaluators. Experts are providing feedback via online survey software on importance, relevance and clarity of questions in relation to the project aim and survey domains. Results/Outcomes: The question bank consisted of 45 items, sourced from nine instruments. Twenty-two experts were recruited from across Australia: six medical specialists, nine genetic specialists and seven genomic educators/evaluators will complete the Delphi process by August 2018. The draft survey will then be piloted with medical specialists. The final validated tool will be used nationally, and potentially internationally, to assess current and future genomics practice and education/training needs of medical specialists.

\section{LANDSCAPE ANALYSIS OF INFORMATION MATERIALS FOR PATIENTS RELATING TO GENOMICS AND GENOMIC MEDICINE}

Lewit-Mendes $M^{1,2}$, Pereira $K^{1,2}$, Dunlop $K^{1,3}$, Boughtwood $T^{1,2}$

${ }^{1}$ Australian Genomics, Parkville, Australia

${ }^{2}$ Murdoch Children's Research Institute, Parkville, Australia

${ }^{3}$ The Centre for Genetics Education NSW Health, St Leonards, Australia

In the Genomics in the Community Project, Australian Genomics and Patient Advocacy Groups (PAGs) collaborate to make reliable and current genomic information materials available to the community. Project workshops have established content themes, and identified key issues and concerns to inform material analysis and development. This landscape analysis aimed to evaluate the quality and content of publically available information materials about genomic medicine for consumers, to determine which materials could be used or adapted for the Australian context, and identify unmet needs. The analysis also aimed to engage PAGs in determining the most useful materials for the target audiences. Information materials were identified from desktop research and evaluated using a framework of criteria developed for this study, based on established tools for assessing patient educational materials and expert input. PAGs were then surveyed to evaluate the usefulness of a selection of the top 30\% of materials. The survey captured quantitative data and comments, which were analyzed using thematic analysis. There were 138 relevant information materials identified. When evaluated against the framework, the mean score was $60.0 \%$ where $75-100 \%$ : meets most criteria; $50-74 \%$ : meets some criteria; $<50 \%$ : did not meet criteria satisfactorily. PAGs reported issues with content complexity and volume, use of visual content and relevance to target populations. This analysis found that few materials satisfied all aspects of the defined criteria. We are now working to present approved materials on a website (genomicsinfo.org.au) and develop targeted materials for patients, and ultimately for the broader community, informed by landscape analysis results.

\section{COMPROMISED MOLECULAR DIAGNOSIS DUE TO UNDISCLOSED MEDICAL HISTORY}

\section{Martindale J', South $A^{2}$, Wardrop J', Gillman $C^{\prime}$, Drake $\mathrm{K}^{2}$}

${ }^{I}$ Wellington Regional Genetics Laboratory, Newtown, Wellington, New Zealand 2 Canterbury Health Laboratories, Christchurch, New Zealand

We report on the case of a 27 -year-old female patient referred by the local fertility service. Cystic fibrosis screening was requested for her and her partner, who had absence of vas deferens. No functionally significant CFTR variant was identified in the partner; however, in the female patient there was evidence of a low level (approximately $15 \%)$ of the p.(Phe508del) mutation. Owing to concerns regarding the possibility of sample contamination, investigation of other samples from which DNA was extracted on the same day was carried out but no potential source of the mutation was identified. QF-PCR analysis indicated the presence of a mixed genotype. The $\mathrm{CF}$ carrier test was reported as inconclusive and a repeat sample was requested. Analysis of a follow-up blood sample showed identical results to the initial sample. It subsequently came to light that the patient was treated for Philadelphia-positive ALL at four years of age and therefore, a stem cell transplant would be a plausible explanation for the mixed genotype identified. Additional work and liaison with the referring clinician is currently underway to establish whether the patient is a genuine $\mathrm{CF}$ carrier and the subsequent implications for her and her family. This case highlights the difficulties associated with genetics referrals accompanied by minimal clinical information and the need for consideration of the most appropriate sample types for genetic diagnosis when there is a relevant medical history. 


\section{EDUCATION ISN'T AN ANSWER FOR EVERYTHING:} PREPAREDNESS TO PRACTICE GENOMIC MEDICINE

\author{
Crellin $E^{1,2,3}$, McClaren $B^{1,2,3}$, Nisselle $A^{1,2,3}$, Gaff $C^{1,2,3,4,5}$, Metcalfe $S^{1,2,3}$ \\ ${ }^{1}$ Australian Genomics Health Alliance, Parkville, Australia \\ ${ }^{2}$ Murdoch Children's Research Institute, Parkville, Australia \\ ${ }^{3}$ University of Melbourne, Parkville, Australia \\ ${ }^{4}$ Melbourne Genomics Health Alliance, Parkville, Australia \\ ${ }^{5}$ Walter and Eliza Hall Institute of Medical Research, Parkville, Australia
}

Background: Genomics is forecast to transform the way certain diseases are diagnosed, treated and managed, but little is known about non-genetic medical specialists' preparedness to practice genomic medicine. Aim: To explore non-genetic medical specialists' views and expectations of genomic medicine and need for future education or training, using immunologists and rheumatologists as exemplars. Methods: Australian pediatric and adult immunologists and rheumatologists were purposively recruited from specialty societies, professional networks and via direct approach. Data were collected using semi-structured telephone interviews. Interviews were audiorecorded, transcribed verbatim, and inductively co-coded to compare and contrast participant perspectives. Results: To date, $16 \mathrm{im}-$ munologists and 10 rheumatologists have been interviewed. Most specialists anticipated genomics changing medical practice. Some resisted this change, questioning the clinical utility of genomics or its fit with existing clinical practices. Others embraced change, although not necessarily out of choice - they saw their colleagues aboard the genomics train and didn't want to risk being left behind. Many of these specialists were overwhelmed by the pace at which genomics was changing and felt ill-prepared to discuss it with patients. To become better prepared to practice genomic medicine, specialists needed a variety of supports, including greater access to genetic counselors, clinician-friendly databases, backing from scientists and research institutes, and basic education and training (Genomics ' 101 '). Conclusion: For genomics to be successfully integrated into non-genetic medical specialists' practices, a range of interventions are needed, one of which is education. No single solution exists!

\section{CO-DESIGNING THE UTS MASTER OF GENETIC COUNSELING PROGRAMME WILL INFORM THE FUTURE OF THE PROFESSION}

McEwen $\mathrm{A}^{\prime}$, McLean $\mathrm{J}^{2}$, Bull $\mathrm{C}^{3}$, Jacobs $\mathrm{C}^{1}$

${ }^{1}$ Graduate School of Health, University of Technology Sydney

${ }^{2}$ Institute for Interactive Media and Learning, University of Technology Sydney

${ }^{3}$ University of Technology Sydney

Genetic and genomic technology is rapidly being integrated into healthcare. Genetic counselors are perfectly placed to lead this change process. Taking the lead requires us to embrace change, be open to opportunities, envisage how our roles might change in the future and consider what new skills we might need to develop. The ability to adapt to change and uncertainty are essential skills for genetic counselors of the future. Being open to possibility at times of change is a core part of the co-design process we are using to develop a responsive, future-focused and emergent curriculum for the new Master of Genetic Counseling program at University of Technology Sydney (UTS). Using co-design we facilitated early engagement with the genetic counseling community to invite current and ongoing participation in the development of the program. This presentation will outline the key themes and ideas emerging from this process, including the initial consultation involving $25 \%$ of the membership of the Australian Society of Genetic Counsellors $(n=70)$ and from a co-design workshop in which we 'made' the present and future states of genetic counseling with our Curriculum Advisory Committee. The group included experts in genetic counseling, clinical genetics, genomics, ethics, psychology, Indigenous health, education and learning design. Key themes include the need for increasing diversity, both within the profession and in future roles, cultural safety and engagement, and the place of anxiety and uncertainty in genetic counseling. The findings from these activities contribute to broader discussions about the future directions of the genetic counseling profession.

\section{HOW DIFFERENT FORMS OF INFORMATION DELIVERY AFFECT THE GENETIC COUNSELING PROCESS}

\author{
Moore $K^{1}$, Kamien $B^{2}$, Shalhoub $C^{3}$, Freeman $L^{4}$, Berman $Y^{4}$, O'Donnell $S^{5}$, \\ Buckman $M^{6}$, Lynch $S^{7}$, Joyce $M^{1}$, Fleming $J^{\prime}$, Barlow-Stewart $K^{1}$, Hopper $B^{8}$ \\ ${ }^{I}$ Genetic Medicine, University Of Sydney, St Leonards, Australia \\ ${ }^{2}$ Genetic Services of WA, King Edward Memorial Hospital, Perth, Australia \\ ${ }_{3}^{3}$ Centre for Clinical Genetics, Sydney Children's Hospital, Randwick, Australia \\ ${ }^{4}$ Clinical Genetics Department, Royal North Shore Hospital, St Leonards, Australia \\ ${ }^{5}$ Clinical Genetics Department, Hunter New England Health, Newcastle, Australia \\ ${ }^{6}$ Tamworth Community Health, Hunter New England Health District, Australia \\ ${ }^{7}$ Clinical Genetics Department, Temple Children's University Hospital, Dublin, Ireland \\ ${ }^{8}$ Forster and Taree Community Health, Hunter New England Health District, Australia
}

Delivering information during genetic counseling sessions is an important part of practice, helping patients to understand more about a condition and its inheritance pattern and providing them with the tools to make decisions. There are diverse ways to deliver the information. Aims: (1) To evaluate whether using a video about inheritance is at least as effective as traditional genetic counseling in providing knowledge. (2) To evaluate any variation in efficacy across demographic groups. (3) To assess clinician attitudes regarding use of a video in genetic counseling sessions. Adults, attending genetic counseling regarding an Autosomal Recessive (AR) or Autosomal Dominant (AD) condition for the first time are being recruited from five genetics services. They will be randomized to receive either traditional genetic counseling or counseling with a video to explain inheritance (https://www.youtube.com/watch? $\mathrm{v}=\mathrm{Nv} 6 \mathrm{qU}$ UsKYodA\&t= $35 \mathrm{~s}$; https://www.youtube.com/watch?v=yANF0bxHpzU\&t=130s), and complete an online knowledge questionnaire pre- and postintervention on an iPad. Data will be analyzed using statistical tests (SPSS). Clinicians' views captured using semi-structured telephone interviews will be analyzed thematically. We will present the data collected using the knowledge questionnaires, whether they are useful in clinic, and clinician views on use of videos in clinic. We aim to determine whether the videos achieve equivalent or different knowledge outcomes as the traditional methods. Finding whether the videos are useful in the explanation of inheritance patterns is important as we move towards more accessible genetic testing frequently delivered by non-genetic professionals. This may assist the profession cope with the influx of questions from both patients and clinicians.

\section{THE NRP1 MIGRAINE RISK VARIANT ALSO SHOWS EVIDENCE OF ASSOCIATION WITH MENSTRUAL MIGRAINE}

Pollock $C^{\prime}$, Sutherland $H^{\prime}$, Maher $B^{\prime}$, Lea $R^{\prime}$, Haupt $L^{\prime}$, Firth $A^{2}$, MacGregor $E^{2}$, Griffiths L'

${ }^{1}$ Genomics Research Centre, Institute of Health and Biomedical Innovation, School of Biomedical Sciences, QUT, Kelvin Grove, Australia

${ }^{2}$ Clinithink Limited, Bridgend, UK, Bridgend, United Kingdom

Background: In 2016, a large meta-analysis brought the number of susceptibility loci for migraine to 38 . This study performed subtype analysis for migraine without aura and migraine with aura, but did not test the loci with respect to other subtypes, including menstrual migraine (MM). This study aimed to test the hypothesis that single nucleotide polymorphisms (SNPs) robustly associated with migraine are individually or collectively associated with menstrual migraine. Methods: Genotyping of migraine susceptibility SNPs was conducted using the Agena MassARRAY platform on DNA samples from 235 women diagnosed with menstrual migraine as per International Classification for Headache Disorders II criteria and 140 controls. Alternative genotyping methods including 
RFLP, pyrosequencing and Sanger sequencing were used for validation. Statistical analysis was performed using PLINK and SPSS. Results: Genotypes of 34 SNPs were obtained and investigated for their potential association with menstrual migraine. Of these SNPs, rs2506142 located near the neuropilin 1 gene (NRP1), was found to be significantly associated with menstrual migraine. Genomic risk scores were calculated for all 34 SNPs as well as a subset of 7 SNPs that were nearing individual significance. Overall, this analysis suggested these SNPs to be weakly predictive of MM, but of no prognostic or diagnostic value. Conclusions: Our results suggest that NRP1 may be important in the etiology of MM. It also suggests some genetic commonality between common migraine subtypes and MM. The identification of associated SNPs may be the starting point to a better understanding of how genetic factors may contribute to the MM subtype.

\section{PERCEPTION AND PREDICTORS OF GENETIC TESTING FOR CORNELIA DE LANGE SYNDROME (CDLS)}

\author{
Purvis $\mathrm{R}^{1,2,4}$, Noon $\mathrm{S}^{3}$, Conway $\mathrm{L}^{2}, \mathrm{Krantz} \mathrm{I}^{3}$ \\ ${ }^{1}$ The Peter MacCallum Cancer Centre, Melbourne, Australia \\ ${ }^{2}$ Arcadia University, Philadelphia, USA \\ ${ }^{3}$ The Children's Hospital of Philadelphia, Philadelphia, USA \\ ${ }^{4}$ The Royal Melbourne Hospital, Melbourne, Australia
}

Predictors of genetic testing have been investigated across many common conditions. Data on predictors of genetic testing as well as its value and impact within rare disease populations is comparatively scarce. Cornelia de Lange syndrome (CdLS) is a heterogeneous disorder routinely diagnosed through clinical examination. As with other incurable disorders, a molecular diagnosis does not alter management rendering the value of genetic testing subjective. We surveyed parents of children with CdLS to elucidate predictors and attitudes surrounding the usefulness and value of testing, and the impact testing decisions have. 87 participants were recruited via email through the CdLS Foundation to complete a quantitative online survey incorporating three validated scales assessing genetic testing knowledge and attitudes, and attitudes towards uncertainty. Data were compared using Chi-Squared and independent $t$ test analyses. Uptake of testing was associated with (1) increased satisfaction with the quality of information and emotional support provided $(p=.015)$; (2) elevated knowledge of testing $(p=.001)$; and (3) involvement of a genetic counselor $(p<.001) .67 .3 \%$ of testing parents indicated altered management as significant motivation $(N=35 / 52)$, suggesting that uncertainty around testing remains prevalent. Overall, $82.3 \%$ of parents stated that genetic testing was valuable. This value may be experienced through non-medical benefits, with testing parents reporting increased feelings of control, community inclusion and happiness as compared to non-testing parents. These findings reinforce the responsibilities genetic professionals have to adequately inform and support individuals through testing. Testing should be offered to rare populations even where management remains unchanged as parents stand to gain valuable non-medical benefits.

\section{ACRONYMS FOR GENETICS PRESENTATIONS}

\section{Regan $M^{\prime}$, Hunter $M^{\prime}$ \\ ${ }^{1}$ Monash Health, Clayton, Australia}

An initial task for a clinical genetics trainee is to learn the features of a number of common conditions such as Marfan syndrome, Noonan syndrome and Kabuki syndrome to name a few. A number of conditions are named after their clinical features such as CHARGE syndrome (Coloboma, Heart defect, choanal Atresia, growth Retardation, Genitourinary anomalies and Ears concave/deafness) and a number of other acronyms can be found on the internet. As the trainee matures into the second year, a more structured approach is required for developing a differential diagnosis list for a clini- cal presentation. Chapter two of the Oxford Desk Reference Clinical Genetics outlines the clinical approach to a number of presentations. Here we present a number of new acronyms for clinical presentations, loosely based on this chapter. It should be stated that while acronyms are very helpful for some individuals, this is not universal and careful consideration should be given before making acronyms mandatory. In developing the acronyms, we try to use a name that is in some way related to the presentation, rather than just 'ABCD...' or 'LMNOP'. We avoid the use of pejoratives; however, we try to add a humorous edge if possible. Some of these require a little stretch of the imagination. Acronyms for common presentations include: ANORECTOL, TVA (two in Swedish) BOIL (cysts) for polycystic kidney disease, BROAD PhalanGe, CAFÉ NOT BLACK, DIAFRAGM (hernia), CONgential HEART DISease, KAPUT COCHLEA, FLOPPIES, BRINK 5\% (cut-off for limb asymmetry), FAT NOGGIN, SMALL HEAD, TOF, and BIG SPROG (overgrowth).

\section{MORE ACRONYMS IN GENETICS}

Regan $M^{\prime}$, Hunter $M^{\prime}$

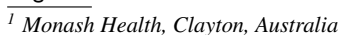

A new trainee in clinical genetics is confronted with a number of eponymous syndromes which need to be learned. Many of these are outlined in the FRACP clinical genetics syllabus and the $O x$ ford Desk Reference in Clinical Genetics. A trainee needs to be able to discuss these conditions intelligently and consider them as part of a differential diagnosis, despite never having seen the condition. As well as eponymous syndromes, there are a number of conditions named after the main signs and symptoms, such as VACTERL association (vertebral defects, anal atresia, cardiac defects, trachea-oesophageal-fistula, renal anomalies and limb abnormalities. Acronyms can be a good way to learn a large of amount of information for some people, but should not be made mandatory. We previously introduced new acronyms to help memorize the clinical features of common clinical genetics conditions with the following rules (1) avoid pejoratives, (2) common conditions, (3) use the eponymous name if possible, (4) avoid 'ABCE...' etc. and (5) include scoring systems if possible. Here, we further extend upon that work and introduce thirty new acronyms: ADAM Syndr, APERT, BARDET BIEDL, BATTEN, CANTU, LOWRY Syndr, COHEN, DIAMOND blackfan, ELLIS VAN C, FABRY S, FEINGOLD, FETAL alcohol, GAUCHER, GOLDENHAR, GORLIN, GRIEG, HIPPEL, MECP2, MOEBIUS, MUCOPOLYSACCharidosis, DySTROPIA, PEARSON, POLAND, POMPE, PROTUES, STEINER, STICKLER, TRECHER, TUBEROUS and WEAVER. With these new acronyms, pre-existing acronyms for named conditions (i.e., VACTERL) and acronyms available on the internet (i.e., MUSCLES for Duchenne Muscular Dystrophy) we hope to cover 100 common clinical genetics conditions.

\section{THE DEVELOPMENT AND DELIVERY OF A MASTERS GENOMICS STREAM AT THE UNIVERSITY OF MELBOURNE}

Reid $\mathrm{G}^{\prime}$, Charles $\mathrm{T}^{\prime}$

${ }^{I}$ Department of Paediatrics, The University of Melbourne, Parkville, Australia

Training in the area of genetic counseling has been offered by The University of Melbourne since 1996 when it was first offered as a graduate diploma. In 2008, in adherence with international recognition standards, the course was converted into a 2-year masters' program. In response to the rapidly advancing genomics era, a review of the Masters of Genetic Counseling (MGC) was undertaken in 2016/2017. During this time the curriculum was updated to reflect emerging technologies and practice, and in accordance with training guidelines outlined by the Human Genetics Society of 
Australasia. The delivery of course content was reconsidered in favour of incorporating a 'blended learning' approach. In addition to the revised MGC program, the Masters of Genomics and Health (MGH) was also launched through The University of Melbourne in 2018. The first of its kind in Australia, this qualification was developed in recognition of the need to prepare a workforce that is knowledgeable and skilled in the practical, ethical and socially responsible implementation of genomics. In 2018 there are 20 students enrolled in the MGC and 15 enrolled in the MGH. In this presentation, we will discuss the development and delivery of the genetics and genomics content of the two Masters' curriculums and how it is delivered as a nested suite of related subjects that utilise both online and face-to-face learning activities and assessments.

\section{JEWISH COMMUNITY GENETIC CARRIER SCREENING: ATTITUDES AND EXPERIENCES}

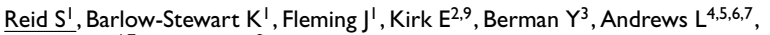
Cousens $\mathrm{N}^{4,7}$, Fleischer $\mathrm{R}^{8}$,

${ }^{I}$ Discipline of Genetic Medicine, University of Sydney Medical School - Northern, St Leonards, Australia

${ }^{2}$ Faculty of Medicine, Sydney Children's Hospital, Randwick, Australia

${ }^{3}$ Department of Clinical Genetics, Royal North Shore Hospital, St Leonards, Australia

${ }^{4}$ Hereditary Cancer Clinic, Prince of Wales Hospital, Randwick, Australia

${ }^{5}$ Nelune Comprehensive Cancer Centre, Randwick, Australia

${ }^{6}$ Wolper Jewish Hospital, Woollahra, Australia

${ }^{7}$ Prince of Wales Clinical School, University of New South Wales, Randwick, Australia

${ }^{8}$ Department of Medical Genomics, Royal Prince Alfred Hospital, Camperdown,

Australia

${ }^{9}$ School of Women's and Children's Health, University of New South Wales, Kensington, Australia
}

Introduction: People of Jewish descent are known to be carriers of mutations causing a number of recessive genetic diseases at higher rates than other populations. Screening programs have been implemented world-wide to provide carrier couples with reproductive options to avoid having an affected child. In Sydney, screening programs have been running in high schools since 1995, with alternative avenues of screening available through private hospitals and genetics services. Aims: To understand the ways in which genetic carrier screening is utilized and viewed in the Sydney Jewish community. This includes methods of screening that are popularly used, if and how genetic carrier results influence family planning, and if genetic screening is perceived as valuable by the community. It is expected that community genetic carrier screening continues to be positively received. Methods: Eligible participants (Jewish ancestry, aged 18-45) are being recruited for a voluntary, anonymous online survey (REDCap) through approving Sydney Jewish organizations. Survey items assess understanding of recessive inheritance patterns, and utilization of/ attitudes towards screening and carrier status. Statistical analysis will be conducted using SPSS. Preliminary Results: 33 surveys have been completed. 28 participants have undergone screening; $96 \%$ of those were satisfied with their overall experience, and $86 \%$ have or will consider their genetic status in family planning. Further findings will be compared with those from a 2003 study. Conclusion: Results of this study will have implications for the continued provision of community genetic screening programs, and may impact how health care providers offer genetic screening to Jewish individuals.

\section{A GENERAL PRACTICE (GP) ENGAGEMENT STRATEGY TO OPTIMIZE CARE FOR PEOPLE WITH NEUROFIBROMATOSIS}

\footnotetext{
Saleh $M^{1,2}$, Cusack $M^{1}$, Culnane $E^{3}$, Dabscheck $G^{3}$, Dunlop $K^{\prime}$

${ }^{I}$ The Centre for Genetics Education, St Leonards, Australia

${ }^{2}$ The Children's Tumour Foundation, Five Dock, Australia

${ }^{3}$ The Royal Children's Hospital, Parkville, Australia
}

Neurofibromatosis (NF) refers to a group of multisystemic genetic conditions including NF type 1, NF type 2 and Schwannomatosis.
The most common form of NF, NF type 1 (NF1) affects up to one in every 2500 people in Australia (Evans 2010, Ferner 2006). Despite this relatively high incidence, there is a lack of awareness and knowledge of NF among health care professionals and in the broader community (Crawford 2015, Barke 2016). In Australia, general practitioners (GPs) have the potential to improve early diagnosis and subsequent management of people with NF. The aim of this project is to develop a targeted communication strategy to raise awareness of NF and increase engagement and capacity building with the GP community. The outcome will focus on improved diagnostic confidence and an increase in GP knowledge of ongoing management and monitoring for NF. Since GPs are characteristically hard to reach and engage in education with varied success of educational approaches (Dadich 2016), a diverse and multifaceted approach will be employed to maximise its impact. Our strategy is adapted from a previously successful one used to raise awareness and uptake of a first trimester screening online activity for GPs. The GP strategy involves (1) key partnerships, (2) key messages/resources developed with target group (GPs) and (3) systematic dissemination to the key information/educational sites. This paper will report on the development of key messages and resources for GPs and its success in having them disseminated and engaging with the GP community throughout Australia.

\section{GENERAL PRACTITIONER ATTITUDES AND EXPECTATIONS FOR PROVIDING PERSONAL GENOMIC RISK INFORMATION TO THE PUBLIC}

Smit $A^{1,2,3}$, Newson $A^{2}$, Keogh $L^{4}$, Best $M^{2,5}$, Dunlop $K^{6}$, Voon Sen Tan $K^{7}$, Vuong $\mathrm{K}^{1}$, Kirk J ${ }^{8}$, Butow $\mathrm{P}^{9}$, Trevena $L^{10}$, Cust $A^{1,3}$

${ }^{1}$ Cancer Epidemiology and Prevention Research, The University of Sydney, Sydney, Australia

${ }^{2}$ Sydney Health Ethics, The University of Sydney, Sydney, Australia

${ }^{3}$ Melanoma Institute Australia

The University of Sydney, Sydney, Australia

${ }^{4}$ Melbourne School of Population and Global Health, The University of Melbourne, Melbourne, Australia

${ }_{5}^{5}$ Psycho-Oncology Co-operative Research Group (PoCoG), The University of Sydney, Sydney, Australia

${ }^{6}$ The Centre for Genetics Education, NSW Health, Sydney, Australia

${ }^{7}$ The University of Sydney, Sydney, Australia

${ }^{8}$ Westmead Clinical School, and Westmead Institute for Medical Research, Sydney Medical School, The University of Sydney, Sydney, Australia

${ }^{9}$ Centre for Medical Psychology and Evidence-based Decision-making, School of Psychology, The University of Sydney, Sydney, Australia

${ }^{10}$ Sydney School of Public Health, The University of Sydney, Sydney, Australia

Background: General practitioner (GP) attitudes towards anticipated future application of genomic knowledge for patient care are underexplored. Understanding these factors will be important for the development of strategies to prepare GPs for the expansion of genomics in primary care. Aim: To explore GP views of providing genomic risk information, their attitudes towards the potential provision of this information to the public and resources they feel that they require. Methods: We conducted semi-structured interviews inperson or via telephone with Australian GPs. The interviews were audio-recorded, professionally transcribed and analyzed thematically. Results: Twenty-two GPs participated in an interview; about half $(55 \%)$ were female. We identified several factors that may influence how and when GPs communicate genomic risk information. GPs understand genomics-based risk as one of many factors that influence individual disease risk and, therefore, believe that genomic risk information could be explained to patients within the context of overall risk assessment. Although GPs perceive themselves to be future gatekeepers of genomic testing, they recommended a shareddecision making approach. They believe that patients should: be able to interpret their genomic risk information, have the capacity to undertake relevant preventive behaviors, and not experience adverse psychological or emotional responses. GPs require clinical practice guidelines that specify recommendations for risk assessment and patient management, and evidence-based point-of-care resources and 
tools that incorporate genomic and traditional risk factors. Discussion/Conclusion: Our findings may assist with developing strategies and tools aimed at preparing the GP workforce, addressing information needs and encouraging appropriate implementation of genomics in primary care.

\section{VIEWS OF GENETIC SERVICE CLIENTS ABOUT THE GENETIC INFORMATION SYSTEM}

Speechly $C^{\prime}$, Berman $Y^{2}$, Barlow-Stewart $K^{1}$, Stenhouse $R^{2}$, Fleming $J^{\prime}$, Petrie $D^{3}$, Culling $\mathrm{B}^{4}$

${ }^{1}$ Discipline of Genetic Medicine, Sydney Medical School - Northern, University of Sydney, St Leonards, Australia

${ }_{2}^{2}$ Department of Clinical Genetics, Royal North Shore Hospital, St Leonards, Australia ${ }^{3}$ Genetic Alliance Australia, Darlinghurst, Australia

${ }^{4}$ Clinical Applications Support, Service Delivery, NSW Health, Gladesville, Australia

Background: The Genetic Information System (GIS) is a database of client information used across NSW and ACT genetic services. A recent study of genetic counselors' views about the GIS raised concerns about confidentiality and a perceived lack of client consent. However, views of genetic service clients about the GIS have not previously been identified. Aim: Using a qualitative focus group (FG) approach, we will explore the views of NSW and/or ACT genetic service clients about the type and use of information held in the GIS. Methods: FG participants will be adults, or carers of children, who previously attended a NSW or ACT genetic service and who respond to an invitation. They will be assigned to a FG and provided with a GIS resource prior to the session. The resource contains fictional scenarios and was developed to inform the exploration of views at the FG. The FG will be audio-recorded, transcribed, coded by three researchers, and analyzed using thematic inductive analysis. Data about participant demographics and past genetic service attendance will be collected and analyzed using descriptive statistics. Results: (1) The resource was piloted with 5 individuals and was well-received requiring minimal changes to content. (2) We will present the views of 6-30 participants attending 1-3 focus groups about how genetics services access and use information in the GIS for risk assessment of individuals and their genetic relatives. Discussion: Understanding client views may inform improvements to the GIS, and provision of educational resources to address any concerns identified.

78. OPPORTUNITIES TO DEVELOP STUDENTS' GENETIC LITERACY IN AUSTRALIAN SECONDARY SCHOOLS

Terrill B $^{1,2,3}$, Keast $\mathrm{S}^{4}$

${ }^{1}$ Kinghorn Centre for Clinical Genomics, Garvan Institute of Medical Research,

Sydney, Australia

${ }^{2}$ University of New South Wales, Sydney, Australia

${ }^{3}$ Genome.One, Sydney, Australia

${ }^{4}$ Monash University, Melbourne, Australia

Background: The changing needs of citizens and consumers in the genomic era have prompted new thought about teaching and learning genetics in formal education. As gene technologies enter mainstream healthcare, there is demand for accessible education to enable students to become 'genetically-literate' as informed health consumers and to participate effectively in societal discussions about genetic topics. Methods: We analyzed opportunities to develop students' genetic literacy at three levels in the Australian school system: the national curriculum, the state syllabus, and the classroom. In accordance with modified grounded theory, a qualitative document analysis, including frameworks, curriculum documents, and consultation correspondence, was combined with qualitative content analysis of three semi-structured interviews with Year 10 science teachers. Syllabus-aligned outlines of activities designed to support genetic literacy, including authentic data sets, contexts and argument-based exploration of socioscientific issues, generated to prompt teacher discussion. Results: Australian national standards now provide numerous opportunities to address genetic applications from Years 9-12. However, individual states and territories are responsible for schools education and teacher professional development, and ultimately, teachers determine how topics are addressed. Assessment requirements, accessible resources, teacher confidence and the lives, interests and abilities of their students have significant influence on the strategies teachers employed. Discussion/Conclusion: This study suggests an opportunity to develop students' genetic literacy in secondary schools in all states and territories, but that syllabus-aligned teacher resources and professional development across a range of topics will be key. These preliminary data now underpin development of Garvan Institute teacher resources for launch in 2018/19.

\section{CHALLENGES AND SUCCESSES IN RESEARCH GENETIC COUNSELING IN AN AUSTRALIAN NATIONAL PROGRAM}

Tom $M^{1,2}$, Edwards $S^{1,3}$, Boggs $K^{1,4}$, Wilkins $E^{1,5}$, de Silva $M^{1,5}$

${ }^{1}$ Australian Genomics, Australia

${ }^{2}$ Genetic Health Queensland, Brisbane, Australia

${ }^{3}$ Harry Perkins Institute of Medical Research, University of Western Australia Perth, Australia

${ }^{4}$ Sydney Children's Hospital Service, Sydney, Australia

${ }^{5}$ Victorian Clinical Genetics Services, Murdoch Children's Research Institute, Melbourne, Australia

Genetic counselors (GCs) adapts to the evolving needs of genetic testing technology and health services. These needs can be difficult to achieve in practise due to funding and resource restrictions, complex health systems and limited understanding of the role of GCs. In Australia roles for GCs in research have been limited. The Australian Genomics Health Alliance program, funded by the National Health and Medical Research Council to provide evidence for the equitable, effective and sustainable delivery of genomic medicine in healthcare, recently employed a number of GCs to provide clinical support and coordination nationally. These positions are funded by Australian Genomics but the GCs are employed by, embedded in, and managed by local health services. This study explored the practical, lived experience of clinical GCs who work in a research setting through this multi-site, multi-speciality program involving both genetics and non-genetics services. All eleven current Australian Genomics GCs were approached to participate. We utilized group discussions, surveys and semi-structured interviews within a phenomenological research paradigm to investigate their experiences and professional issues. Based across multiple states, GCs are employed full-time or part-time, and some have had research components integrated into their clinical roles. A range of themes relating to the overall program and individual health services were demonstrated from the above methods. The experiences described by GCs who participated in this study provide learnings that can support the integration of GCs into research design and implementation, and inform future service delivery and workforce planning.

\section{CONSUMER PERCEPTIONS AND EXPERIENCES REGARDING THE USE OF GENETIC INFORMATION IN INSURANCE}

Vargas Murillo B', Otlowski M², Doble $\mathrm{A}^{3}$, Barlow-Stewart $\mathrm{K}^{\mathrm{I}}$

${ }^{I}$ Discipline Genetic Medicine, University of Sydney Medical School - Northern

St Leonards, Australia

${ }^{2}$ Faculty of Law, University of Tasmania, Hobart, Australia

${ }^{3}$ FSC Genetic Testing Working Group, Sydney, Australia

Background: Internationally and in Australia there are concerns about the actual and potential occurrence of genetic discrimination (GD) in underwritten (risk-rated) insurance. Predictive genetic information is increasingly being generated by genomic technologies that are cheaper and offered both where there is a family history and in the context of wellness screening. Aim: To investigate consumer 
perceptions and experiences regarding the use of their genetic information in underwritten policy applications. Methods: The study will use a voluntary online survey containing questions about the participant's non-identifying demographic information (age, sex, postcode, relevant genetic condition), attitudes about genetic testing and insurance, and negative experiences they may have had when applying for different insurance products. Questions will address life and travel insurance products as well as private health insurance. The latter is included as there is anecdotal evidence of delays in initiation of cover based on their genetic test results. Asymptomatic consumers at the time of their experience (January 2010-December 2016) over 18 years with a personal or family history of an adult-onset genetic condition are being recruited from research institutes, relevant support groups and registries in Australia. Descriptive statistics will be used to analyze responses. Results/Conclusion: The results will provide an insight into the insurance experience and concerns about GD in that context among Australian consumers. Additionally, the study will inform understanding of barriers or coercion to have clinical or research genetic testing, examples of alleged negative treatment for consumers and barriers that may exist in regard to addressing the latter.

81. 'THERE'RE GOING TO BE QUITE DIVERSE ROLES': AUSTRALIAN GENETIC COUNSELORS' PERSPECTIVES IN THE GENOMICS ERA

Vuong $\mathrm{H}^{1,2,3}$, McClaren $\mathrm{B}^{1,2,3}$, Nisselle $\mathrm{A}^{1,2,3}$, Hickerton $\mathrm{C}^{1,2}$, Gaff $\mathrm{C}^{1,3,4,5}$

${ }^{I}$ Australian Genomics Health Alliance, Parkville, Australia

${ }^{2}$ Murdoch Children's Research Institute, Parkville, Australia

${ }^{3}$ The University of Melbourne, Parkville, Australia

${ }^{4}$ Melbourne Genomics Health Alliance, Parkville, Australia

${ }^{5}$ Walter \& Eliza Hall Institute of Medical Research, Parkville, Australia

Background/Aim: The utility of genomics to transform mainstream healthcare is being recognized by the Australian government. Genetic counselors (GCs) contribute crucial expertise to this change. Currently, few Australian studies have explored perceptions of GCs about the changing genomic landscape. Our project aims to: (1) explore the changing roles of GCs in relation to genomics in different service delivery models; and 2) assess the education and training needs of GCs. Methods: Semi-structured interviews are complementing survey data collected in 2017 from GCs who trained/currently work in Australasia. We are purposively sampling GCs Australia-wide who indicated they work clinically, both with and without genomics in their current practice, and who were willing to be interviewed $(N=35)$. Interviews are being audio-recorded, transcribed and analyzed thematically, using constant comparative method. Interviews will continue until data saturation is reached. Results: Interim data $(N=2$, with a further 3 scheduled) indicate that while GCs are cognisant of the significance of genomics to patients they also note patients may not see a difference between genetics and genomics. They note overlap between research and clinical practice ('It's an interesting time for the profession ... there're going to be quite diverse roles'), and perceive a lack of recognition of the GC skillset and contribution ('I find myself having to advocate ... for genetic counseling as a profession'). Discussion: These early themes suggest continuing education about unique genetic counseling implications in genomics is essential to patient care. Workforce support is also dependent on providing non-clinical training and increased inter-disciplinary collaboration and recognition.

\section{ONLINE BRCA SCREENING IN THE AUSTRALIAN} JEWISH COMMUNITY: A QUALITATIVE STUDY

\author{
Yuen J,2, O'Shea R', Cousens $\mathrm{N}^{3}$, Andrews $\mathrm{L}^{3,4}$ \\ ${ }^{I}$ Discipline Genetic Medicine, Sydney Medical School - Northern, St Leonards, \\ Australia \\ ${ }^{2}$ Cancer Genetics Service, National Cancer Centre Singapore, Singapore, Singapore \\ ${ }^{3}$ Hereditary Cancer Clinic, Prince of Wales Hospital, Sydney, Australia \\ ${ }^{4}$ Prince of Wales Clinical School, The University of New South Wales, Sydney, Australia
}

Background: Screening programs for founder BRCA mutations in the Jewish community have been advocated internationally. This is due to proven cost-effectiveness from its efficacy in decreasing morbidity and mortality of carriers by cancer screening strategies and risk-reducing surgery. An online website that delivers pretest information and obtains consent for BRCA testing using selfadministered cheek swabs is being trialed. Methods: Participants were invited via email to undergo two semi-structured, telephone interviews, at the pre- and post-result stage of online BRCA testing. Interviews were designed to explore their experience with the online BRCA testing process of obtaining pre-test genetic information, giving consent and receiving results. Interviews were transcribed verbatim and inductive content analysis conducted. Results: Of 43 participants, 12 consented to the interviews. Emerging themes identified are: Theme 1: Embracing shift to online testing: preference and satisfaction with online testing, reduced time, increased ease, accessibility and convenience, well-informed online decision, considerations for the future. Theme 2: Personalizing the experience: self-paced learning, stepwise information acquisition, learning process best person-tailored. Theme 3: Responsibilities to undergo testing: personal responsibility to test, results informative for health and family, contributing to community data. Conclusions: Our exploratory findings show that all participants are highly satisfied with online BRCA testing, for it increases the accessibility and convenience of testing, while allowing for personalization of the genetic testing process. This indicates that online BRCA screening programs could be a welcomed alternative to in-person genetic counseling in the Jewish population and presents a feasible model for future low-risk population testing.

\section{GENETIC EPIDEMIOLOGY}

\section{EVALUATING HYPERTROPHIC CARDIOMYOPATHY DISEASE-GENE ASSOCIATIONS USING THE CLINICAL GENOME RESOURCE (CLINGEN) GENE CURATION FRAMEWORK}

Ingles $\mathrm{J}^{\prime}$, Goldstein $\mathrm{J}^{2}$, Caleshu $\mathrm{C}^{3}$, Corty $\mathrm{E}^{2}$, Crowley $\mathrm{S}^{2}$, Dougherty $\mathrm{K}^{4}$ McGlaughon $J^{2}$, Milko $L^{2}$, Morales $A^{5}$, Seifort $B^{2}$, Semsarian $C^{1}$, Strande $N^{2}$, Thaxton $C^{2}$, Thompson $K^{6}$, van Tintelen $P^{7}$, Wallace $K^{2}$, Walsh $R^{8}$, Wells $Q^{9}$, Whiffin $\mathrm{N}^{8}$, Witkowski $\mathrm{L}^{10}$, Ware $J^{8}$, Hershberger $\mathrm{R}^{5}$, Funke $\mathrm{B}^{10}$

${ }^{1}$ Centenary Institute, Australia

2 Department of Genetics, UNC, Chapel Hill, USA

3 Stanford University, USA

${ }^{4}$ Eastern Virginia Medical School, USA

${ }^{5}$ Ohio State University, USA

${ }^{6}$ Oxford Medical Genetics Laboratory, Oxford, UK

${ }^{7}$ Academic Medical Center, Amsterdam, Netherlands

${ }^{8}$ National Heart and Lung Institute, Imperial College London, London, UK

${ }_{9}^{9}$ Vanderbilt University Medical Center, USA

${ }^{10}$ Department of Pathology, Massachusetts General Hospital and Harvard Medical School, Boston, USA

Background: Hypertrophic cardiomyopathy (HCM), defined as unexplained left ventricular (LV) hypertrophy is typically caused by variants in sarcomere genes. Current diagnostic gene panels often include $>50$ genes, with varying supportive evidence. Here we apply a systematic approach to assessing the validity of disease-gene associations. Methods: The ClinGen gene-disease clinical validity framework was used. The ClinGen HCM Gene Curation group comprises $>20$ international experts and biocurators. Genes were 
curated by biocurators, overseen by a cardiac expert, and discussed on regular calls. The gene list was based on (1) presence on $>30 \%$ HCM panels from the NCBI Genetic Testing Registry, (2) inclusion in the Illumina TruSight HCM panel, and (3) expert opinion. Genes were curated primarily for HCM, or syndromes including LV hypertrophy where appropriate. Results: Twenty-four gene panels were identified and included 162 unique genes, with only $23(14 \%)$ genes seen on $>50 \%$ of panels, and $58(36 \%)$ genes on a single panel only. We curated 58 genes, including 35 for an HCM phenotype, of which 8 were 'definitive' (MYBPC3, MYH7, TNNT2, TNNI3, TPM1, ACTC1, MYL2, MYL3), 1 'strong' (ALPK3), 3 'moderate' (CSRP3, TNNC1, JPH2), 16 'limited', and 7 'no evidence'. The largest group (66\%) had 'limited'/'no evidence'. Of 21 syndromic genes curated, 11 had 'definitive' evidence for isolated LV hypertrophy. Conclusion: While 8 genes have definitive evidence, HCM gene panels are largely comprised of unique genes with limited or no evidence of disease association. Systematic curation of HCM genes will guide appropriate reporting of variants.

\section{CHRONIC KIDNEY DISEASE PATIENTS WITH INHERITABLE KIDNEY DISEASE HAVE HIGHER ASSOCIATED HEALTHCARE COSTS}

\footnotetext{
Mallett $\mathrm{A}^{1,2,3,4}$, Sowa $\mathrm{P}^{4,5}$, Wardrop $\mathrm{L}^{2}$, Zhang $\mathrm{J}^{3,4}$, Cameron $\mathrm{A}^{3,4}$, Hoy $\mathrm{W}^{3,4}$

${ }^{1}$ Department of Renal Medicine, Royal Brisbane and Women's Hospital, Herston, Australia

${ }^{2}$ KidGen Collaborative, Australian Genomics Health Alliance, Parkville, Australia ${ }^{3}$ Faculty of Medicine, The University of Queensland, Herston, Australia

${ }^{4}$ Faculty of Medicine, The University of Queensland, Herston, Australia
${ }_{5}$ CHM CKD.CRE and CKD.QLD, The University of Queensland, Herston, Australia ${ }_{5}^{5}$ Centre for the Business and Economics of Health, The University of Queensland, Woolloongabba, Australia
}

Background: Those with autosomal dominant polycystic kidney disease (ADPKD) and non-ADPKD inherited kidney disease (IKD) are an identifiable minority of chronic kidney disease (CKD) patients. Their HSU in Australia are not defined. Aim: To identify characteristics and health service utilization among patients with CKD due to ADPKD, IKD and all-CKD causes in Queensland. Methods: Patients with ADPKD and IKD were identified from the CKD.QLD Registry $(n=7,541)$ and a matched Queensland Health CKD cohort $(n=22,129)$ based upon ICD-10 coding. Characteristics and HSU related to hospital presentations were analyzed for 5 years retrospective from June 2016. Results: 309 (1.04\%) ADPKD and $277(0.93 \%)$ IKD patients were identified from the combined cohort $(n=29,670)$. The median age at first admission of ADPKD, IKD and All-CKD patients were 53.7, 47.9 and 65.7 years respectively with mean eGFR at CKD.QLD consent being 34.1, 36.5 and $40.8 \mathrm{ml} / \mathrm{min} / 1.73 \mathrm{~m}^{2}$. ADPKD and IKD compared to All-CKD had lower frequency of diabetes ( $17 \%$ vs. $21 \%$ vs. $48 \%$ ) and cardiovascular disease (CVD; $34 \%$ vs. $32 \%$ vs. $46 \%$ ). ADPKD and IKD were associated with higher incidence than All-CKD of dialysis within that 5 year period ( $34 \%$ vs. $24 \%$ vs. $9 \%$ ). Median hospital admissions were higher in those with ADPKD or IKD compared to AllCKD (7vs8vs4) with similar average length of stay (3.2 vs. 4.4 vs. 3.6 days). Median 5-year healthcare costs were higher in those with ADPKD and IKD compared to All-CKD (\$42,088 vs. \$45,929 vs. \$16,403). Conclusions: Those with ADPKD and IKD are younger, with earlier stage CKD and lower prevalence of diabetes and CVD compared to other CKD patients. Despite this, they are more likely to require dialysis within 5 years and have increased hospital costs.

\section{THE PREVALENCE AND IMPACT OF INHERITED RENAL} DISEASE AT THE ROYAL MELBOURNE HOSPITAL

\author{
Talbot $A^{\prime}$, Trainer $A^{2}$, Winship $\left.\right|^{2,3}$, Nicholls $K^{1,3}$ \\ ${ }^{1}$ Department of Nephrology, Royal Melbourne Hospital, Parkville/Melbourne, \\ Australia \\ ${ }^{2}$ Department of Clinical Genetics, Parkville/Melbourne, Australia \\ ${ }^{3}$ Department of Medicine, University of Melbourne, Parkville/Melbourne, Australia
}

Background: Chronic kidney disease (CKD) affects $10 \%$ of the adult Australian population. Lifestyle factors including Obesity, Type 2 diabetes, and hypertension are major causes. Monogenic renal disease represents $\sim 10 \%$ of adult CKD population and a greater percentage of those reaching end-stage disease. Patients with monogenic or inherited renal disease are poorly defined, heterogeneous and frequently under-recognised. Autosomal dominant polycystic kidney disease (AD-PCKD) and Alport syndrome represent a large fraction of the inherited renal diseases currently identified. The new genomic technologies have enabled better identification of inherited renal diseases. We aimed to determine the prevalence and impact of these diseases on an Australian Nephrology unit. The project involved establishing a renal genetics clinic to facilitate this. Methods: The Royal Melbourne Hospital (RMH) Nephrology patient database was interrogated. Patients with presumed inherited conditions were identified and invited to attend the Renal Genetics clinic for further evaluation. Results: Diagnostic data was available on 9500 living patients. 744 patients were identified with possible monogenic disease including AD-PCKD (285), Alport Syndrome (108), Fabry Disease (99), Primary FSGS (85), Autosomal Dominant Tubulo-interstitial Disease (32), Tubolopathies (22), Tuberous Sclerosis (21), Nephrogenic Diabetes Insipidus (12) and Cystinosis (9). A subgroup of these patients, excluding PCKD, were also considered for whole exon sequencing (WES) to potentially determine the gene(s) responsible. Conclusion: Approximately $8 \%$ patients attending the RMH Nephrology department have a presumed inherited renal disease. Establishment of a renal genetics clinic is facilitating resource planning, furthering genetic diagnosis and counseling, cascade testing, reproductive choices and better treatment options.

\section{GENOMICS}

\section{GENETIC COUNSELING IN THE ERA OF ULTRA-RAPID GENOMIC TESTING: REFLECTIONS FROM A CLINICAL CASE}

Akesson $L^{1,2,3}$, Prawer $Y^{1}$, Lunke $S^{2,4}$, Krzesinski $E^{1,5}$, Stark $Z^{2,3,6}$, Hunter $M^{1,5}$

${ }^{1}$ Monash Medical Centre, Melbourne, Australia

${ }^{2}$ Victorian Clinical Genetics Services, Murdoch Children's Research Institute, Melbourne, Australia

${ }^{3}$ Department of Paediatrics, University of Melbourne, Melbourne, Australia

${ }^{4}$ Department of Clinical Pathology, University of Melbourne, Melbourne, Australia

${ }_{5}^{5}$ Department of Paediatrics, Monash University, Melbourne, Australia

${ }^{6}$ Australian Genomics Health Alliance

Background: Genetic counseling in the era of genomics has undergone significant changes to deal with additional variants, more potential outcomes and implications of data storage and reanalysis. With the advent of ultra-rapid genomic testing via the Acute Care Genomics AGHA flagship, we reflect on how counseling must adapt with new challenges. Case Report: A term neonate born to consanguineous parents was transferred to a tertiary perinatal centre with respiratory insufficiency requiring positive pressure support and profound hypotonia and weakness. Initial investigations for common causes of hypotonia and weakness were normal. Results: In the clinical setting of minimal improvement, ultra-rapid genomic testing identified a rare homozygous pathogenic variant in RYR1. Discussion: In the neonatal intensive care setting, families often have limited time to adjust to critical illness before the concept of whole exome sequencing is introduced, which may reduce 
their ability to process complex information and make informed decisions about genetic investigations. Preliminary information giving and consent is time-pressured, with less opportunity to reflect on the implications of testing prior to making a final decision. A focus on potentially positive benefits of genomic testing, such as identifying a treatable disease or diagnosing a condition with a favourable prognosis, combined with rapid receipt of results, may limit families' ability to prepare for potentially devastating genetic diagnoses. Professionals involved in facilitating ultra-rapid genomic testing should be mindful of the potential to preferentially focus on these positive outcomes and adapt counseling to promote families' psychological adjustment to results and minimise the impact of diagnoses.

\section{MANAGING THE UNEXPECTED: A RETROSPECTIVE REVIEW OF OFF-PHENOTYPE GENETIC TEST RESULTS FROM CARDIAC GENE PANELS}

Allen $E^{\prime}$, Taylor J', Thompson $T^{\prime}$, Bogwitz $M^{\prime}$, Zentner $D^{1,2}$, Vohra J,2, Badman $\mathrm{S}^{\prime}$, Dow $\mathrm{E}^{\prime}$, Winship I', James $\mathrm{P}^{\prime}$

${ }^{1}$ Cardiac Genetic Clinic, Royal Melbourne Hospital, Parkville, Australia

${ }^{2}$ Department of Cardiology, Royal Melbourne Hospital, Parkville, Australia

Cardiac gene panels are now being routinely used in the clinical setting. With the implementation of this technology, patients are now undergoing genetic testing for a broad spectrum of genes associated with a variety of cardiac phenotypes. This increases the chance of an off-phenotype finding. As clinicians, we need to assess how to ethically handle these results. For patients, findings of this nature can be confusing and have the potential to increase anxiety. They also carry ramifications for other family members that need to be considered and addressed. For the purposes of this retrospective audit, an off-phenotype finding was defined as a mutation that is identified that does not clearly explain the phenotype, but would be considered clinically actionable. This obviously raises challenges for both patients and clinicians alike. Here we present a small number of cases seen through The Royal Melbourne Hospital Cardiac Genetics Clinic where genetic testing using a panel approach has detected an off-phenotype pathogenic (or likely pathogenic) result. We will discuss how these cases were handled, the challenges raised for both the patient and clinician, and the long-term recommendations given to these patients. Through this audit and in reviewing the literature relating to additional findings, we will consider the impact of offphenotype findings on patients and clinicians alike, and the genetic counseling provided both before and after testing. By highlighting the issues that have arisen with panel based genetic testing, we can be better informed as we transition into future technologies such as whole exome sequencing.

\section{GENOMIC TESTING IN AN OUTREACH SETTING: EXPERIENCE OF THE ACT GENETIC SERVICE}

Badman $S^{\prime}$, Bates $K^{\prime}$, Cook $M^{\prime, 2}$, Dopita $B^{\prime}$, Engel $A^{\prime}$, Freckmann $M^{\prime}$, Hogan $J^{\prime}$, Koina $E^{1,2}$, Warwick $L^{\prime}$

${ }^{I}$ ACT Health, Canberra, Australia

${ }^{2}$ Australian National University, Canberra, Australia

Genomic testing (whole exome or whole genome sequencing) is increasingly used in clinical genetics for the molecular diagnosis of suspected Mendelian disorders. Genomic testing is reliant on a multidisciplinary approach, with the combined expertise of genetic counselors, clinical geneticists, medical specialists, and laboratory scientists. In outreach services, where multidisciplinary team members are located in different cities, finding innovative methods of collaborating may be required. The ACT Genetic Service operates as an outreach service. There are 4.2 FTE genetic counselors based in Canberra, and clinical geneticist support from three clinicians based in Sydney. Recent funding has enabled development of the Canberra Clinical Genomic Service (CCGS): a collaboration between ACT Health and the John Curtin School of Medical Research (ANU).
CCGS has provided an opportunity for the ACT Genetic Service to offer an increased number of genomic tests, and prompted our service to examine how to best facilitate genomic testing in an outreach setting. We present an initial summary of clinical and research genomic tests ordered through the ACT Genetic Service in the past year. Data will include indication for testing, type of genomic test, laboratory, and results. We present processes for genomic testing in our service, strategies for managing challenges particular to this setting, and outline the importance of the genetic counselor role in an outreach service. We hope documentation of our experience will be valuable to other regional services providing genomic testing.

\section{INTERNATIONAL APPROACHES TO IMPLEMENTATION OF GENOMIC MEDICINE}

\author{
Belcher $\mathrm{A}^{\mathrm{l}, 2}$, Ward $\mathrm{R}^{1,2,3,4}$ \\ ${ }^{1}$ Australian Genomics, Australia \\ ${ }^{2}$ The University of Queensland, Brisbane, Australia \\ ${ }^{3}$ Faculty of Medicine, The University of New South Wales, Sydney, Australia \\ ${ }^{4}$ Global Genomic Medicine Collaborative (G2MC)
}

In recent years the need for multinational collaboration to facilitate genomics medicine implementation has been identified. The aim of this study was to identify national policies, frameworks, strategies or large-scale demonstration projects that are informing implementation of genomics. Several strategies were used to identify relevant initiatives including searches of the Web of Science-core collection, PubMed and Google Scholar along with searches of organizational plus federal and provincial government websites. Activities have been identified from around 30 countries with some countries having more than one activity. The most common activity type is demonstration projects followed by the development of policy frameworks and then population-specific variation studies. A review of the policy areas considered by the activities shows that the most common themes relate to improving scientific understanding of the genetic basis of disease or pharmacogenetics and the development of technical capabilities such as sequencing platforms. Following these are a group of themes including workforce skilling, Ethical, Legal and Social Implications (ELSI), information management, clinical and laboratory issues and communication and public education. It is hoped that making this information available through a catalogue will promote greater sharing of the learnings from these activities which will support faster and more consistent integration of genomics into health systems.

\section{GENETIC COUNSELING IN THE ERA OF GENOMICS:} WHAT'S ALL THE FUSS ABOUT?

Brett $\mathrm{G}^{1,2}$, Wilkins $\mathrm{E}^{1,2}$, Creed $\mathrm{E}^{1,3,4}$, West $\mathrm{K}^{1,3}$, Jarmolowicz $\mathrm{A}^{1,2}$, Valente $\mathrm{G}^{1,4}$, Prawer $Y^{\prime}, 5$, Lynch $E^{\prime}$, Macciocca $I^{2}$

${ }^{1}$ Melbourne Genomics Health Alliance, Melbourne, Australia

${ }^{2}$ Victorian Clinical Genetics Services, Murdoch Children's Research Institute, Melbourne, Australia

${ }^{3}$ Melbourne Health, Melbourne, Australia

${ }^{4}$ Genetics in the North East, Austin Health, Melbourne, Australia

${ }_{5}^{5}$ Monash Genetics, Monash Health, Melbourne, Australia

Background: As genomic sequencing becomes more widely available in clinical settings for diagnostic purposes, a number of genetic counseling issues are gaining precedence. The ability to manage these issues will be paramount as genetic and non-genetic healthcare professionals navigate the complexities of using genomic technologies to facilitate diagnosis and inform patient management.

Methods: Counseling issues arising when counseling for diagnostic genomic sequencing were identified by four genetic counselors with ten years of collective experience providing genetic counseling in this setting. These issues were discussed and refined at a meeting of genetic counselors working in clinical genomics settings in Melbourne, Australia. Illustrative cases were selected where pre- and post-test genetic counseling was provided in clinical 
settings to individuals who received singleton or trio whole exome sequencing with targeted analysis. Case summaries were compiled by the author providing counseling to the individual or family, using detailed consult notes recorded in a research database, which included reflections regarding psychosocial issues. Counseling issues raised and challenges for the counselor were extracted to ascertain the applicability of existing genetic counseling competencies to managing counseling issues arising. Outcomes: Counseling issues discussed include a reappraisal of how genetic counselors manage hope in the genomic era, informed consent for secondary use of genomic data, unexpected or unsolicited secondary findings, and trio sequencing. The authors seek to contribute to the evolving understanding of genetic counseling for diagnostic genomic sequencing through considering the applicability of existing genetic counseling competencies to managing emerging counseling issues, and discussing genetic counseling practice implications.

\section{WITHDRAWN}

\section{PUF60 AND VERHEIJ SYNDROME: A CASE REPORT AND LITERATURE REVIEW}

Fennell $A^{1,2}$, Regan $M^{1,2}$, Tan $N^{1,2}$, Prawer $Y^{1,2}$, Lunke $S^{3,4}$, Tan $T^{3,4}$, Hunter $M^{1,2}$

${ }^{1}$ Monash Genetics, Monash Health, Richmond, Australia

${ }^{2}$ Department of Paediatrics, Monash University, Clayton, Australia

3 Victorian Clinical Genetics Service, Murdoch Children's Research Institute, Melbourne, Australia

${ }^{4}$ The University of Melbourne, Melbourne, Australia

Children with intellectual disability and multiple congenital anomalies can be a difficult group to diagnose with vast clinical and genetic heterogeneity. Until the advent of whole exome sequencing (WES), a genetic basis usually went unknown. WES has been successful in expanding the list of ID-associated genes and is rapidly becoming the standard of care for this clinical group. We present a 5-year-old boy with marked global developmental delay, bilateral optic colobomas, pre-axial polydactyly, congenital heart disease and dental anomalies. Karyotype and chromosome microarray were normal. EEG was non-specifically abnormal. WES was performed using Nextera Rapid Capture Exome kit (Illumina) with $>100 x$ mean coverage. Data was processed using Cpipe and a phenotype-driven gene list for variant prioritization and assessed as per the ACMG variant classification guidelines. A novel heterozygous mutation in PUF60 (c.1154_1166del) was found resulting in a frameshift mutation. Subsequent segregation studies found this variant was a de novo variant in the proband and it was classified as pathogenic. Verheij syndrome is a neurodevelopmental syndrome due to interstitial deletions of 8q24.3, which includes the PUF60 gene. Recent reports implicate variants in PUF60 as causing a similar phenotype to Verheij syndrome. A literature review revealed 18 such cases now documented with markedly heterogenous phenotypes. The PUF60 syndrome phenotypic spectrum has been established to include: developmental delay/intellectual disability, unilateral ocular coloboma, microcephaly, short stature, craniofacial, cardiac and renal defects. We describe a case with the unique constellation of bilateral ocular coloboma, pre-axial polydactyly, proximal insertion of the right hallux and dental anomalies.

\section{DIGITAL KARYOMAPPING PROVIDES HIGHER SNP DENSITY FOR USE IN PREIMPLANTATION GENETIC DIAGNOSIS USING EMBRYONIC SEQUENCING}

Hardy $T^{1,2}$, Byrne $A^{4}$, Lawrence $D^{1,4}$, Arts $P^{4}$, King-Smith $S^{4}$, Babic $M^{1}$, Barnett $\overline{\mathrm{C}^{5}}$, Ledger $\mathrm{W}^{3}$, Scott $\mathrm{H}^{1,4}$

${ }^{1}$ SA Pathology, Adelaide, Australia

${ }^{2}$ Repromed, Dulwich, Australia

${ }^{3}$ University of New South Wales, Sydney, Australia

${ }^{4}$ Centre for Cancer Biology, University of South Australia, Adelaide, Australia

${ }^{5}$ SA Clinical Genetics Service, Women's and Children's Hospital, North Adelaide, Australia

Background: Genome-wide linkage methods for PGD such as arraybased karyomapping and haplarithmisis rely on informative single nucleotide polymorphisms (SNPs) which have been identified in population-based genotyping studies. Whole genome sequencing (WGS) allows the identification of SNPs which are unique to a family and may provide additional informative markers to facilitate linkage-based PGD by WGS of embryos. Research Question: Can SNPs identified using WGS provide high-resolution linkage analysis in a model of digital karyomapping? Methods: Model of linkage-based PGD using publicly-available genomes and manual analysis of inheritance vectors. CEPH Family 1463 genomes were accessed via the Platinum Genomes site and variants assigned to grandparental genotypes (NA12889, NA12890, NA12891 and NA12892) using a digital karyomapping approach. Results: A total of 2,290,211 informative variants could be assigned to grandparental genotypes. Informative variants were evenly distributed between each grandparent $(575,740,570,955,575,180$ and 568,336 variants from NA12899, NA12890, NA12891 and NA12892 respectively). Average SNP density was greater than that routinely achieved by karyomapping using SNP array (1 informative SNP per 1255 base pairs). Standard karyomapping of selected genes demonstrated an average 3225 informative variants per gene. Use of intragenic SNVs did not provide adequate targets for analysis of all grandparental haplotypes, necessitating a genome-wide linkage approach. Conclusion: Analysis of SNPs unique to an individual family using WGS can create a personalized list of informative variants which have a greater density than standard SNP arrays. Genome-wide linkage analysis of SNPs will increase the number of potential informative targets detectable using whole genome sequencing of embryos.

\section{EXPLORING MUTATIONS IMPLICATED IN SEVERE REACTIONS TO TRIVIAL HEAD TRAUMA}

Omar Ibrahim' ', Heidi Sutherland', Larisa Haupt' , Lyn Griffiths'

${ }^{I}$ Genomics Research Centre, Institute of Health and Biomedical Innovation, School of Biomedical Sciences, Queensland University of Technology

Background: Ion channel genes (e.g. CACNA1A) have mutations implicated in the development of severe neurological disturbances in response to trivial head trauma. However, a portion of the population presents with similar symptoms and have no mutations in the known genes. Aim: To explore pathogenic causal mutations of severe concussion or neurological disturbance following mild head trauma focusing on Ion Channel genes. Methods: This study utilized a population of 25 patients who presented with severe concussion symptoms following minor head injuries and who previously tested negative for mutations using the Genomics Research Centre FHM 5-gene diagnostic panel. Whole Exome Sequencing (WES) was performed on the Ion Proton (Life Technologies/ThermoFisher Scientific) using the Ion AmpliSeqTM Exome RDY Library Preparation and Ion PITM Hi-Q TM Chef Kit protocols. The Ion Reporter server was used for bioinformatics analysis. Results: Analysis was performed using filters that excluded variants deemed by Sorting Tolerant from Intolerant (SIFT) and Polymorphism Phenotyping (PolyPhen) scores to be benign and included only genes related to neural processes and which are highly expressed in the brain. 
Preliminary results suggest the implication of rare and novel mutations in Potassium Channel and Neurotransmission related genes. From the population, a father and a son had an unusual presentation, where after minor head trauma they developed multiple concussions, a 'migraine like' episode with slurred speech, diplopia, headache, and vomiting. Sanger sequencing validation is ongoing. Discussion: Potassium channels and Neurotransmission pathway genes may play a role in response to head trauma and could provide insights into the mechanisms of Traumatic Brain Injury (TBI).

\section{ESTABLISHMENT OF A MULTIDISCIPLINARY CARDIAC GENOMICS CLINIC: A ROADMAP FOR PRECISION MEDICINE?}

Johnson $\mathrm{R}^{1}$, Devery $\mathrm{S}^{2}$, Jabbour $\mathrm{A}^{1,3,4}$, MacDonald $\mathrm{P}^{1,3,4}, \mathrm{Wu} \mathrm{K}^{2,4,5,6}$, Fatkin $\mathrm{D}^{1,2,3,4}$

${ }^{I}$ Victor Chang Cardiac Research Institute, Sydney, Australia

${ }^{2}$ Clinical Genomics Unit, St Vincent's Hospital, Sydney, Australia

${ }^{3}$ Cardiology Department, St Vincent's Hospital, Sydney, Australia

${ }^{4}$ St Vincent's Clinical School, Faculty of Medicine, UNSW, Sydney, Australia

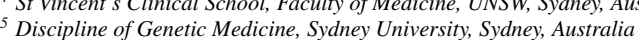

${ }^{6}$ Garvan Institute of Medical Research, Sydney, Australia

The St Vincent's Hospital Cardiac Genomics Clinic is a multidisciplinary clinic involving cardiologists, molecular cardiologists, clinical geneticists and genetic counselors at St Vincent's Hospital, with close ties to cardiovascular researchers at the Victor Chang Cardiac Research Institute (VCCRI). We provide a comprehensive service encompassing genetic testing and risk management, cardiac screening/management, pharmacogenomics, and research opportunities. The cardiac service is open to all individuals with a cardiac condition that is suspected to have a genetic basis who have been referred by their cardiologist, GP or other specialist. Genetic testing offered includes single gene tests, targeted gene panels, wholegenome sequencing, and pharmacogenomics as appropriate. The Cardiac Genomics Clinic is held once a month and since September 2017 we have seen 23 patients with a range of cardiac conditions, the majority having dilated cardiomyopathy or Long QT syndrome. Clinical genetic testing was conducted for 18 individuals (diagnostic testing in 10 probands and predictive testing in 8 relatives). Of the remaining 5 patients, 2 did not attend and 3 did not qualify for testing. Moving forward, multidisciplinary clinics will be a cornerstone of precision medicine, providing a one-stop shop for patients. Our clinic helps to addresses an unmet need for patients with heritable cardiovascular genetic disorders who historically have had poor access to genetic testing due to a lack of appropriate specialized services and funding. The close association with VCCRI provides a reciprocal clinical-research pathway for elucidating the pathogenicity of VUSs that are increasingly found through genomic testing, and for data-mining in unsolved cases.

\section{VARIANTGRID: AN OPEN SOURCE VARIANT DATABASE WITH GRAPHICAL ANALYSIS}

Lawrence $\mathrm{D}^{1,4}$, Feng J', King-Smith $\mathrm{S}^{3,5}$, Kassahn $\mathrm{K}^{3}$, Scott $\mathrm{H}^{1,2,4}$, Geoghegan J', Schreiber $\mathrm{A}^{1,4}$

${ }^{I}$ ACRF Cancer Genomics Facility, Centre for Cancer Biology, SA Pathology, Adelaide, Australia

${ }_{2}^{\text {Australia }}$ Centre for Cancer Biology, Department of Genetics and Molecular Pathology, an SA Pathology and University of South Australia Alliance, Adelaide, Australia

${ }^{3}$ Genetic and Molecular Pathology, SA Pathology, Women's and Children's Hospital,

North Adelaide, Australia

${ }^{4}$ School of Biological Sciences, University of Adelaide, Adelaide, Australia

${ }^{5}$ Australian Genomics Health Alliance, Australia

In this contribution, I will discuss VariantGrid, a variant database and web application developed for storing and analyzing Next Generation Sequencing data. VariantGrid is designed to scale to thousands of samples and billions of genotypes and is used in research analysing large cohorts of rare diseases and cancer, and for trios and singletons in SA Health diagnostics. It can also be used as a variant and classification sharing platform. VariantGrid performs vari- ant and gene annotation, manages multiple versions thereof as they are updated over time, facilitating comparisons and automatic reanalysis. The web front end allows managing data and tasks related to sequencing, patients, phenotypes, analysis and classification. Analyses can be locked down to gene lists and standardized filters for diagnostics, or users can create custom filters with a dragand-drop interface. A recent focus has been variant classification, and I shall discuss entering and storing evidence, what parts of the ACMG guidelines can be automated, as well as our methods of auditing and re-analysing classifications and sharing them between labs. I will also share our experience collecting and auditing years of historical classifications from 5 different diagnostic labs inside SA Health.

\section{PREPARING THE HEALTH SYSTEM FOR MAINSTREAMING OF GENOMICS: LESSONS LEARNED FROM EARLY ADOPTERS}

\author{
Long J', Paul J,5, Best $S^{1}$, Taylor $N^{3}$, Martyn $M^{2,4,5}$, Gaff $C^{2,4,5}$ \\ ${ }^{1}$ Australian Institute of Health Innovation, Macquarie University, Sydney, Australia \\ ${ }^{2}$ Melbourne Genomics Health Alliance, Melbourne, Australia \\ ${ }^{3}$ Cancer Council, Sydney, Australia \\ ${ }^{4}$ Murdoch Children's Research Institute, Melbourne, Australia \\ University of Melbourne, Melbourne, Australia
}

Background: Implementing genomics into mainstream healthcare will require an understanding of the psychosocial, environmental, and policy related factors affecting local clinical practice to design an effective implementation strategy. We interrogated data from interviews with 'early adopters' of genomic testing to examine underlying constructs driving behavior. Methods: Interviews were conducted with 32 participants involved in Melbourne Genomics' Demonstration Projects in 2015: clinical geneticists, genetic counselors, and specialist physicians. Participants spoke of their current experience of using genomics. Here we focus on their views around future mainstreaming of genomic testing: any behavior related to future envisioned practice when funded clinical testing is more widely available. We used the Theoretical Domains Framework (TDF), which is based on psychosocial theories of behavior change, to identify and group barriers and enablers to future mainstreaming of genomic testing. Results: Barriers were identified in 11 of the 14 Domains. Prominent issues included the need for genetic oversight of the process despite the unpopularity of the gatekeeper model among physicians (Domain: Behavioral Regulation); risks around unrealistic expectations, and patient harm from test result misinterpretation, and that without specialist genetic input, the test would not be cost effective (Beliefs about Consequences). Geneticists perceived physicians generally as overestimating their genetic knowledge (Beliefs about capabilities) and that the complexity of testing means that it requires time doing it to build up expertise (Knowledge/Skills). Discussion: Key issues will need to be addressed before genomic testing becomes mainstream. This analysis is being used to initiate evidence-based support for future applications of genomics.

\section{MOST CLINICAL DIAGNOSES OF EHLERS-DANLOS SYNDROME CANNOT BE CONFIRMED BY MOLECULAR TESTING}

Xue $Y^{1}$, Oh $M^{1}$, Wilcox $W^{2}$

${ }^{1}$ Fulgent Genetics, Temple City, United States

${ }^{2}$ Emory University, Atlanta, United States

Background: The Ehlers-Danlos syndromes (EDS) are a heterogeneous group of connective tissue disorders characterized by joint hypermobility, tissue fragility and skin hyperextensibility. The newly released 2017 international classification of EDS recognizes 13 subtypes based on clinical criteria (PMID: 28306229). The diagnosis of EDS, except hypermobile EDS, relies on molecular testing due to the genetic heterogeneity and phenotype overlap 
among EDS subtypes. Methods: Next Generation Sequencing for sequencing and deletion/duplication analysis was performed on 100 consecutive clinical EDS cases requested at Fulgent Genetics. Molecular testing results and clinical information, if provided, were reviewed. Results: To our surprise, the diagnostic rate was only $5 \%$. Interestingly, all the detected pathogenic variants were either in COL5A1 or COL5A2 with a triple helical glycine change in either genes. There was one case with two pathogenic variants in both COL5A1 and COL5A2. Variants of unknown significance were found in 14 cases. Discussion: The main reason for such a low molecular diagnostic rate for clinical EDS is probably because no genes have been identified for the most common hypermobile EDS subtype. In addition, the criteria for diagnosing hypermobile EDS may not be sufficiently specific so the over-diagnosis of this subtype in clinic is possible. Furthermore, most individuals would not meet the diagnostic criteria for classic EDS where mutations in COL5A1 and COLA2 account for about $50 \%$ of molecular diagnoses (ref). The new EDS classification may help solve these issues. The expanded gene panel including less common causes of EDS did not increase the molecular diagnostic rate.

\section{THE UTILITY OF NIPT IN PREGNANCIES COMPLICATED BY CO-TWIN DEMISE}

Shi G', Giouzeppos $\mathrm{O}^{\prime}$, Flowers $N^{\prime}$, Tsegay $A^{\prime}$, Harrington $T^{\prime}$, Manser $R^{\prime}$, Burns $I^{\prime}$, Baeffel $S^{\prime}$, Archibald $A^{1,2}$, Scarff $K^{1}$, Hunt $C^{\prime}$, Pertile $M^{1,2}$

${ }^{I}$ Victorian Clinical Genetics Services, Murdoch Children's Research Institute,

Parkville, Australia

${ }^{2}$ Department of Paediatrics, University of Melbourne, Parkville, Australia

Background: Non-invasive prenatal testing (NIPT) has been rapidly adopted as a highly accurate screening test for fetal aneuploidy in pregnancy. Co-twin demise in pregnancy is usually a contraindication for NIPT. Placental cytotrophoblast from the demised twin continues to release cell-free DNA (cfDNA) into the maternal circulation. This can lead to false-positive results and misreporting of fetal sex. Aim: We sought to use our experience in testing pregnancies with co-twin demise to inform our advice around management and testing of these pregnancies. Methods: We have screened pregnancies with ultrasound evidence/known co-twin demise using percept NIPT. Pregnancies with a 'false-positive' or 'misreported' sex have been retested at a later gestation to monitor the contribution of the demised twin to the cfDNA. Results: Of 120 pregnancies with evidence for co-twin demise, 27 had repeat NIPT at a later gestation. 18 cases showed a decrease in Y-chromosome counts in the repeat NIPT, suggesting a surviving female fetus. Our data show that cfDNA contribution from the demised twin decreases on average $1 \%$ per 4 weeks post demise. In addition, 15/120 pregnancies showed evidence for a fetal trisomy $(9,11,13,15,16,18,21,22)$. Conclusion: In our experience, cfDNA from a demised twin can persist for up to 8 weeks post-demise, but this is dependent on the gestational age at demise. NIPT is more likely to return a false-positive result when demise occurs from 8 weeks of gestational age. Where there has been a known co-twin demise, this clinical information can be used to inform when NIPT should occur.

\section{ADAPTATION OF ORGANIZATIONS THROUGH A} GENOMIC LENS

Ryan $\mathrm{J}^{1,2,3}$, Craig J $\mathrm{J}^{4}$, Hayes $\mathrm{P}^{1,2}$, Jones $\mathrm{S}^{1}$

${ }^{1}$ RMIT University, Melbourne, Australia

2 University of Melbourne, Melbourne, Australia

${ }^{3}$ Flinders University, Adelaide, Australia

${ }^{4}$ Deakin University, Melbourne, Australia

In a rapidly changing modern business environment, it is imperative that adaptive genomic mechanisms are maximized to capitalise on the organization's inherent adaptive capacity. However, the authors argue that in the modern business environment these mechanisms are being stretched such that they are now becoming maladaptive in their expression impacting negatively on health and well-being in the workplace. The authors suggest that for organizations to evolve and progress, there needs to be a fundamental paradigm shift in leadership thinking to focus on the health and well-being of both employees and leaders, in their relationship to each other and the natural environment. This requires understanding of genomic health and the factors relevant to the individual's genomic health and wellbeing (e.g., sleep, diet, exercise). This also requires understanding the nature of interplay between genomic health and organizational elements (e.g., leadership, structure, culture, strategy). Given the array of research initiatives aimed at understanding contributors to disease through the genomic lens, one can assume the connection between the workplace, the genome, and its associated impact on health and well-being (both physical and psychological) is beginning to unfold. This underlines the importance of genomic health as an issue of organizational and leadership responsibility. The authors will explore this challenge for the modern business world through the integrated lenses of biological and social sciences. They will investigate the role genomics can play in creating organizations and business environments that enable its members to adapt and thrive rather than just cope.

\section{SAPATHOLOGY'S CLINICAL GENOMICS JOURNEY: WHAT HAVE WE LEARNED, WHAT NOW, AND WHAT'S NEXT?}

Soubrier J', Gao $S^{\prime}$, Lawrence D², Fletcher J', Kassahn K'

${ }^{1}$ SA Pathology, Adelaide, Australia

${ }^{2}$ ACRF Cancer Genomics Facility, Centre for Cancer Biology, SA Pathology and University of South Australia, Adelaide, Australia

Introduction: Although genomic testing is now current practice across Australia there remain many challenges in providing a quality diagnostics service. Method: The first step to identify and tackle any potential issues is to obtain a detailed overview of both our testing requirements and efficiency and how they evolved over time. To this end, we have performed a comprehensive audit of high throughput sequencing (HTS)-driven diagnostic output over the past 4 years. Results: The number of tests performed through HTS has exploded in the years 2014-2015 but has since remained surprisingly stable. In the same time, the number of tests sent away to other laboratories has decreased slightly, but has also shifted in content with the inclusion of tests that were previously not offered at all. The lab is still performing a significant amount of Sanger tests, not only for family cascade screening but also for variant confirmation and to gap fill poorly covered regions with HTS. The workflow has changed from many off-the-shelf capture kits to a single custom design, allowing more cost-effective batching practices. Now we are seeing more specialization again, taking advantage of newly implemented techniques such as amplicon-based panels with molecular barcoding, or long-range PCR combined with HTS. Conclusion: Reflecting back on the evolution of genetic testing in the past few years and considering the ongoing technological progress in the field, the main ongoing challenges for clinical genomics in routine diagnostics remain providing timely, cost-efficient, helpful reports that answer the clinical question being asked. 
102. ARE WE READY FOR RAPID GENOMIC TESTING IN ACUTE PEDIATRICS? ATTITUDES OF AUSTRALIAN HEALTH PROFESSIONALS

Stark $Z^{1,2,3}$. Nisselle $A^{1,2,3}$. McClaren $B^{1,2,3}$ Lynch $F^{1,2,3}$, Best $\mathrm{S}^{4}$, Long $J^{4}$ Martyn

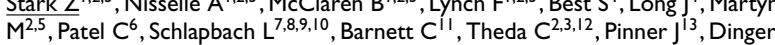
$M^{14,15,16}$, Lunke $S^{2,3}$, Gaff $C^{1,2,5,17}$

${ }^{I}$ Australian Genomics Health Alliance, Melbourne, Australia

${ }^{2}$ Murdoch Children's Research Institute, Melbourne, Australia

${ }^{3}$ University of Melbourne, Melbourne, Australia

${ }^{4}$ Australian Institute of Health Innovation, Macquarie University, Sydney, Australia

${ }^{5}$ Melbourne Genomics Health Alliance, Melbourne, Australia

${ }^{6}$ Genetic Health Queensland, Royal Brisbane and Women's Hospital, Brisbane, Australia

${ }^{7}$ Paediatric Critical Care Research Group, Mater Research Institute, The University of Queensland, Brisbane, Australia

${ }^{8}$ Paediatric Intensive Care Unit, Lady Cilento Children's Hospital, Brisbane, Australia

${ }^{9}$ Faculty of Medicine, The University of Queensland, Brisbane, Australia

${ }^{10}$ Department of Pediatrics, Bern University Hospital, Inselspital, University of Bern,

Bern, Switzerland

${ }^{11}$ Paediatric and Reproductive Genetics Unit, Women's and Children's Hospital,

Adelaide, Australia

${ }_{12}$ Neonatal Services, Royal Women's Hospital, Melbourne, Australia

${ }^{13}$ Sydney Children's Hospital, Sydney, Australia

${ }^{14}$ Garvan Institute of Medical Research, Sydney, Australia

${ }^{15}$ Genome.One, Sydney, Australia

${ }^{16}$ St Vincents Clinical School, UNSW Sydney, Sydney, Australia

${ }^{17}$ Walter and Eliza Hall Institute, Melbourne, Australia

Aim: To investigate the attitudes of healthcare professionals towards the application of genomic testing in neonatal and pediatric intensive care units (NICU/PICU). Methods: Before initiating a multicentre implementation study of rapid genomic testing across 18 Australian NICUs/PICUs, we surveyed 289 participating intensive care physicians and genetics professionals about perceived usefulness of results, preferences for service delivery models, and implementation readiness. We examined the confidence of intensivists with microarray and genomic testing, and preferences for professional development in genomics, and conducted semi-structured interviews. Results: The overall response rate was 58\%, $46 \%$ for intensivists (79/170) and 75\% (89/119) for genetics professionals. Intensivists reported only moderate confidence with using microarray tests and even lower confidence with genomic tests. Twentythree percent reported having engaged in genomics-related professional development activities. The majority of intensivists (76\%), clinical geneticists $(87 \%)$ and genetic counselors $(81 \%)$ favored a clinical genetics-led service delivery model of genomic testing in the NICU/PICU. Among clinicians who favored an intensivist-led model, result interpretation was highlighted as a key area likely to require additional support from clinical genetics. Perceived utility of genomic results was lower in the intensivist group compared to the genetics professionals group ( $20 \%$ vs. $40 \%, p=.024)$. While support for implementation was high, the main concerns expressed by genetics professionals related to implementation climate and organizational readiness. Conclusion: Successful large-scale implementation of genomic testing in NICU/PICU will require the development of interdisciplinary service delivery models that support adoption of genomics by the intensive care workforce while ensuring safety, sustainability and efficiency.

\section{DMD SPLICING VARIANTS INCREMENTALLY DIMINISHING LEVELS OF WILD-TYPE DYSTROPHIN DEFINE CLINICAL SPECTRUMS MYALGIA TO DMD}

Waddell $L^{1,2}$, Lek $M^{3}$, Bryen $S^{1,2}$, Bournazos $A^{\prime}$, Evesson $F^{1,2,4}$, Dawes $R^{1,2}$, Joshi $\mathrm{H}^{1}$, Jones $\mathrm{K}^{1,2,5}$, Tukianen $\mathrm{T}^{6,7}$, Weisburd $\mathrm{B}^{7,8}$, Valkanas $\mathrm{E}^{6,7,8}$, Cummings $\mathrm{B}^{6,7,8}$, Saddedin $S^{9}$, Marshall $J^{6,7,8}$, Davis $M^{10}$, Gooding $R^{10}$, Sandaradura $S^{1,2,5}$, Neas $K^{\prime \prime}$, Mowat $D^{12}$, Sampaio $H^{13}$, Oates $E^{14}$, Tchan $M^{15,16}$, O'Grady $G^{17}$, Arbuckle $S^{18}$, Graf $N^{18}$, Peters $\mathrm{G}^{19}$, Clarke $\mathrm{N}^{1,2}$, MacArthur $D^{6,7,8,20}$, Cooper $\mathrm{S}^{1,2,4}$

${ }^{1}$ Institute for Neuroscience and Muscle Research, Kids Research Institute, CHW, Westmead, Australia

${ }^{2}$ Discipline of Child and Adolescent Health, University of Sydney, Westmead, Australia

${ }^{3}$ Yale University, New Haven, USA

${ }^{4}$ Children's Medical Research Institute, Westmead, Australia

5 Clinical Genetics, Children's Hospital at Westmead, Westmead, Australia

${ }^{6}$ Analytic and Translational Genetics Unit, Massachusetts General Hospital, Boston, USA

${ }^{7}$ Medical and Population Genetics, Broad Institute of Harvard \& MIT, Boston, USA

${ }^{8}$ Center for Mendelian Genomics, Broad Institute of Harvard \& MIT, Boston, USA

${ }^{9}$ Murdoch Children's Research Institute, Parkville, Australia

${ }^{10}$ PathWest Laboratory Medicine WA, Nedlands, Australia

${ }^{11}$ Genetic Health Service NZ, Wellington, New Zealand

${ }^{12}$ Centre for Clinical Genetics, Sydney Children's Hospital, Randwick, Australia

${ }^{13}$ Neurology, Sydney Children's Hospital, Randwick, Australia

${ }^{14}$ School of Biotechnology and Biomolecular Sciences, University of New South Wales, Randwick, Australia

${ }^{15}$ Clinical Genetics, Westmead Hospital, Westmead, Australia

${ }^{16}$ Genetic Medicine, The University of Sydney, Camperdown, Australia

${ }_{17}$ Starship Children's Health, Auckland District Health Board, Auckland, New Zealand

${ }_{18}$ Anatomic Pathology, Children's Hospital at Westmead, Westmead, Australia

${ }^{19}$ Cytogenetics, Children's Hospital at Westmead, Westmead, Australia

${ }^{20}$ Harvard Medical School, Boston, USA

Aim: To use patients with rare DMD intronic variants expressing varying levels of wild-type dystrophin to precisely correlate absolute levels of dystrophin with clinical phenotype. Methods: Exome, genome and/or RNA sequencing was performed and analyzed on six patients with possible dystrophinopathy. RT-PCR was performed to determine consequences for DMD pre-mRNA splicing. Quantitative western blot was used to determine levels of WT dystrophin, relative to control muscle. Results: Variants affecting DMD premRNA splicing were identified in six patients; intronic single nucleotide polymorphisms, to large, structural rearrangements. Deep analysis of patients with varying levels of normally-spliced and abnormally-spliced DMD mRNA, enabled quantification of levels of wild-type dystrophin, and correlation with clinical presentation. We define abrupt therapeutic range of dystrophin between 0-25\% levels of control muscle; $0-5 \%$ levels of wild-type dystrophin protein produce a DMD phenotype, whereas $25 \%$ levels result in myalgia without apparent weakness. Conclusions: RNA sequencing is instrumental in identifying splicing and structural variants (Cummings SciTransMed 2017). Nonsense-mediated mRNA decay is very efficient at removing enormous DMD mRNA which can confound RNA sequencing results. Non-quantitative amplification approaches provide invaluable information delineating the cDNA consequences of splicing and structural variants. In line with previous studies (Hoffman Neurology 1989), our study demonstrates the key range of dystrophin protein levels that influences clinical phenotype is 0-20\%. Differentiating our study - these levels are based on wild-type, not mutant dystrophin. Every percent of dystrophin present is vital for muscle function and better clinical outcome - this is an important implication for DMD genetic therapies. 


\section{THE EXPERIENCE OF AN ADULT CLINICAL GENOMICS UNIT - PAVING WAY TO PRECISION GENOMIC MEDICINE}

\author{
Devery $\mathrm{S}^{1}$, Dean $\mathrm{S}^{1}, \underline{\mathrm{Wu} \mathrm{K}}^{1,2,3,4}$ \\ ${ }^{1}$ St Vincent's Hospital Sydney, Darlinghurst, Australia \\ ${ }^{2}$ University of Sydney Discipline of Genetic Medicine, Sydney, Australia \\ ${ }^{3}$ UNSW School of Medicine, Sydney, Australia \\ ${ }^{4}$ Garvan Institute of Medical Research, Darlinghurst, Australia
}

The Clinical Genomics Unit (CGU) at St Vincent's Hospital Sydney was established in October 2016 to offer clinical, NATA-accredited, whole genome sequencing (WGS) as a mainstream diagnostic tool on a systematic basis, to public patients with suspected genetic disorders. Of 248 referrals received, 181 patients were seen. Of these, 137 patients have undergone testing, with 49 patients undergoing singleton-WGS with a combination of gene panel extracted from WGS, or WGS in its entirety. Results showed that when a panel of genes was extracted from WGS, a causative mutation was identified in $40 \%$ of cases (with variants of uncertain significance (VUS) in relevant genes identified in $20 \%$ of cases). This is opposed to a pickup rate of $17 \%$ when WGS was performed in its entirety (with $21 \%$ of VUSs found in relevant genes). By comparison, our pickup rate was $12 \%$ when traditional methods of single gene or targeted gene panels by exonic capture were applied. When WGS in its entirety was performed, incidental findings were noted in $13 \%$ of cases in our cohort. As of 2018, the CGU has started offering pharmacogenomics (PGx) testing on a routine basis, as a first step towards Precision Medicine Initiative on campus. The testing results are reviewed by a multidisciplinary team (MDT) and returned in a PGx MDT Clinic setting. We will present our model of care and the initial findings.

\section{COST-EFFECTIVENESS IN RETINOBLASTOMA IN RELATION TO REPRODUCTIVE TECHNOLOGIES AND PREIMPLANTATION GENETIC DIAGNOSIS (PGD)}

Zeppel $M^{\prime}$, Schofield $D^{\prime}$, Shrestha $R^{\prime}$, Staffieri $S^{2}$, Jamieson $R^{3}$, Jelovic $D^{3}$, Tan $O^{\prime}$

${ }^{1}$ Macquarie University, North Ryde, Australia

${ }^{2}$ Department of Ophthalmology, Royal Children's Hospital, Parkville, Australia

${ }^{3}$ Eye Genetics Research Unit, The Children's Hospital at Westmead, Children's

Medical Research Institute, Save Sight Institute, University of Sydney, Sydney, Australia

Background: Retinoblastoma $(\mathrm{Rb})$ is a pediatric cancer, leading to loss of vision, eye(s) or life. Approximately $40 \%$ of patients have a heritable form of the disease, caused by a mutation in the RB1 gene. Offspring of individuals with a heritable mutation are at a $50 \%$ risk of inheriting this mutation. Those that do require regular monitoring pre- and post-natally followed by aggressive and invasive treatments to control the tumors as they arise. Requiring numerous procedures under general anesthetic, treatment has a significant impact on the patient, family and hospital resources. Preimplantation genetic diagnosis (PGD) offers alternative reproductive choices for individuals with a heritable mutation. The costs of genomic sequencing are rapidly declining, while the availability of reproductive technologies is increasing. Thus, quantifying the cost-effectiveness of PGD in this context is increasingly important. Methods: We will model the cost of reproductive technology, number of affected/unaffected births, the quality of life gains and the incremental cost effectiveness (ICER) as cost per quality of life gained. Results: In a cohort of retinoblastoma survivors, we describe a cost-effectiveness study in relation to the use of reproductive technology. We compared the number of retinoblastoma survivors who have children with the general Australian population. We assess the quality of life gains and incremental cost-effectiveness ratio, taking into account the recurrence and cost of the use of in-vitro fertilization (IVF) and PGD. We also present the potential benefits of PGD for families with RB, and possible reasons why some families do not choose PGD.

\section{METABOLIC AND DIET DISEASE MANAGEMENT}

106. MANAGEMENT OF A TWIN PREGNANCY IN MAPLE SYRUP URINE DISEASE

Billmore $\mathrm{K}^{\prime}$, Tchan $\mathrm{M}^{\mathrm{l}}$

${ }^{1}$ Department of Genetic Medicine, Westmead Hospital, Westmead, Australia

The management of a twin pregnancy in a woman affected with maple syrup urine disease (MSUD) is described. The patient had classical disease diagnosed in the newborn period and had a successful singleton pregnancy eight years earlier. She was managed with a low protein diet and branch chain amino acid (BCAA) free Lamino acid supplements. Her BCAA concentrations were measured weekly and used to adjust her natural protein intake. We aimed to maintain her leucine levels below $300 \mu \mathrm{mol} / \mathrm{L}$ however this was not achieved until 18 weeks gestation. Consideration of the increased energy and protein requirements associated with twin gestations in the context of MSUD was necessary in this case. Adequate calorie intake was required to avoid metabolic decompensation and the use of L-amino acid mixtures, which are absorbed and oxidized more rapidly than intact protein sources, necessitated a higher total protein intake than standard twin gestations. We report our experience in managing this MSUD patient throughout the stages of her pregnancy.
107. WITHDRAWN

\section{BARRIERS TO EFFECTIVE PKU MANAGEMENT IN CARE FACILITIES}

Desai $A^{\prime}$, Rutledge $C^{\prime}$, Lefebure $K^{\prime}$, Fazio $T^{1,2}$, Panetta J', Fischer $C^{\prime}$, Quick $K^{\prime}$, De Jong $\mathrm{G}^{1,2}$

${ }^{I}$ Department of Medicine and Radiology, University of Melbourne, Parkville, Australia ${ }^{2}$ Royal Melbourne Hospital, Metabolic Diseases Unit, Melbourne, Australia

Background: Adults with previously untreated or late diagnosed phenylketonuria (PKU) can benefit from a low phenylalanine diet. Achieving dietary adherence and monitoring in patients living in care can be challenging, but rewarding. Aim: To describe the improvement of untreated PKU symptoms achieved due to dietary manipulation and to highlight the barriers of achieving good PKU control for untreated or late diagnosed PKU patients living in care. Methods: Over a 5-year period, the Metabolic Diseases Unit at the Royal Melbourne Hospital has initiated dietary therapy in 10 individuals with untreated PKU. Patients were commenced on low protein diets, with specialized PKU formula. Follow up was achieved through phone contact, Guthrie card reporting and outpatient reviews. Results: The improvement in untreated PKU behavior, assessed qualitatively on informal interview, has been positive for most of the individuals. Improvement in phenylalanine levels was achieved through addressing the barriers that exist for untreated patients. These include staffing, communication and adequate education. 9 out of 10 patients were noted by their carers to exhibit behavioral improvements such as decreased self-harm, increased attention and an improved ability to complete their personal activities of daily living. One patient ceased dietary intervention due to no perceived benefit. Conclusion: Adherence to a low protein diet with specialized PKU formula can improve symptoms in adults with previously undiagnosed or untreated PKU. When considering treatment, care should be taken to assess the living arrangements and the willingness of support staff to assist in dietary adherence. 


\section{A WEIGHT GAIN DILEMMA IN PKU}

Rutledge $C^{\prime}$, Desai $A^{\prime}$, Lefebure $K^{\prime}$, Panetta $J^{\prime}$, Fazio $T^{1,2}$, Quick $K^{\prime}$, Fischer $C^{\prime}$, de Jong $\mathrm{G}^{1,2}$

${ }^{1}$ Royal Melbourne Hospital, Metabolic Diseases Unit, Melbourne, Australia

${ }^{2}$ Department of Medicine and Radiology, University of Melbourne, Melbourne, Australia

Background: Obesity in the phenylketonuria (PKU) population occurs at similar rates to the general population. There are very few reports in the literature on the management of PKU patients undergoing bariatric procedures. We report the dietary management and monitoring of a 40 -year-old woman with a BMI of $54 \mathrm{~kg} / \mathrm{m} 2$ who underwent a sleeve gastrectomy. Aim: To describe the nutritional management and monitoring of a PKU patient prior to and post bariatric surgery. Methods: Weight loss attempts and counseling for maladaptive eating behavior over several years of engagement with the Metabolic Diseases Unit had been unsuccessful. The patient at her own initiative underwent a sleeve gastrectomy. Due to the high protein content of liquid VLCD preparations, prescription low caloric PKU formulas were requested to achieve pre-surgery weight loss and to assist with post op dietary management. Results: Significant weight loss (37\% of body weight) was achieved during the initial 8 months post-bariatric surgery. The catabolic state of the patient resulted in elevated Phenylalanine levels, 1104 um$\mathrm{mol} / \mathrm{L}$ at 4 weeks post op. Comparison of Phenylalanine levels reported on Guthrie card analysis in the 10-month post-surgical period showed Phenylalanine levels to average $756 \mathrm{umol} / \mathrm{L}$. Phenylalanine levels in the $12 \mathrm{months}$ pre surgery averaged $824 \mathrm{umol} / \mathrm{L}$. Conclusion: Phenylketonurics with refractory obesity can be successfully managed with bariatric surgery. Initial elevated phenylalanine levels due to catabolism were not longstanding, and with adequate dietary counseling and improved PKU formula intake were able to be reduced to pre-surgical levels.

\section{LATHOSTEROLOSIS: AN INBORN ERROR OF METABOLISM OF CHOLESTEROL BIOSYNTHESIS}

Hong $K^{\prime}$, Amor $D^{\prime}$, Hardikar $W^{2}$, Yaplito-Lee J3 , Eggington $M^{\prime}$, Mishra $A^{\prime}$, Pitt J'

${ }^{I}$ Victorian Clinical Genetics Services, Murdoch Children's Research Institute,

Melbourne, Australia

${ }^{2}$ Department of Gastroenterology and Clinical Nutrition, Royal Children's Hospital, Melbourne, Australia

${ }^{3}$ Department of Metabolic Medicine, Royal Children's Hospital, Melbourne, Australia

Lathosterolosis is a very rare disorder of cholesterol biosynthesis due to mutations in the SC5DL gene resulting in the deficiency of enzyme 3-beta-hydroxysteroid-delta-5-desaturase, causing a defect in conversion of lathosterol into 7-dehydrocholesterol. To date, only 4 patients have been described in the literature. This autosomal recessive condition lacks a distinctive clinical phenotype but is typically associated with congenital abnormalities of axial and appendicular skeleton, problems in liver, central nervous and urogenital systems. We report a patient who presented with developmental delay, bilateral cataracts and persistently abnormal liver function tests. Whole exome analysis confirmed the patient was compound heterozygous for missense (c.212A > T); p.(ASN71ILE) and nonsense (c.214C >T); p. (Gln72) mutations in the SC5DL gene which is consistent with lathosterolosis. This finding was further supported biochemically that showed an increased level of lathosterol (54 $\mu \mathrm{mol} / \mathrm{L}$; Range $<10$ ) with normal 7-dehydrocholesterol level. The patient was started on simvastatin which resulted in normalization of plasma lathosterol level $(8 \mu \mathrm{mol} / \mathrm{L})$ compared with baseline level $(30 \mu \mathrm{mol} / \mathrm{L})$. In addition, improvement in liver function tests and lipid profile was noted after 4 months. Statin treatment appears to be an effective treatment for lathosterolosis. More patients are required to better understand the variable phenotype associated with this disorder and the full effect of statin treatment.

\section{A BOY WITH SANFILIPPO SYNDROME WITH REFRACTORY HIP PAIN REQUIRING INTRA-THECAL OPOID INFUSION FOR CONTROL}

\author{
Inwood A ${ }^{1,5}, M^{2} N^{2}$, Mott $C^{3,4}$, Herbert $A^{3,4}$, Richards J ${ }^{4}$, McGill J', Alcock M ${ }^{4}$ \\ ${ }^{I}$ Queensland Lifespan Metabolic Medicine Service, Lady Cilento Children's Hospital, \\ South Brisbane, Australia \\ ${ }^{2}$ Department of Neurosurgery, Lady Cilento Children's Hospital, South Brisbane, \\ Australia \\ ${ }^{3}$ Queensland Paediatric Palliative Care Pain Service, Lady Cilento Children's \\ Hospital, South Brisbane, Australia \\ ${ }^{4}$ Queensland Paediatric Pain Service, Lady Cilento Children's Hospital, South \\ Brisbane, Australia \\ ${ }_{5}^{5}$ University of Queensland, St Lucia, Brisbane, Australia
}

A 7-year-old male with known mucopolysaccharidosis IIIA (MPSIIIA) presented with hip pain. MRI identified bilateral avascular necrosis of the hips. Pain was initially managed successfully with intra-articular steroid injections and 1 to 3-monthly Pamidronate infusions for 4.5 years. At 11.8 years, the pain became resistant to ongoing Pamidronate infusions and multimodal analgesia. Oral medications trialed included paracetamol, NSAIDs (ibuprofen and meloxicam), long and short acting opioids (oxycodone/naloxone sustained release, morphine sulphate sustained release, methadone), gabapentin, antidepressants, clonidine, ketamine and baclofen. Parenteral analgesia trialed included fentanyl patches and subcutaneous ketamine infusions. At 12.8 years, a right-sided femoral head excision and valgus femoral osteotomy was performed, but severe pain persisted. Nurse/carer controlled analgesia of intravenous hydromorphone provided modest benefit. A tunneled femoral nerve catheter and continuous bupivacaine infusion was trialed for one week but was ineffective. At 13 years, a test dose of intrathecal bupivacaine and morphine provided temporary analgesia. At 13.2 years, an intrathecal Port-a-cath was implanted to provide a continuous infusion of morphine and bupivacaine. This allowed the child to remain pain free, except during a brief period due to inadvertent port decannulation. Due to skin integrity problems, the port was deneedled and pain had not returned for 12 weeks. Conclusion: Bone pain in MPSIIIA can be difficult to manage. When systemic multimodal analgesia and peripheral regional analgesia are ineffective and pain remains localized, continuous intrathecal analgesia can be considered. The intrathecal route avoided adverse effects seen with systemic opioids and was very effective in this case.

\section{NURSES WORKING TO TOP OF SCOPE OF PRACTICE CAN REDUCE CLINICAL WORKLOAD IN METABOLIC SERVICES}

Inwood $A^{1,4}, \underline{\text { Smith } S^{\prime}}$, Roper $T^{\prime}$, Spicer J', Lipke $M^{\prime}$, Coman $D^{1,5}$, Kynaston J', McGill J,3

${ }_{2}^{1}$ Queensland Lifespan Metabolic Medicine Service, South Brisbane, Australia 2 Department of General Paediatrics, Lady Cilento Children's Hospital, South Brisbane, Australia

${ }^{3}$ Pathology Queensland, Royal Brisbane Women's Hospital, Herston, Australia ${ }^{4}$ School of Midwifery and Nursing, University of Queensland, St Lucia, Brisbane, Australia

${ }^{5}$ School of Medicine, University of Queensland, St Lucia, Brisbane, Australia

Background: Since the introduction of a Nurse Practitioner (NP) role in the Queensland Metabolic Medicine Service, the number of emergency department (ED) consults and re-presentations for children with hypoglycemia has significantly declined. Methods: A retrospective chart audit revealed that in the year of 2017, 53 children presented with hypoglycemia associated with an inter-current illness that affected appetite. Most children fasted the equivalent of more than 30 hours without a solid source of carbohydrate. Results: Fifteen percent re-presented to the ED with 1 patient representing twice prior to attending the NP clinic with zero re-presentations after attending the NP clinic. Discussion: A hospital-wide policy was created outlining appropriate investigations at time of hypoglycemia, management in the acute setting and if required, admission under the 
general pediatric team with a post discharge referral to the NP clinic. The NP clinic included review of the hypoglycemia screen to exclude the known endocrine and metabolic causes of hypoglycemia in this age group, leaving a likely diagnosis of ketotic hypoglycemia of childhood; pathophysiology of glucose metabolism, age appropriate carbohydrate sick day fluid plan with recipes; instructions when to seek medical review. Conclusion: Parents/carers respond to education outside of the critical time of illness. Education regarding sick day management can reduce the high dependence on subspecialty services which allows clinician time to be allocated toward acutely presenting metabolic disorders.

\section{REDUCING PLASMA LEUCINE IN THE ACUTE SITUATION OF CLASSICAL MSUD: CLINICAL AUDIT}

Mullane $E^{\prime}$, Fitzell $K^{\prime}$, Evans $M^{\prime}$

${ }^{1}$ Royal Children's Hospital Melbourne, Parkville, Australia

Aim: To compare time to reduce plasma leucine (PL) in patients with MSUD on dietary manipulation alone, versus dietary manipulation plus hemofiltration. Method: A retrospective audit of patients diagnosed with classical MSUD since $2009(n=8)$ was completed. Plasma leucine (PL), energy and synthetic amino acid (AA) intakes were compared for episodes when PL was $>800 \mathrm{umol} / 1$. $R e$ sults: Data were analyzed for 28 episodes to treat high PL $(n=8$ NDx, $n=20$ subsequent admissions). Overall, $5 / 8$ patients required hemofiltration with $3 / 8$ at initial neonatal diagnosis (NDx). Median PL at NDx $(n=8)$ was $2268 \mu \mathrm{mol} / 1$ (range: $1850-3589)$. For all subsequent admissions $(n=20)$, median PL was $1052 \mu \mathrm{mol} / 1$ (range: $822-1572$ ). Time to achieve plasma leucine $<300$ at NDx $(n=8)$ was median 64.1 hours (range: $33.5-180.5)$. For subsequent admissions $(n=20)$, median 44.8 hours (range: $24-129.8)$. For all episodes combined $(n=28)$; median 54.3 hours (range: $24-180)$. For $5 / 8$ patients requiring hemofiltration during admission, median time to attain PL $<300 \mu \mathrm{mol} / 1$ was median 54.3 hours (range: $28.5-$ 110). For patients on diet only median 53.9 hours (range: $24-180$ ). Patients achieved their recommended energy intake in median 8 hours (range: 1-18.8). Time to achieve AA target varied depending on route of nutrition support, age and gastrointestinal symptoms. Conclusion: Time taken to reduce PL was similar between patients with or without hemofiltration. Time to reduce PL and achieve AA targets was greater at NDx than subsequent admissions. Achieving nutritional guidelines is challenging in neonates and patients with enteral feeding intolerance and may increase the time to reduce PL adequately in patients not hemofiltered.

\section{GASTROINTESTINAL SYMPTOMS IN FABRY DISEASE: RESPONSE TO THERAPY}

Nedanovski B', Talbot $A^{\prime}$, Nicholls $K^{1,2}$

${ }^{I}$ The Royal Melbourne Hospital Department of Nephrology, Melbourne, Australia

${ }^{2}$ The University of Melbourne, Melbourne, Australia

Background: Gastrointestinal (GI) symptoms significantly reduce the quality of life in patients with Fabry disease. The current approved treatment is enzyme replacement therapy (ERT). Our understanding of ERT and its effects specifically on the GI manifestations is limited. Aims: (1) Characterise the GI symptoms of our patients with Fabry disease. (2) Determine the effects of enzyme replacement therapy. Methods: The patients in the population studied have all been diagnosed with Fabry disease. GI symptom data was collected at each routine visit. This was done through a questionnaire as well as a bowel diary encompassing the month preceding the visit. The presence of diarrhoea was defined as $>3$ stool samples per day and/or 'diarrhoea' noted in the comments section. Significance testing was performed with Fischer's exact test or one-way ANOVA depending on the data. Results: Questionnaires from 101 patients (38 males, 63 females) were analyzed at baseline. $47.5 \%$ of patients reported either abdominal pain or diarrhoea (males $63.2 \%$ vs females $38.1 \%, p=.023$ ). Within a month, diarrhoeal symptoms were present on average in $18.6 \%$ of days (males $26.4 \%$ vs females $11.3 \%, p=.01$ ). $14 \%$ of the patients experienced diarrhoea more days than not. 19 patients had been undergoing ERT for approximately 10 years, 9 had diarrhoeal symptoms at baseline. Symptom severity improved post one year of treatment $(p<.01)$. This continued to improve 11-13 years post the commencement of therapy $(p<.001)$. Conclusion: The data presented here quantifies the diarrhoeal symptoms of our Fabry disease cohort and suggests that ERT improves symptoms.

\section{DIETARY MANAGEMENT OF CLASSICAL HOMOCYSTINURIA IN INFANCY - CASE STUDY REPORT}

Paramore $C^{1}$, Thompson $S^{1}$, Ellaway $C^{1,2}$

${ }^{I}$ Sydney Children's Hospital Network, Westmead, Australia

${ }^{2}$ University of Sydney, Sydney, Australia

Classical homocystinuria (HCU), due to cystathionine beta synthase deficiency, affects the metabolism of methionine. Dietary management includes protein restriction, supplemented with methioninefree amino acid medical foods (AAM) if appropriate. Some patients are responsive to pyridoxine but newborn screening is less likely to detect this group. Four patients with HCU have been diagnosed following a positive newborn screen in NSW, and two other infants through sibling early diagnosis. The first three NBS positive patients were treated by reduction in standard formula or breastmilk and supplementation with AAM, adjusting proportions depending on plasma total homocysteine (THcY) levels. Initial THcy levels of 261, 130 and 193 took 17, 27 and 56 days to decrease to $<100 \mathrm{umol} / \mathrm{l}$ on dietary treatment. The 6 th and most recently diagnosed patient (now aged 6 weeks) has been managed by initial cessation of standard formula and replacement with AAM as per BIMDG guidelines (BIMDG, 2015). Diet was commenced on day 23 of age with 3 days on AAM alone. On day 26 feeds were changed to standard formula + AAM providing natural protein intake of $1.8 \mathrm{~g} / \mathrm{kg}$. Methionine and THcY levels of $1188 \mathrm{umol} / \mathrm{l}$ (normal range 14-47) and 240 umol/l (normal range 3.3-19.2) respectively on day 22 decreased to 63 and 105 on day 36. Pyridoxine (50 $\mathrm{mg} /$ day) was not trialled alone but responsiveness will be reassessed once THcY levels have stablilized. The new protocol decreased time taken for toxic metabolites to reduce to within treatment recommendations. This may have implications for AAM tolerance. http://www.bimdg.org.uk/site/guidelines-enbs.asp?t=3

\section{MOLECULAR GENETICS}

\section{GENOTYPING OF BETA-THALASSEMIA USING LOOP-MEDIATED ISOTHERMAL AMPLIFICATION (LAMP) IN BANGLADESH}

Sarker $S^{\prime}$, Islam $A^{\prime}$, Akhteruzzaman $S^{\prime}$, Begum $N^{\prime}$, Islam $M^{\prime}$, Sajib $A^{\prime}$, Sultana $N^{\prime}$, Qadri S', Qadri F', Mannoor K'

${ }^{I}$ University Of Dhaka, Bangladesh

Background: $\beta$-thalassemia and $\mathrm{Hb}$-E disease is commonly present among many populations worldwide. Current available Hb-E diagnostic techniques require advanced laboratory equipment and are not cost effective. Objective: The aim of the current study is to develop an easy to use, rapid and cost effective method for the diagnosis of beta-thalassemia. Methods: Initially, we have collected 160 transfusion dependent beta-thalassemia patients' blood specimens to determine the most common mutation among Bangladeshi population. We have designed isothermal loop-mediated isothermal amplification (LAMP) based mutation detection technique to identify the specific mutation from the patients' blood specimen 
which comprises following steps: (1) extracting human DNA from blood; (2) performing LAMP amplification; and (3) visualization of LAMP amplification to identify the specific mutation of betaglobin gene. Results: We have sequenced the $428 \mathrm{bp}$ long stretch of HBB gene encompassing the mutational hot-spot region (exon 1 , intron 1 and a portion of exon 2) of beta-globin gene. We have identified IVS1 -5 (G > C): 47\%, CD $26(\mathrm{G}>\mathrm{A}) / \mathrm{HbE}: 42 \%, \mathrm{Cd} 41 / 42$ (-TTCT): $3 \%$, IVS $1-1(\mathrm{G}>\mathrm{T}): 1 \%$ as the most common mutation in Bangladesh using Sanger's DNA sequencing of beta-globin gene. Based on this data, we have designed LAMP primers for genotyping of thalassemia. Specificity and sensitivity of the method will be analyzed using patient's blood specimen. Conclusion: Successful implementation of this technique will be cost effective as well as eliminate the necessity of any sophisticated instrument and expert laboratory personnel.

\section{DIAGNOSTIC YIELD OF ARRAY CGH IN VIETNAMESE CHILDREN WITH AUTISM SPECTRUM DISORDER}

Bui Thi Phuong $\mathrm{H}^{\prime, 2}$, Do Huy $\mathrm{D}^{\prime}$, Ly Thi Thanh $\mathrm{H}^{\prime}$, Nguyen Trung $\mathrm{K}^{\prime}$, Tran Trung $\mathrm{K}^{\prime}$, Le Sy $\mathrm{V}^{\prime}$, Mukhopadhyay $\mathrm{A}^{2}$, Nguyen Thanh $\mathrm{L}^{\prime}$

${ }^{I}$ Vinmec Research Institute of Stem Cell and Gene Technology, Viet Nam

${ }^{2}$ University of Salford, Manchester, United Kingdom

Background: Autism spectrum disorder (ASD) is a developmental disorder with a prevalence of around $1 \%$ children worldwide and characterized by the effects of children behavior, communication, social interaction, and personal development. Currently, chromosomal microarray is considered as the first-tier test for autistic children with diagnostic yield of around $10 \%$. However, this advanced test is limited in clinical studies and autistic diagnoses in Vietnam. Aim: Hence, the aim of this study is to identify genetic patterns of ASD in the Vietnamese children by using the Microarray-based Comparative Genomic Hybridization (aCGH). Methods: One hundred trios whose children with ASD detected using the Diagnostic and Statistical Manual V (DSM-V), Autism Diagnostic Observation Schedule (ADOS), and Childhood Autism Rating Scale (CARS) have been recruited at Vinmec International Hospital in Hanoi, Vietnam. The RETT and Fragile X tests were performed to exclude probands with the related syndromes. The aCGH test was performed on probands and their parents to detect copy number variants (CNVs). Results: We identified 16 de-novo CNVs (7 gain and 9 loss CNVs) containing clinically significant genes in 15 patients ( 3 females and 12 males). Conclusion: The diagnostic yield of $15 \%$ demonstrates the efficiency of the chromosomal microarray as the first-tier test for autistic children in Vietnam.

\section{CHROMOSOMAL MICROARRAY IN POSTMORTEM} FETAL SAMPLES

Cham $B^{\prime}$, Lian $D^{\prime}$, Law $H^{\prime}$, Yong $M^{\prime}$, Tan $Y^{\prime}$, Yeo $S^{\prime}$, Lai $A^{\prime}$

${ }^{I}$ KK Women's and Children's Hospital, Singapore

Aim: The objective of this study was to evaluate the diagnostic yield of chromosomal microarray (CMA) analysis in fetal postmortem samples. Methods: This was a retrospective study of postmortem fetal samples received by a single centre who underwent CMA testing from July 2014 to March 2018. Indications for postmortem included fetuses with ultrasound anomalies that resulted in pregnancy termination and intrauterine death. Samples were run on the Agilent $4 \times 180 \mathrm{k}+$ SNP array. Results: Of the 31 fetal samples received, 7 (23\%) had abnormal CMA results -6 were classified as pathogenic, 1 was a variant of uncertain significance. CMA failed to yield results in 1 sample due to poor sample quality. The most frequently observed ultrasound anomalies were skeletal (32\%, $n=10)$, cardiovascular $(23 \%, n=7)$ and central nervous system $(19 \%, n=6)$. Discussion: Chromosomal microarray analysis results showed concordance with conventional karyotyping results in our series. However, CMA was able to provide a result more often than karyotyping ( $96.7 \%$ vs $70.4 \%$ ), as CMA does not require live cells, thus analysis can be done on samples that would otherwise not be suitable for karyotype analysis. Three of the seven abnormal results were unlikely to have been detected via karyotyping and represents a potentially increased diagnostic yield. Reaching a genetic diagnosis provides clinicians with information for recurrence-risk counseling and provides closure for families. Further genetic studies (e.g., testing for single gene disorders, whole exome sequencing) following a normal CMA result may increase the diagnostic yield in this population.

\section{GENE DISCOVERY IN A SMALL MOTOR NEURON DISEASE KINDRED USING COMPUTATIONAL AND FUNCTIONAL ANALYSIS PIPELINES}

Chan Moi Fat $S^{1}$, Fifita J', McCann $E^{1}$, Williams $K^{1}$, Twine $N^{1,2}$, Bauer $D^{2}$, Nicholson $\mathrm{G}^{1,3}$, Blair I'

${ }^{I}$ Centre for MND Research, Department of Biomedical Sciences, Faculty of Medicine and Health Sciences, Macquarie University, Sydney, New South Wales, Australia ${ }^{2}$ Commonwealth Scientific and Industrial Research Organization, Health \& Biosecurity Flagship, Sydney, New South Wales, Australia

${ }_{3}^{3}$ ANZAC Research Institute, University of Sydney, Concord Hospital, Sydney, New South Wales, Australia

Motor neuron disease (MND) is a fatal neurodegenerative disease, caused by the degeneration of upper and lower motor neurons. To date, gene mutations are the only proven cause. Approximately $10 \%$ of cases are hereditary (familial), of which one third still have an unknown genetic cause. This study aims to identify the causative gene mutation in a small MND kindred using a combination of next generation sequencing data, in silico and in vitro tools. Whole exome sequencing (WES) and whole genome sequencing (WGS) data were generated from two affected siblings and their 'married-in' parent. Shared variant analysis of WES data, including filtering to nonsynonymous and novel variants, led to a total of 21 candidate variants. Additional filtering from and age- and population-matched control databases reduced this list to 18 candidate variants, all validated by Sanger sequencing. In silico analyses, namely protein prediction programs, conservation, genic tolerance, expression in brain/spinal cord, and gene function were used to rank each candidate variant. The top three high priority variants were then selected for a novel in vitro analysis pipeline to undergo functional assessment. Additionally, these individuals also underwent WGS, and shared variant analysis is underway to validate candidates and identify additional non-coding candidate variants. We demonstrate that the combination of our genetic and custom in silico and in vitro pipelines has potential to elucidate the remaining causative gene mutations in small MND kindreds. This is vital to improve our understanding of disease biology and aid in the development of diagnostics and future treatments.

\section{20 YEARS OF NOTCH3 SEQUENCING FOR CADASIL: A} LABORATORY PERSPECTIVE OF DIAGNOSTIC RATES

Dunn $\mathrm{P}^{1,2}$, Maksemous $\mathrm{N}^{1,2}$, Smith $\mathrm{R}^{1,2}$, Sutherland $\mathrm{H}^{1,2}$, Haupt $\mathrm{L}^{1,2}$, Griffiths $\mathrm{L}^{1,2}$

${ }_{1}^{1}$ Queensland University of Technology, Kelvin Grove, Australia

2 Institute of Health and Biomedical Innovation, Kelvin Grove, Australia

Background: Cerebral autosomal dominant arteriopathy with subcortical infarcts and leukoencephalopathy (CADASIL) is the most common monogenic cause of familial stroke in the world. To confirm the diagnosis of CADASIL, it is reliant on the histological observation of granular osmiophilic material (GOM) in tissue biopsies or through the identification of mutations in NOTCH3. The Genomics Research Centre has been undertaking diagnostic testing for NOTCH3 mutations associated with CADASIL since 1997. Work originally utilized Sanger sequencing that targeted specific exons. More recently next generation sequencing (NGS) technologies such as the GRC 5-gene custom panel and whole exome 
sequencing (WES) have been used. Methods: Data from 700 patient samples was analyzed for 756 tests utilising three different sequencing technologies, Sanger sequencing accounted for 443 tests, our 5-gene NGS gene panel accounted for 310 tests and targeted WES was performed for 3 tests. Results: In total $15 \%$ of patient samples $(n=105 / 700)$ were identified to have a mutation. Testing found disease causing mutations in $12.6 \%(n=56 / 443)$ tests using Sanger sequencing, $15.4 \%(n=48 / 310)$ via the GRC 5-gene panel and $33.3 \%(n=1 / 3)$ through WES. Further analysis of the work stratified the number of mutations based on the number of exons, level of pathogenicity and the classification of mutations as known or novel. Conclusion: A systematic review of NOTCH3 mutation testing data from 1997 to 2017 identified the diagnostic rate of pathogenic findings within our cohort and determined the GRC 5-gene panel increases our ability to identify disease causing mutations in NOTCH3.

\section{A NOVEL FINDING WITHIN THE FMR1 GENE PROMOTER IN A MALE INFANT}

Elliott $E^{\prime}$, Fazeli $C^{\prime}$, Fenn $M^{2}$, Angell $E^{1}$, Bickley $L^{3}$, Lewis $R^{1}$, Taylor $P^{3}$, Chia $N^{1,2}$

${ }^{1}$ QML Pathology, Murarrie, Brisbane, Australia

${ }^{2}$ Western Diagnostic Pathology, Myaree, Australia

${ }_{3}^{3}$ Genomic Diagnostics, Heidelberg, Australia

Background: Fragile X Syndrome (FXS) is an X-linked hereditary disorder associated with intellectual and developmental disabilities. FXS is caused by expansion of an unstable CGG trinucleotide repeat in the promoter region of the Fragile Mental Retardation 1 gene (FMR1) on Xq27.3 (NM_002024.4). Expansions greater than 200 repeats result in DNA hypermethylation of the FMR1 promoter and absent expression of FMRP. Aim: To investigate the origin of two FMR1 alleles of 30 and 40 CGG repeats detected in a karyotypically 46, XY two year old infant with global developmental delay. Methodology: To determine the CGG repeat number, DNA was tested by PCR followed by fragment analysis, then confirmed using AmplideX FMR1 (IVD CE) PCR kit. Two additional sets of PCR primers flanking the promoter region were designed. The PCR products were purified separately and Sanger sequenced. Microarray analysis and karyotyping were performed on both the patient and parents. Results: FMR1 PCR analysis detected 31 CGG repeats (paternal), 20 and 30 repeats (maternal) which confirmed maternal inheritance of 30 repeats in the proband. No abnormality was detected by cytogenetic and microarray analysis. The custom designed primer pairs detected a 30bp duplicated sequence in the GC rich region (10 CGG repeats). Sequence analysis of both PCR products showed $100 \%$ homology to the FMR1 promoter region. Conclusion: Our study demonstrated that the 40 CGG repeat was derived from a mosaic duplication in the FMR1 promoter region. The presence of two alleles in the normal reference range is a novel finding in a male patient.

\section{ENZYMOLOGY AND ANALYTE MEASUREMENT FOR THE LSDS ARE NOT DEAD}

Ellul $M^{\prime}$, Chin $S^{\prime}$, Brion $K^{\prime}$, Pyragius $T^{\prime}$, Fuller $M^{\prime, 2}$, Fletcher J'

${ }^{I}$ Genetics and Molecular Pathology, SA Pathology, Adelaide, Australia

${ }^{2}$ School of Medicine, University of Adelaide, Adelaide, Australia

Introduction: The diagnosis of lysosomal storage disorders traditionally results from the detection of reduced enzyme activities. However, in the genomic age, molecular diagnosis is increasingly used for diagnosis. Detection of two likely pathogenic or pathogenic variants is sufficient for a diagnosis, but variants of unknown significance may raise more questions than they answer. Methods: Request forms sent to our laboratory for confirmatory testing were extracted from the laboratory information systems. Test results were reviewed for these patients and classified into: diagnosis confirmed, diagnosis excluded and result undetermined. Results: Over the past 2 years, we have been referred 7 samples to confirm diagnoses made by molecular methods (Metachromatic leukodystrophy, Krabbe, MPS II, MPS IIIA, MPS IIIC, MPS VII, and CDG1A). Of these, the diagnosis was confirmed in four patients and excluded in two patients. In one patient, the diagnosis remained uncertain. Conclusion: Variants of unknown significance and pseudodeficiencies are increasingly being found in lysosomal storage disorders. Enzymology remains the mainstay of diagnostic confirmation for novel variants. Analyte measurements may be required to confirm the effect of multiple pseudodeficiencies on enzyme activity.

\section{SIMULTANEOUS EXON AND WHOLE-GENOME RESOLUTION COPY NUMBER ANALYSIS FOR CANCER DIAGNOSIS}

Fellowes $A^{\prime}$, Markham $J^{\prime}$, Legaie $R^{\prime}$, Yerneni $S^{\prime}$, Lupat $R^{\prime}$, De Silva $W^{\prime}$, Ryland $\mathrm{G}^{\prime}$, Blombery $\mathrm{P}^{\mathrm{l}}$

${ }^{\prime}$ Peter MacCallum Cancer Centre, Melbourne, Australia

Somatic copy number alteration (SCNA), arising from defects in replication, segregation or repair, is a conspicuous feature of almost all cancer genomes. In addition, germline copy number mutations, resulting from exon and gene scale deletion of tumor suppressor genes are significant causes of inherited cancer susceptibility. Both germline and somatic copy number changes have diagnostic and therapeutic utility but are cumbersome to detect using traditional diagnostic tools. In this study we assessed the accuracy and clinical utility of copy number estimates derived from targeted next generation sequencing (NGS) analysis, by comparison to those obtained from gold standard copy number methods. We used a novel read counting and visualization tool (GAFFA) to assess copy number changes derived from targeted NGS analysis of standard reference materials, clinical formalin-fixed paraffin embedded (FFPE) solid tumors, hematological malignancies, and germline samples. Comparator methods included droplet digital PCR (ddPCR), multiplex ligation-dependant probe amplification (MLPA), fluorescent in situ hybridization (FISH), immunohistochemistry (IHC) and microarray. Using reference cell lines containing focal gene amplifications, NGS derived copy number was highly correlated with ddPCR $\left(r^{2}=.9956\right)$. On a series of 43 samples containing MLPA verified germline copy number mutations, we saw $100 \%$ positive percent agreement (PPA) and $100 \%$ positive predictive value (PPV). Actionable numerical and structural SCNA observed in clinical FFPE and hematological samples were verified by FISH, IHC and microarray with high concordance. Accurate copy number estimation can be derived from NGS analysis of a range of clinical sample types and provides diagnostic and therapuetic information without additional analytical procedures.

\section{WITHDRAWN}

\section{HEREDITARY AMYLOIDOSIS}

Fisk $K^{1}$, Hertzog $A^{1,2}$, Booth $D^{3}$, Taylor $M^{4}$, Stewart $G^{4}$, Bennetts $B^{1,5}$

${ }^{1}$ Sydney Genome Diagnostics, Western Sydney Genetic Program, The Childrens Hospital at Westmead, Sydney, Australia

${ }^{2}$ NSW Biochemical Genetics service, Western Sydney Genetic Program, The Childrens Hospital at Westmead, Sydney, Australia

${ }^{3}$ Westmead Institute for Medical Research, University of Sydney, Sydney, Australia

${ }^{4}$ Department of Clinical Immunology and Allergy, Westmead Hospital, Sydney Medical School, University of Sydney, Sydney, Australia

${ }_{5}^{5}$ Disciplines of Genetic Medicine and Paediatrics and Child Health, The University of Sydney, Sydney, Australia

Introduction: Hereditary amyloidosis is an autosomal dominant disorder of abnormal protein folding, that progressively disrupts tissue structures and causes disease. Variants have been identified in multiple genes to be causative of hereditary amyloidosis, however, the most frequently occurring variants have been identified in the TTR, FGA, LYZ and APOA1 genes. Method: A cohort of patients referred 
for '?hereditary amyloidosis' have been screened in our laboratory for TTR exons 1-4, FGA codons p.510 to p.630 in exon 5, APOA1 exons 3 and 4 and LYZ exons 2 and 4, using Sanger sequencing. These regions cover $94 \%$ of known pathogenic hereditary amyloidosis variants currently reported in the amyloidosis mutation database. Targeted whole gene screening has also been performed where the constituent amyloid protein has been identified by proteomics. $R e$ sults: Both previously reported pathogenic variants, as well as novel variants of uncertain clinical significance (VOUS), have been identified in the referred cohort. Classification of novel variants is difficult due to the minimal literature, the unknown consequences on protein folding and the potential for incomplete penetrance or variable phenotypes within a family. Conclusion: We have established a diagnostic service, screening the genes/regions most commonly associated with hereditary amyloidosis. Determination of the cause of amyloidosis is important for treatment, outcomes as well as familial testing. It is also necessary to rule out immunoglobulin light chain (AL) amyloidosis before a diagnosis of hereditary amyloidosis can be made in order to avoid unnecessary treatments. Our findings should also help to expand the current literature on hereditary amyloidosis.

\section{IMPROVED, ACMG-COMPLIANT, IN SILICO PREDICTION OF PATHOGENICITY FOR P53 MISSENSE VARIANTS}

\author{
Fortuno $C^{1}$, James $P^{2}$, Young $E^{3}$, Feng $B^{4}$, Olivier $M^{5}$, Pesaran $T^{6}$, Tavtigian $S^{3}$, \\ Spurdle $A^{1}$ \\ ${ }^{1}$ QIMR Berghofer Medical Research Institute, Genetics and Computational Division, \\ Herston, Australia \\ ${ }^{2}$ Parkville Familial Cancer Centre, Peter MacCallum Cancer Centre and Royal \\ Melbourne Hospital, Melbourne, Australia \\ ${ }^{3}$ Department of Oncological Sciences and Huntsman Cancer Institute, University of \\ Utah School of Medicine, Salt Lake City, United States \\ ${ }^{4}$ Department of Dermatology and Huntsman Cancer Institute, University of Utah \\ School of Medicine, Salt Lake City, United States \\ ${ }^{5}$ Molecular Mechanisms and Biomarkers Group, International Agency for Research on \\ Cancer, Lyon, France \\ ${ }^{6}$ Ambry Genetics, Viejo, United States
}

Clinical interpretation of germline missense variants represents a major challenge, including those in the TP53 Li-Fraumeni syndrome gene. Bioinformatic prediction is a key part of variant classification strategies. We aimed to optimize the performance of the AlignGVGD tool used for p53 missense variant prediction, and compare its performance to other bioinformatic tools (SIFT, PolyPhen2) and ensemble methods (REVEL, BayesDel). Reference sets of assumed pathogenic and assumed benign variants were defined using functional and/or clinical evidence. Area under the curve and Matthews correlation coefficient (MCC) values were used as objective functions to select an optimized protein multi-sequence alignment with best performance for Align-GVGD. MCC comparison of tools using binary categories, alone and in independent combinations, showed optimized Align-GVGD (C15 cut-off) combined with BayesDel ( 0.16 cut-off), or with REVEL ( 0.5 cut-off), to have the best overall performance. Further, a semi-quantitative approach using multiple tiers of bioinformatic predictions, validated using an independent set of non-functional and functional variants, supported use of Align-GVGD and BayesDel prediction for different strength of evidence levels in ACMG/AMP variant classification guidelines. Here, we provide rationale for bioinformatic tool selection for $\mathrm{p} 53$ missense variant classification, and have computed predictions for every possible missense variant to facilitate its use. Finally, we also provide insights into a quantitative method by combining the outputs of selected bioinformatics tools, transformed into likelihood ratios of pathogenicity, with a novel approach known as somatic to germline ratio.

\section{EXPANDING THE PHENOTYPE OF INTELLECTUAL DISABILITY CAUSED BY HIVEP2 VARIANTS}

\author{
Goldsmith $\mathrm{H}^{\prime}$, Goel $\mathrm{H}^{1,2}$, Williams $\mathrm{M}^{3,4,5}$ \\ ${ }^{1}$ Hunter Genetics, Hunter New England Local Health District, Newcastle, Australia \\ ${ }^{2}$ University of Newcastle, Callaghan, Australia \\ ${ }^{3}$ Mater Research Institute, The University of Queensland, Woolloongabba, Australia \\ ${ }^{4}$ Faculty of Medicine, The University of Queensland, St Lucia, Australia \\ ${ }^{5}$ Genetic Pathology, Mater Pathology, South Brisbane, Australia
}

Background: The human immunodeficiency virus enhancer binding protein 2 (HIVEP2) gene encodes a large transcription factor; HIVEP2, which is expressed predominantly in the brain and has implications in neurodevelopment. De novo pathogenic variants in HIVEP2 have previously been published in nine patients with intellectual disability and various dysmorphic features. We describe two additional patients with intellectual disability and dysmorphic features who have previously unreported features, including hyperphagia and Angelman-like behaviors. Aim: To report on the phenotype and genotype of patients with de novo pathogenic HIVEP2 variants, and to identify if loss-of-function variants that escape nonsensemediated decay result in less severe phenotype. Methods: Whole exome sequencing was utilized in the investigation of the patients who had previously incurred a rigorous genetic workup for their neurodevelopmental delay, and in whom no genetic cause was detected. Information pertaining to phenotype and genotype for new patients was collated along with data from previous reports. $R e$ sults: Patients with HIVEP2 variants have a broad clinical spectrum, with features including intellectual disability, limited speech and hypotonia. Dysmorphic features vary between patients. No clear association between the type of gene aberration and phenotype can yet be concluded. Conclusion: The addition of these two patients to the previously documented patients with de novo pathogenic HIVEP2 variants suggests that the phenotypic spectrum is broader then first noted. Whole exome sequencing needs to be considered in the genetic diagnostic armamentarium for patients with intellectual disability of inconclusive etiology.

\section{NEW GENERATION DIAGNOSTICS FOR SOMATIC HYPERMUTATION OF THE IGHV GENE IN CLL PROGNOSTICATION}

$\underline{\text { Hall R' }}$, Burdett J', Kuss B', Grist $S^{\prime}$

${ }^{I}$ SA Pathology, Bedford Park, Australia

Background: Somatic hypermutation (SHM) of the immunoglobulin heavy chain variable (IgHV) gene sequence is a strong indicator of prognosis in Chronic Lymphocytic Leukemia (CLL) patients. Traditional SHM studies analyze the dominant clone present in the patient by amplification and Sanger sequencing. When a single productive rearrangement is detected, analysis of mutation status and interpretation is simple. However, the frequent presence of additional rearrangements presents difficulties for the laboratory, with up to $30 \%$ of cases unresolvable by standard Sanger techniques. Next Generation Sequencing offers significant improvements to SHM analysis in CLL as the methodology allows all unique sequences present to be simultaneously sequenced then demultiplexed into useable data, a dramatic improvement over the current standard of Sanger sequencing. Methods: A cohort of 716 patients with CLL were assessed for IgHV clonality using the LymphoTrack ${ }^{\circledR}$ Dx IGH FR1 Assay - MiSeq ${ }^{\circledR}$ from Invivoscribe. Analysis of mutation frequency and gene usage was determined using the IMGT/V-Quest database and subset analysis was performed using the ARResT/AssignSubsets tool. Results were compared to 333 patients previously studied by Sanger methods. Results: $42 \%$ of patients in the NGS cohort were assessed as hypermutated, 50\% were assessed to be not hypermutated, $6 \%$ were borderline and $<2 \%$ failed to analyze as compared to $30 \%$ failure to analyze in the Sanger cohort. Conclusion: NGS for 
SHM in CLL is a significant improvement over traditional Sanger sequencing methods in which $30 \%$ of patients failed to produce useable data due to the presence of multiple rearrangements that were unable to be deconvoluted.

\section{SO YOU HAVE A .VCF FILE WITH A LOT OF VARIANTS - NOW WHAT?}

Hall R', Grist $\mathrm{S}^{\prime}$

${ }^{1}$ SA Pathology, Bedford Park, Australia

Introduction: As Next Generation Sequencing becomes commonplace in clinical practise, the number of novel variants identified increases exponentially. Large numbers of these variants require downstream analyses to evaluate clinical significance through segregation studies and those deemed clinically significant then progress to predictive testing in family members. As most variants are novel and require unique test design, this poses a significant problem for diagnostic laboratories. Method: A cohort of previously designed amplicons was assessed for GC content of the primers and the intervening sequence, and their relative performance evaluated in a single PCR system. Data from this initial analysis was used to develop an amplicon design system, which stratified all unique amplicons into one of three PCR systems based on low, moderate or high GC content. Results: 2654 amplicons have been designed to date; a priori stratification based on GC content has resulted in $2572(97 \%)$ working successfully on first attempt and the remaining $82(3 \%)$ working successfully with redesign. Prediction of the most suitable PCR method is $100 \%$ successful. Conclusion: Using our approach of stratifying amplicons into PCR systems based on GC content we have been able to streamline 2,654 unique designs into only 3 methods within our laboratory, which we are able to predict with precision. This has enabled high throughput, efficient, cost effective testing of over 4500 individuals from more than 1800 families and has resulted in a significant improvement to turnaround times (median 10 days) despite an increased number of test requests.

\section{IDENTIFYING THE GENETIC CAUSE OF FAMILIAL PARKINSON'S DISEASE IN A HIGHLY TRIAGED COHORT OF PATIENTS}

\section{Hall $R^{1}$, Wilcox $\mathrm{R}^{2}$, Grist $\mathrm{S}^{\prime}$}

${ }^{1}$ SA Pathology, Bedford Park, Australia

2 Flinders Medical Centre, Bedford Park, Australia

Background: Parkinson's disease is the second most common neurodegenerative disorder, affecting 1 in 350 Australians, with more than 30 new diagnoses each day and 6.3 million people affected worldwide. Clinically, Parkinson's disease has significant overlap with many other movement disorders including dystonia, frontotemporal dementia, motor neuron disease and paroxysmal dyskinesia, making diagnosis challenging. Identification of a genetic cause for heritable Parkinson's disease may provide unaffected family members with the opportunity for presymptomatic or prenatal testing, and the possibility of individualized therapy. Methods: A cohort of 20 patients with familial Parkinson's disease was selected by a movement disorder expert. A panel of 70 genes causatively implicated in Parkinson's disease, dystonia, frontotemporal dementia, motor neuron disease or paroxysmal dyskinesia was interrogated using Next Generation Sequencing. Single nucleotide changes, small insertions and deletions and copy number abnormalities were assessed and classified using ACMG guidelines. Results: Potential disease causing variants were identified in $18 / 20$ of the familial Parkinson's disease patients. The majority of variants identified were novel, with insufficient data to definitively classify the variants. Conclusion: A targeted movement disorder panel applied to a well triaged cohort of patients was highly effectively at identifying potential disease causing variants. However, without a definitive mechanism of pathogenicity or strong supporting functional data, the clinical benefit to family members remains limited. As our capacity to identify genetic variants increases, so to must our proficiency in interpreting the clinical implications of these changes.

\section{EXON SKIPPING IN BRAT1 IN A PATIENT WITH INTELLECTUAL DISABILITY, EPILEPSY AND DYSMORPHIC FEATURES}

Heron $\mathrm{S}^{1}$, MacLennan $\mathrm{S}^{2,3}$, Thompson $\mathrm{E}^{3,4}$, Smith $\mathrm{N}^{2,3}$

${ }^{I}$ Neurogenetics Group, Adelaide Medical School, University of Adelaide, Adelaide, Australia

${ }^{2}$ Department of Neurology and Clinical Neurophysiology, Women's and Children's Hospital, Adelaide, Australia

${ }^{3}$ Department of Paediatrics and Reproductive Health, Adelaide Medical School, University of Adelaide, Adelaide, Australia

${ }^{4}$ SA Clinical Genetics Service, Directorate of Genetics and Molecular Pathology, SA Pathology, Adelaide, Australia

Background: Autosomal recessive mutations in BRAT1, coding for BRCA1-associated ATM activator 1, cause BRAT1-associated neurodegenerative disease, which manifests as epileptic encephalopathy with progressive microcephaly, intellectual disability, and premature death in infancy. Phenotypic variability is increasingly recognised, with patients with a less severe disease course now described. While the specific functional consequences of pathogenic mutations are implicated in this phenotypic variability, genotypephenotype correlation remains largely untested. Results: We report compound heterozygous mutations in BRAT1 identified by clinical whole exome sequencing in a patient with severe intellectual disability, pharmacoresistant epilepsy, microcephaly, spastic quadriparesis, dystonia and dysmorphic features. The mutations consisted of a novel splice site mutation, c. $1597+1 \mathrm{G}>\mathrm{A}$, and a previously reported missense mutation, c.1925C $>$ A; p.Ala642Glu. Reversetranscription PCR and Sanger sequencing of skin fibroblast mRNA showed that the novel splice site mutation leads to skipping of exon 12 in the mutant BRAT1 transcript. This leads to the deletion of 33 amino acids from the BRAT1 protein, including one of two HEATrepeat domains located in the $\mathrm{C}$-terminal region. In silico predictions indicate that the missense mutation is likely to have a relatively mild effect on protein function, which may contribute to the less severe phenotype seen in this patient. Conclusion: This case expands the spectrum of attenuated BRAT1-associated neurodegenerative disease and illustrates the value of in vitro analysis in understanding the functional effects of splice-site mutations. Protein expression and functional analyses are proceeding in a cohort of attenuated patients, with a view to further understanding the basis of phenotypic variability in BRAT1-related disease.

\section{A SERENDIPITOUS JOURNEY TO A PROMOTER OTC VARIANT}

Hertzog $A^{1,2}$, Fisk $K^{\prime}$, St Heaps $L^{\prime}$, de Jong $G^{3}$, Bennetts $B^{1,4}$

${ }^{1}$ Sydney Genome Diagnostics, Western Sydney Genetics Program, The Children's Hospital at Westmead, Sydney, Australia

${ }^{2}$ NSW Biochemical Genetics Service, Western Sydney Genetics Program, The Children's Hospital at Westmead, Sydney, Australia

${ }_{3}^{3}$ Metabolic Diseases Unit, Royal Melbourne Hospital, Parkville, Australia

${ }^{4}$ Detabolic Diseases Unit, Royal Melbourne Hospital, Parkville, Australia
${ }^{5}$ Detic Medicine and Paediatrics and Child Health, The University of Sydney, Sydney, Australia

Introduction: OTC deficiency (OTCD) is an X-linked disorder characterized by the partial, or complete, lack of the OTC enzyme in the urea cycle, resulting in hyperammonemia. Approximately $80 \%$ of causative variants are identified in the OTC gene, with $20 \%$ still unknown. Method: A 23-year-old male with 'presumed OTC' was referred to Molecular Genetics, CHW for variant analysis. Initial Sanger sequencing yielded no pathogenic variant, with subsequent MLPA testing leading to the detection of an apparent mosaic deletion of exon 1. With no apparent variant within the probe binding site, high resolution chromosome $\mathrm{X}$ microarray was employed for 
further elucidation of the apparent CNV. This found no CNV involving the OTC gene or flanking sequences within chromosome

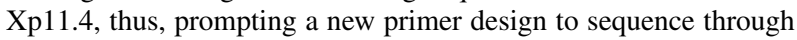
the presumably small deletion breakpoint. Results: No deletion was detected, however, a hemizygous likely pathogenic promoter variant, c. $-106 \mathrm{C}>\mathrm{A}$, was identified. Being upstream of the MLPA exon 1 probe binding site, it is predicted to be the cause of the reduced $(25 \%)$ signal of this probe. Recent reports have identified this variant in 4 unrelated males, and functional analysis strongly suggests this could be the cause of the OTCD in these patients. Conclusion: This case highlights the challenges faced with obtaining a molecular diagnosis, the limitations of various techniques, and the importance of a multidisciplinary approach when trying to confirm a clinical diagnosis. This case raises the possible need for the routine screening of promoter variants within the OTC gene.

\section{COMPARISON OF VARIANTS DETECTED USING ILLUMINA TRUSIGHT ONE AND AGILENT SURESELECT CLINICAL RESEARCH EXOME V2}

Ho $\mathrm{G}^{1,2}$, Bennetts $\mathrm{B}^{1,2}$

${ }^{I}$ Sydney Genome Diagnostics, Western Sydney Genetics Program, Children's Hospital Westmead, Westmead, Australia

${ }^{2}$ Discipline of Child \& Adolescent Health; Discipline of Genetic Medicine, University of Sydney, Sydney, Australia

Background: Massively parallel sequencing (MPS) has become an important tool in molecular diagnosis. Since 2014, the Molecular Genetics laboratory has utilized the Illumina TruSight One panel $(4,813$ genes $)$ as the capture method for the basis of testing for a range of Mendelian disorders; for example, epileptic encephalopathies, aortopathies, renal, immunological and metabolic disorders. However, there is increasing clinical need for whole exome capture as new candidate genes are identified but are not targeted by the TruSight One panel. Aim: To compare variants detected by Illumina TruSight One against those detected by whole exome sequencing. Method: Eight samples referred for diagnostic testing were tested using two capture methods: Illumina TruSight One and Agilent SureSelect Clinical Research Exome v2 (CREv2), latter performed at Genome.One (Sydney, Australia). Alignment and variant calling were performed using NextGene (SoftGenetics). $R e$ sults: Within the regions targeted by both captures, an average of 7111 variants was called using CREv2, compared to 7287 variants using TruSight one, with 6753 variants concordant between the two assays. Of the discordant calls, approximately $20 \%$ were due to insufficient coverage in one of the assays. Another $3 \%$ were discordant in the zygosity call between assays. TruSight One capture also resulted in a higher number of false positives. Conclusion: The use of the Agilent CREv2 will allow for recently discovered genes to be added to the current testing catalogue, and also provide for the ability to reinterrogate the data as other candidate genes are identified.

\section{POLYMORPHISMS IN GENES LINKED WITH FOLATE METABOLIC PATHWAY IN CZECH CHILDREN WITH DENTAL CARIES}

Borilova Linhartova $P^{1,2}$, Mrazkova J', Novak $D^{1}$, Hulova $D^{4}$, Kukla $L^{3}$, Kukletova $M^{\prime}$, Izakovicova Holla L ${ }^{1,2,3}$

${ }^{\prime}$ Institution Shared with St. Anne's Faculty Hospital, Faculty of Medicine, Masaryk University, Brno, Czech Republic, Brno, Czech Republic

${ }^{2}$ Department of Pathophysiology, Faculty of Medicine, Masaryk University, Brno, Czech Republic, Brno, Czech Republic

${ }^{3}$ Research Centre for Toxic Compounds in the Environment (RECETOX), Chemistry

Section, Faculty of Science, Brno, Czech Republic

${ }^{4}$ Faculty Hospital Brno, Czech Republic, Brno, Czech Republic

Background: Dental caries is a multifactorial, infectious disease where genetic predisposition can play an important role. Its susceptibility may be modified by functional polymorphisms in genes linked with the folate metabolic pathway, such as methionine synthase
(MTR), methionine synthase reductase (MTRR), and methylenetetrahydrofolate reductase (MTHFR). The aim of this study was to analyze four single nucleotide polymorphisms (SNPs) in the genes for these enzymes in the group of caries free children vs. subjects affected by dental caries in the Czech population. Methods: This casecontrol study included 1,017 subjects: 145 caries-free children (with decayed/missing/filled teeth DMFT $=0$ ) and 466 subjects with dental caries (DMFT $\geq 1$ ) aged 13-15 years from the European Longitudinal Study of Pregnancy and Childhood (ELSPAC) and 282 children aged 2-6 years with early childhood caries (ECC, dmft $\geq 1)$ together with 124 caries-free children $(\mathrm{dmft}=0)$. MTHFR C677T (rs 1801133), MTHFR A1298C (rs1801131), MTR A2756G (rs1805087) and MTRR A66G (rs1801394) SNPs were determined by qPCR. Results: Although no significant differences in the allele or genotype frequencies of all four SNPs between the cariesfree children and those affected by ECC were observed $(p>.05)$, MTHFR 1298 AA vs. AC+CC and MTHFR 677 TT vs. CT + CC genotypes were found as protective factor for dental caries $(p>$ .05 and $p<.01$, respectively) in children with permanent dentition. There were no significant differences in the allele or genotype frequencies in both MTR and MTRR SNPs between these patients and healthy controls $(p>.05)$. Conclusions: MTHFR polymorphisms may be associated with caries protection in permanent but not primary dentition.

\section{CRANIO-FRONTO-NASAL SYNDROME IN A PATIENT WITH A PERICENTRIC INVERSION OF THE X CHROMOSOME}

Kakadia $\mathrm{P}^{\mathrm{I}}$, Fritz $\mathrm{B}^{2}$, Bohlander $\mathrm{S}^{\mathrm{I}}$

${ }^{I}$ Leukaemia \& Blood Cancer Research Unit, Department of Molecular Pathology, Faculty of Medical and Health Sciences, University of Auckland, Auckland, New Zealand

2 Institute of Human Genetics, Philipps University Marburg, Marburg, Germany

Craniofrontonasal syndrome (CFNS, MIM 304110) is an X-linked developmental disorder caused by mutations in the ephrin B1 (EFBN1) gene located on Xq13.1. Counterintuitively, heterozygous females have a severe phenotype, whereas hemizygous male EFNB1 mutation carriers only exhibit hypertelorism. We report a female CFNS patient who was diagnosed with the typical features of CFNS as a newborn. Her karyotype revealed a de novo pericentric inversion of one X chromosome (46,X,inv(X)(p22.2q12)). Her facial malformations were corrected in multiple surgeries. At the age of 30 , the patient presented to our genetic counseling unit inquiring about preimplantation genetic diagnostics. Molecular testing for EFNB1 mutations and a SNP-array test for genomic imbalances showed neither mutations in the coding regions of EFNB1 nor genomic imbalances. In order to identify the inversion breakpoints, we performed low pass whole genome sequencing generating 200 million $150 \mathrm{bp}$ reads ( $150 \mathrm{bp}$ paired end, 30 gigabases, $5 \mathrm{x}$ haploid coverage). One $150 \mathrm{bp}$ read spanned the putative short arm inversion breakpoint. The short arm and the inferred long arm inversion breakpointswere confirmed with specific breakpoint spanning PCRs and Sanger sequencing of the PCR products (ChrX: g.14593188_68159452inv; hg19). The long arm inversion breakpoint is about $100 \mathrm{kbp}$ upstream of the 5' end of the EFNB1 gene, potentially separating an enhancer region from EFBN1. The short arm breakpoint disrupts the GLRA2 gene most likely leading to a complete loss of function of GLRA2. To our knowledge, this is the first example of CFNS potentially caused by a long-range genomic rearrangement. 


\section{Y CHROMOSOME STRUCTURAL ABNORMALITIES AND RELATED PHENOTYPES - MONASH PATHOLOGY RETROSPECT REVIEW}

Krishnaswamy R', Kulkarni A', Gugasyan L'

${ }^{1}$ Monash Pathology, Monash Medical Centre, Clayton, Australia

Structural abnormalities involving the $\mathrm{Y}$ chromosome include deletions, inversions, isochromosomes, ring chromosomes and translocations. Phenotypes associated with these rearrangements can vary greatly from normal male with/without fertility issues to ambiguous genitalia to females resembling testicular feminization or displaying features of Turner syndrome. In addition to the nature of the rearrangement, the clinical consequences will be effected by the presence of other cell lines such as 45,X, and the tissue distribution and proportions of abnormal cell lines when observed in mosaic form. A combination of conventional chromosomal and molecular testing may be required to elucidate the morphology and extent of loss/gain of an abnormal Y chromosome. Certain anomalies such as satellited Yqh and pericentric inversions tend to be familial variants. A retrospect review of 76 cases with structurally abnormal Y chromosome identified pre- and postnatally over the last two decades at Monash Pathology was undertaken. Proportions of the more common abnormalities were found to correlate with other published data.

\section{REVIEW OF AORTOPATHY GENE TESTING BY MASSIVELY PARALLEL SEQUENCING (MPS)}

\footnotetext{
Lai $\mathrm{T}^{1}$, Ho $\mathrm{G}^{1,2}$, Holman $\mathrm{K}^{1}$, Bennetts $\mathrm{B}^{1,2}$, Adès $\mathrm{L}^{1,2}$

${ }^{1}$ Sydney Genome Diagnostics, Western Sydney Genetics Program, Children's Hospital at Westmead, Westmead, Australia

${ }^{2}$ Discipline of Child \& Adolescent Health; Discipline of Genetic Medicine, University of Sydney, Sydney, Australia
}

Background: Aortopathy genes are associated with aortic dilatation and dissection and consist of a range of disorders including Marfan syndrome, Loeys-Dietz syndromes, familial thoracic aortic aneurysms and dissections and other related disorders. These disorders share overlapping clinical features and phenotypes can be variable across the same variant and also different variants within the one gene. Previously, genetic testing involved Sanger sequencing of genes performed in a sequential manner; posing a challenge as to which gene to test first. Through massively parallel sequencing (MPS) a large panel of genes can be sequenced simultaneously. Aim: To review aortopathy patients tested diagnostically by MPS to investigate the spectrum of genes and variants associated with this phenotype. Methods: MPS was performed using the Illumina TruSight One panel on an Illumina HiSeq 2500 or NextSeq 550. Alignment and variant calling were performed using NextGene. Results: Since 2014 over 600 patients have been tested for the aortopathy panel ( 20 genes) and over 40 patients for the Ehlers Danlos panel (15 genes). Variants have been identified in genes such as FBN1, TGFBR1 \& TGFBR2 and SMAD3. Discussion/Conclusion: MPS has been an effective diagnostic tool to identify causative mutations. However, the number of genes associated with aortic dilatation and dissections continues to expand and, for this reason, testing for aortopathy and EDS panels will be performed using a whole exome capture. This will allow the data to be reinterrogated for other genes or panels as a patient's phenotype may evolve over time and new causative genes are discovered.

\section{INCREASING THE EFFICACY AND ACCURACY OF THE CRISPR-CAS9 SYSTEM USING ARTIFICIAL INTELLIGENCE}

Ludusan $\mathrm{M}^{\prime}$

${ }^{I}$ Queensland Health, Brisbane, Australia

The CRISPR-CAS9 gene editing system is a revolutionary new molecular technology that has the potential to cure all genetic diseases. Laboratories around the world are developing ingenious ways to apply this new molecular tool. So far researchers have used CRISPR to cure muscular dystrophy in mice, to alter immune cells in human subjects with lung cancer, and in the ambitious and controversial feat of rendering entire mosquito populations immune to malaria using gene drives. The aim of this talk is to look into the serendipitous way that CRISPR was discovered, describe molecularly how CRISPR and Gene Drives work and look into the current and future application of this technology when used in conjunction with recent advances in the field of artificial intelligence. The main challenge for researchers using CRISPR is trying to decide what guide RNA to choose in any given experiment. For each gene knockout target of CRISPR there are hundreds of potential 20 nucleotide RNA guides. My talk will focus on one particular computational tool designed by a collaboration of scientists and biologists from MIT, Berkeley and the Broad institute called Elevation. This AI system increases the efficiency and accuracy of CRISPR by analysing offtarget effects. Elevation provides researchers with probabilities that something bad will happen for every single gene region targeted by the RNA guides in order to avoid unintentional and severe consequences such as knocking out off-target genes. Additionally, I will compare Elevation with other AI systems designed to increase the accuracy of gene editing systems.

\section{WGS IN ANTERIOR SEGMENT DYSGENESIS AND CATARACTS REVEALS STRUCTURAL VARIANTS IN PREVIOUSLY UNSOLVED CASES}

Ma $A^{1,2}$, Minoche $A^{3}$, Grigg J,4, Flaherty $M^{5}, \mathrm{Ho} \mathrm{G}^{2,6}$, Amor $D^{7}$, Cheng $A^{1,2}$, Bennetts $B^{2,6}$, Cowley $M^{3,8}$, Dinger $M^{3,8,9}$, Jamieson $R^{1,2,4}$

${ }^{I}$ Eye Genetics Research Unit, Sydney Children's Hospital Network, Children's Medical Research Institute, University of Sydney, Sydney, Australia

${ }^{2}$ Discipline of Genetic Medicine, CHW Clinical School, Sydney Medical School, University of Sydney, Sydney, Australia

${ }^{3}$ Kinghorn Centre for Clinical Genomics, Garvan Institute for Medical Research, Sydney, Australia

${ }^{4}$ Save Sight Institute, University of Sydney, Sydney, Australia

${ }^{5}$ Department of Ophthalmology, The Children's Hospital at Westmead, Sydney, Australia

${ }^{6}$ Sydney Genome Diagnostics, Sydney Children's Hospital Network, Westmead, Sydney, Australia

${ }^{7}$ Murdoch Children's Research Institute and University of Melbourne Department of Paediatrics, Royal Children's Hospital, Melbourne, Australia

${ }^{8}$ St Vincent's Clinical School, UNSW Sydney, Sydney, Australia

${ }^{9}$ Genome.One, Sydney, Australia

Introduction: Disorders of the ocular anterior segment exhibit marked clinical and genetic heterogeneity. We have previously obtained a genetic diagnosis in a high proportion of patients utilising an NGS-based panel or whole exome sequencing (WES) approach and CGH microarray, finding answers in over 70\% (33/46) of congenital cataract patients, and almost 40\% (14/39) of patients with anterior segment abnormalities. Methods: In this study we applied whole genome sequencing (WGS) to assist with 38 cases (13 congenital cataracts, 25 anterior segment abnormalities) unsolved after CGH microarray, and panel or WES. WGS utilized the Illumina HiSeq X Ten and a bioinformatics structural variant analysis pipeline, ClinSV, which allows confident detection of rare structural and copy number variants, using evidence from split-reads, discordant pairs and depth-of-coverage. Results: A causative variant was found in 3/38 patients, who harboured deletions too small to be seen on conventional microarray or capture-based sequencing. A novel 
$3.2 \mathrm{~kb}$ deletion in the MIP gene was found in a patient with congenital cataracts and microphthalmia. A $1 \mathrm{~kb}$ deletion in PAX6 was found in a family with autosomal dominant anterior segment dysgenesis. A $4 \mathrm{~kb}$ deletion in the X-linked syndromal gene NHS was found in a male with congenital cataracts and coloboma. Discussion: This study demonstrates an increased yield of genetic diagnoses in a cohort of patients with complex ocular phenotypes. While the yield of CGH microarray combined with panel/exome based testing is significant, WGS analysis revealed additional diagnoses of structural variants, providing new diagnostic and management information.

\section{WITHDRAWN}

\section{WITHDRAWN}

\section{NOVEL STRATEGIES FOR IDENTIFYING FAMILIAL MOTOR NEURON DISEASE GENES USING NEXT GENERATION SEQUENCING AND LINKAGE}

McCann $E^{\prime}$, Fifita J', Zhang $K^{\prime}$, Freckleton $S^{\prime}$, Williams $K^{\prime}$, Lyu $R^{2}$, Twine $N^{\prime, 2}$, Bauer D', Rowe D', Blair I'

${ }^{1}$ Centre for MND Research, Macquarie University, Department of Biomedical Sciences, Macquarie University, Sydney, Australia

${ }^{2}$ Health \& Biosecurity Flagship, Commonwealth Scientific and Industrial Research Organization, Sydney, Australia

Motor neuron disease (MND) is a fatal, genetically heterogeneous neurodegenerative disease. Patients gradually lose control of their muscles so that they can no longer talk, walk, eat or breathe. Approximately $10 \%$ of cases are hereditary. Gene mutations are the only proven cause, yet one-third of MND families carry an unidentified disease-causing mutation. These families are often small and exhibit incomplete disease penetrance, inhibiting traditional gene mapping. We developed a pipeline utilizing next generation sequencing (NGS) and linkage analysis to identify MND mutations within these families. We applied this pipeline to one such family, with DNA available from two patients and an obligate carrier, all of whom underwent whole-exome (WES) and whole-genome sequencing (WGS). Shared variant analysis and extensive control filtering resulted in 15 (WES) and 12 (WGS) coding candidate variants; however, Sanger sequencing revealed all to be false positives. Linkage analysis in an extended pedigree identified 40 and 431 genomic regions respectively exhibiting positive or non-exclusion disease linkage scores. Shared variant analysis was expanded to consider regulatory elements in these regions. One (WES) and 12 (WGS) novel candidates from positive linkage regions were also false positives, while nine further candidates from non-exclusion linkage regions remain to be validated. Copy number variant (CNV) analysis also identified candidate $\mathrm{CNVs}$ among the three informative family members. Preliminary control filtering revealed one ncRNA-exonic variant as an interesting pathogenic candidate. While gene discovery in MND is hindered by reduced disease penetrance, our comprehensive strategy offers hope for identifying the remaining familial MND genes.

\section{A COMPARATIVE ANALYSIS OF PREIMPLANTATION GENETIC TESTING TECHNIQUES PAST AND PRESENT AT SULLIVAN NICOLAIDES PATHOLOGY}

McReight $\mathrm{E}^{1,2}$, Brookwell $\mathrm{R}^{1}$, Barton $\mathrm{N}^{1,2}$, Anderson J'

${ }^{I}$ Sullivan Nicolaides Pathology, Australia

2 Illumina, San Diego, USA

Preimplantation Genetic Testing (PGT) is a rapidly growing area in the Cytogenetic Department of Sullivan Nicolaides Pathology (SNP). Until the mid-2000s, FISH probes were used to identify aneuploidy of chromosomes $13,16,18,21,22, \mathrm{X}$ and $\mathrm{Y}$, with relative success. Post FISH, PGT technology advanced to using the Illumina 24 Sure comparative genomic hybridization (CGH) microarray plat- forms. These techniques allowed screening of whole genome amplified (WGA) DNA from single embryo cells and multiple trophectodermal cells to detect whole chromosome aneuploidy and large copy number variations variants $(>10 \mathrm{Mb})$, with high levels of reproducibility and sensitivity. These microarray systems have been discontinued and replaced with the VeriSeq Preimplantation Genetic Screening (PGS) assay. Veriseq uses low pass whole genome massively parallel sequencing to detect whole chromosome aneuploidy in WGA-amplifed products, and has recently been verified by our laboratory. During this verification process a comparative analysis was completed between the previous CGH microarray and the Next generation sequencing (NGS) PGT techniques, providing assurance that the transition would be easily facilitated and beneficial. This analysis aimed to determine how VeriSeq NGS compares to 24Sure CGH array as a PGT technique. Information on the two PGT techniques, including laboratory and analysis procedure, costing and previous analyses, was gathered from multiple sources to compare the two PGT techniques. It was concluded that the VeriSeq NGS PGT procedure is a more cost effective and reliable PGT technique for SNP and the transition between the two procedures is easily facilitated due to common laboratory and analysis techniques.

\section{SILENT CARRIER OF BETA ZERO THALASSEMIA RESULTING FROM CO-INHERITANCE WITH DELTA ZERO THALASSEMIA}

Nicholls $C^{\prime}$, Simsek $A^{\prime}$, Thompson $E^{2}$

${ }^{1}$ SA Pathology, Adelaide, Australia

${ }^{2}$ The Women's and Children's Hospital, Adelaide, Australia

Background: Both beta and delta-beta thalassemia trait are characterized by microcytic, hypochromic indices, with delta-beta thalassemia having a normal $\mathrm{HbA} 2$, but significant elevation of $\mathrm{HbF}$ $(>5 \%)$, and beta thalassemia having raised $\mathrm{HbA} 2(3-6 \%)$. Here we report a case of beta zero and delta zero thalassemia variants co-inherited in trans resulting in a silent carrier of beta zero thalassemia with normal $\mathrm{HbA} 2$ and a only a slight elevation of $\mathrm{HbF}$. Methods: Common HBA (hemoglobin alpha) deletions were detected by gap PCR. Variants in the HBA, HBB and HBD genes were detected by sequence analysis, with deletions detected using MLPA. Results: A 2-year old boy with a microcytic, hypochromic anemia and normal HbA2 was referred for investigation of alpha thalassemia. Gap PCR sequence analysis and MLPA of his HBA genes were all normal. Sequence analysis of the HBB gene detected heterozygosity for beta zero thalassemia. No HBA or HBB variants were detected in his mother, however his father was found to be heterozygous for the same beta zero variant, but with raised $\mathrm{HbA} 2$. Beta globin gene MLPA in the mother and son detected heterozygosity for a large deletion in the HBD gene in both (NG_000007.3: g.[(?_59683)_(63149_?)del];[=]). Conclusions: Co-inheritance of the paternal beta zero variant and the maternal delta- zero thalassemia variant in trans has meant that this child is a silent carrier of beta zero thalassemia with a normal $\mathrm{HbA} 2$. This combination of gene variants has consequences for genetic counseling in first degree relatives.

\section{ATYPICAL HBH DISEASE IN A SUDANESE CHILD: A NOVEL ALPHA ZERO DELETION}

\section{Simsek $A^{\prime}$, Teo $J^{2}$, Nicholls $C$}

${ }^{I}$ Genetics and Molecular Pathology, SA Pathology, Adelaide, Australia

${ }^{2}$ The Children's Hospital at Westmead, Westmead, Australia

Background: Normal individuals have four hemoglobin alpha genes, two on each chromosome (HBA2 and HBA1). Carriers of alpha thalassemia have one gene inactivated (alpha plus thalassemia) or two genes inactivated in cis (alpha zero thalassemia). HbH Disease patients have three genes inactivated and patients with Barts Hydrops 
Fetalis have all four genes inactivated. In Africa single gene deletions are common (5\%), but alpha zero, rare. We report a case of $\mathrm{HbH}$ disease in a Sudanese child resulting from co-inheritance of a paternal single gene deletion with a novel maternal two gene deletion. In addition, mother and child both carry an HBD (hemoglobin delta) gene variant which lowers the percentage of hemoglobin A2 (HbA2). Methods: A Sudanese child with indices consistent with $\mathrm{HbH}$ disease and his mother were screened by gap PCR for seven common HBA deletions. Uncommon deletions were screened by MLPA. Sequence analysis characterized the deletion breakpoints. Results: Gap PCR detected a paternally inherited single gene deletion in the child. A novel maternal two gene deletion was detected by MLPA and characterized as NG_000006.1:g.32380-41312. MLPA in the child generated a complex 3 gene deletion pattern consistent with compound heterozygosity for the maternal two gene deletion and the paternal single gene deletion. Sequence analysis detected the HBD missense variant, HBD:c.68C $>$ A (p.Ala23Glu, Hb A2 Flatbush) in the mother and child, explaining the low $\mathrm{HbA} 2$ levels in the mother $(1.1 \%)$. Conclusion: This is the first report of $\mathrm{HbH}(3$ gene deletion) in a Sudanese family with a co-inherited HBD gene variant.

\section{FUNCTIONAL GENOMIC, RETINAL ORGANOID AND MOUSE MODEL STUDIES IN CHARACTERIZATION OF A NOVEL RETINAL CILIOPATHY}

\begin{abstract}
Sabri $A^{\prime}$, Loi $T^{\prime}$, Cheng $A^{\prime}$, Van Den Bergh $M^{\prime}$, Karam $F^{\prime}$, Grigg J', , Jamieson $R^{1,3}$ ${ }^{I}$ Eye Genetics Research Unit, Children's Medical Research Institute, The Children's Hospital at Westmead, Save Sight Institute, University of Sydney, Australia

${ }^{2}$ Discipline of Ophthalmology, University of Sydney, Sydney, Australia

${ }^{3}$ Disciplines of Genetic Medicine, and Child and Adolescent Health, University of Sydney, Sydney, Australia
\end{abstract}

Background: Inherited retinal dystrophies (IRDs) may be syndromic or non-syndromic, and many are caused by variants in genes affecting primary cilia structure or function of the photoreceptors, which are specialized sensory cilia. Through genomic analysis in a newly identified syndromic form of retinal dystrophy, we have identified a novel candidate kinase disease gene with a predicted role in centrosomal biology and ciliary function. Methods: Cell-based, human induced pluripotent stem cell (hiPSC)-derived retinal pigment epithelium (RPE), retinal organoid and animal model approaches were undertaken to understand the function of the novel disease gene. A CRISPR/Cas9 generated mouse model was investigated using electroretinography (ERG) and immunohistochemistry. Results: Analyses in mouse retina showed expression of the protein in the connecting cilium region of the photoreceptors with a possible role in ciliary trafficking. Immunolabelling of the hiPSC-derived retinal tissues confirmed the expression of the protein in the basal body of the primary cilium. Embryonic fibroblasts established from mutant mice showed decreased proportion of ciliated cells. ERG studies revealed significant decrease in scotopic and photopic responses in mice with the orthologous mouse mutation, and histology sections showed thinning of the retinal layers. Further immunohistochemistry studies confirmed aberration of connecting cilium proteins, Ift 88 and centrin, in the mutant mouse photoreceptors. Conclusion: Through functional genomic studies, we have shown that this newly identified candidate disease gene is critical for normal ciliary function in the photoreceptors. This study highlights the value of combined hiPSC-derived retinal and mouse model studies for detailed disease gene characterization in the IRDs.

\section{STRENGTHS AND CAVEATS OF PARALLEL SEQUENCING APPROACHES IN NEMALINE MYOPATHY}

Sandaradura $S^{1,2,3}$, Joshi $H^{1}$, Davis $M^{4}$, Bryen $S^{1}$, Kaur $S^{5}$, Waddell $L^{1,2}$,

O'Grady $G^{1,6}$, Ghaoui $R^{1,7}$, Cairns $A^{8}$, Ryan $M^{9,10}$, Patel $R^{6}$, Collins $F^{2,11}$, Giunta $C^{12}$, Lek $M^{13,14,15}$, Laing $N^{4,16}$, Jones $K^{1,2,3}$, Cummings $B^{13,14}$, MacArthur $\mathrm{D}^{13,14}$, Clarke $\mathrm{N}^{1,2}$, North $\mathrm{K}^{1,5,10}$, Cooper $\mathrm{S}^{1,2}$

${ }^{1}$ Institute for Neuroscience and Muscle Research, The Children's Hospital at Westmead, Sydney, Australia

${ }^{2}$ Discipline of Child and Adolescent Health, University of Sydney, Sydney, Australia

${ }^{3}$ Department of Clinical Genetics, Children's Hospital at Westmead, Sydney, Australia

${ }^{4}$ Department of Diagnostic Genomics, PathWest Laboratory Medicine, QEII Medical

Centre, Perth, Australia

${ }_{5}^{5}$ Murdoch Children's Research Institute, Melbourne, Australia

${ }^{6}$ Paediatric Neuroservices, Starship Children's Health, Auckland, New Zealand

${ }^{7}$ Royal Adelaide Hospital, Department of Neurology, Adelaide, Australia

${ }^{8}$ Lady Cilento Children's Hospital, Brisbane, Australia

9 Depatment of Neurology, Royal Children's Hospital, Melbourne, Australia

${ }^{10}$ Department of Paediatrics, Faculty of Medicine, University of Melbourne, Melbourne, Australia

${ }^{11}$ Department of Clinical Genetics, Royal Prince Alfred Hospital, Sydney, Australia

${ }_{12}$ Division of Metabolism, University Children's Hospital and Children's Research

Centre, Zurich, Switerland

${ }^{13}$ Analytic and Translational Genetics Unit, Massachusetts General Hospital, Boston USA

${ }^{14}$ Broad Institute of Harvard and Massachusetts Institute of Technology, Cambridge,

USA

${ }_{15}$ Department of Medical Genetics. Yale School of Medicine, New Haven, USA

${ }^{16}$ Centre for Medical Research University of Western Australia; Harry Perkins Institute of Medical Research, Perth, Australia

Background: Nemaline myopathy (NM) is a form of congenital myopathy defined histopathologically by the feature of nemaline bodies on skeletal muscle biopsy. The traditional approach to diagnosis has been by skeletal muscle biopsy and candidate gene sequencing. Aim: To determine the diagnostic utility of different massively parallel sequencing (MPS) approaches in NM. Methods: A cohort of 21 individuals with $\mathrm{NM}$ were investigated using a range a different MPS techniques (neuromuscular gene panel, whole exome sequencing (WES), whole genome sequencing (WGS), RNA-Sequencing (RNA-Seq). Results: A genetic diagnosis was obtained in 20/21 NM families investigated using MPS approaches (95\% diagnosis). Pathogenic splicing variants were identified for $6 / 20$ families; extended splice site or intronic variants relied heavily upon mRNA studies from muscle biopsies to support pathogenicity. This study redefines the relative incidence of NM genotypes in our Australian cohort of probands from 52 families with a histopathological diagnosis of NM and a molecular genetic diagnosis. Discussion/Conclusion: this study confirms the utility of massively parallel sequencing as a first-tier investigation for patients with congenital myopathy, while emphasising the continuing importance of muscle biopsy in a subset of patients. We highlight challenges interpreting variants simultaneously identified in multiple plausible candidate genes, provide specific recommendations regarding analysis and interpretation of splicing variants, and propose a diagnostic algorithm for genetic diagnosis of congenital myopathy.

\section{HIGH DIAGNOSTIC YIELD FROM MULTI-GENE PANEL FOR EPILEPSY SUGGESTS FIRST USE PRIOR TO EXOME SEQUENCING}

Scully $L^{\prime}$, Truty $R^{\prime}$, Patil N', Aradhya $S^{\prime}$

${ }^{I}$ Invitae Corporation, San Francisco, United States

Background: Genetic testing in epilepsy is clinically useful, but the mutation spectrum in individuals with epilepsy, the sources of variants of uncertain significance, and a comparison of epilepsy panels versus whole exome sequencing require deeper investigation to provide clinicians relevant evidence to guide appropriate genetic analysis in patients. Methods: We used a multi-gene next-generation sequencing (NGS) panel with simultaneous sequence and exonic copy number variant detection to investigate subsets of 189 epilepsyrelated genes in 4097 individuals. We also compared the analytic 
and clinical sensitivity of the epilepsy panel with whole exome sequencing (WES). Results: The epilepsy panel yielded a molecular diagnosis in up to $24 \%$ of individuals. Approximately $12 \%$ of clinically significant variants were intragenic copy number variants or large indels. Most variants of uncertain significance were likely not important because they were single variants in genes with autosomal recessive inheritance or were in the presence of a positive result in another gene. A meta-analysis of recent WES studies revealed that more than half of the positive results in individuals with epilepsy involved genes already present in available panels. The quality of sequence data for epilepsy genes from three exome kits demonstrated significant variation, with an overall minimum risk of $1.6 \%$ for false negative results. Conclusions: A targeted NGS panel that includes genes associated with the major causes of hereditary epilepsy and offers both sequence and exonic copy number analysis is a useful first test because of a high diagnostic yield in children with a broad variety of epilepsy phenotypes.

\section{IQSEC2 KNOCK-OUT MICE RECAPITULATE THE INTELLECTUAL DISABILITY AND EPILEPSY PHENOTYPE OF PATIENTS WITH LOSS-OF-FUNCTION MUTATIONS}

Jasckson $\mathrm{M}^{1,2}$, Loring $\mathrm{K}^{1,2}$, Shoubridge $\mathrm{C}^{1,2}$

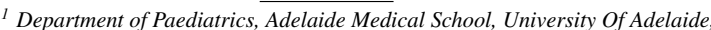
Australia

${ }^{2}$ Robinson Research Institute, University of Adelaide, Australia

The IQ motif and SEC7 domain-containing protein 2 (IQSEC2) is an X-chromosome gene mutated in both males and females leading to intellectual disability (ID) and severe early-onset seizures. The pathogenesis underpinning these mutations remains unknown. Utilising CRISPR/Cas9 targeted editing (Prof PQ Thomas, SA Genome Editing Facility, University of Adelaide) we have generated an Iqsec2 KO mouse model to investigate the molecular and cellular deficits in this gene resulting in disease outcomes; a fundamental step towards the design and implementation of potential treatment options. We confirmed the loss of Iqsec 2 mRNA expression and the lack of Iqsec 2 protein detected within the brain of founder and progeny mice. Recapitulating the human setting, both male (48\%) and female (45\%) Iqsec $2 \mathrm{KO}$ mice present with frequent and recurrent seizures. There was an increased occurrence of seizures, reabsorption and unsuccessful nurturing of live young in breeding females. Developmentally, the KO mice exhibit significantly increased hyperactivity, altered anxiety and fear responses, decreased social interactions, delayed learning capacity and decreased memory retention/novel recognition; recapitulating the psychiatric issues, autistic-like features and cognitive deficits present in patients with loss-of-function IQSEC2 mutations. Interestingly, the loss of Iqsec 2 function not only causes severe ID and seizures in KO male mice, but in agreement with the patient setting, similar severity is also noted in females despite being in a heterozygous state for this X-chromosome gene. We contend this newly generated mouse model provides a highly relevant biological tool required to interrogate IQSEC2/Iqsec2 function in the brain.

\section{TRANSCRIPTOME-WIDE RNA SEQUENCING ANALYSIS} OF IMMOBILIZATION-INDUCED MUSCLE ATROPHY

\section{Thompson $\mathrm{J}^{\prime}$, Budiono $\mathrm{B}^{\prime}$, Shike $\mathrm{K}^{\prime}$, Doering $\mathrm{T}^{\prime}$, Coffey $\mathrm{V}^{\prime}$, Ashton $\mathrm{K}^{\prime}$}

${ }^{I}$ Bond University, Varsity Lakes, Australia

Introduction: Disuse atrophy is a secondary complication that often exacerbates the etiology of injury and chronic disease. Identifying changes in mechanisms that control muscle mass is necessary to characterize atrophy and maintain a healthy functional capacity. Aim: To use RNA sequencing as a high resolution, untargeted approach to study gene expression in human skeletal muscle follow- ing short-term limb immobilization. Methods: Twenty-one healthy male participants (20-39 years) completed 4 weeks of standardized physical activity prior to a 14-day limb (left leg) immobilization period with dietary control. Skeletal muscle biopsies were collected from the $\mathrm{m}$. vastus lateralis before and after 3 days and 14 days of immobilization. Skeletal muscle RNA was isolated and analyzed using Illumina RNA sequencing. Strength testing, DEXA and MRIs were also performed pre- and post-immobilization. Results: Strength $(-16.4 \%)$, lean mass $(-3.2 \%)$ and quadricep crosssectional area $(-8.5 \%)$ were all significantly reduced after 14 days. RNA sequencing identified significant changes in mitochondrial processes and carbohydrate metabolism, which became more pronounced throughout immobilization. Furthermore, changes in chromatin remodelling processes appeared to be transient changes, as they were only observed at 3 days. Conversely, changes in protein localization, ribosome biogenesis, translation, nucleotide metabolism and oxidoreductase activity were only identified after 14 days. Conclusion: This study provides the first extensive transcriptomic sequencing of short-term disuse atrophy in human skeletal muscle. These data support mitochondrial dysfunction and reduced protein synthesis as key events during muscle disuse atrophy. Early changes in chromatin remodelling processes may be responsible for modifying transcriptomic responses to short-term disuse atrophy.

\section{INCIDENTAL/SECONDARY/ADDITIONAL FINDINGS? A SURVEY OF OPINIONS AND PRACTICES ACROSS AUSTRALIAN GENETIC TESTING LABORATORIES}

On behalf of Australian Genomics Program 2, Tudini E' , Spurdle A'

${ }^{I}$ QIMR Berghofer Medical Research Institute, Brisbane, Australia

As large panels, whole exome and whole genome sequencing increases, so arises the potential to uncover clinically relevant genetic variants unrelated to the genetic test request - variously termed 'additional', 'incidental', or 'secondary' findings. The American College of Human Genetics (ACMG) currently recommends the return of likely pathogenic/pathogenic variants in 59 medically actionable genes, irrespective of the indication for genetic testing. We conducted a survey of Australian NATA-accredited genetic testing laboratories to evaluate practices around annotation, storage, curation and reporting of secondary findings. The survey was distributed to laboratories by email, and/or via attendees of the Australian Genomics Variant Curation and Sharing workshop held at HGSA 2017. Responses were received for 13/16 (81\%) laboratories performing large panels, whole exome or whole genome sequencing. General observations to date include: (1) There is no Australian consensus on a term to describe these findings, with incidental (38\%) or secondary (38\%) findings used equally for reporting. (2) 9/13 (69\%) laboratories report secondary findings subject to patient consent; only two have adopted the ACMG-59 gene list. (3) Most laboratories (62\%) are informed of patient consent around notification of these findings at the time of test request. (4) When consent has not been sought for return of secondary findings, $23 \%$ of laboratories do not report, while $54 \%$ discuss the result with a clinician or multidisciplinary team to inform decision/s regarding reporting. (5) Findings are generally stored in laboratory databases $(85 \%)$, even if not reported. These results indicate the need to develop an Australian consensus on protocols around report of secondary findings. 


\section{WITHDRAWN}

\section{HETEROZYGOUS LOSS OF FUNCTION VARIANTS IN WDFY3 ARE ASSOCIATED WITH MACROCEPHALY, INTELLECTUAL DISABILITY AND AUTISM}

Wallis $M^{1,5}$, Le Duc $D^{2}$, Hiatt $S^{4}$, Büttner $B^{2}$, Bend $R^{3}$, Carss $K$, Cooper $G^{3}$, DeBrosse $S$, Doering $J^{7}$, Everman $D^{4}$, Jeshira Reynoso Santos $F^{9}$, Kotzaeridou $U^{7}$, Morel $D^{10}$, Payne $K^{11}$, Raymond L, Sanchis-Juan A, Smith $B^{4}$, Strober J'2, Tekin $M^{10}$, Wallis $K$, Walsh $L^{11}$, Ziegler $A^{7}$, Lemke $J^{2}$, Sticht $H^{13}$, Brown $N^{5,6}$, Hildebrand $M^{5,8}$, Abou Jamra $R^{2}$

${ }^{1}$ Austin Health, Melbourne, Australia

${ }^{2}$ Institute of Human Genetics, University Medical Center, Germany

${ }^{3}$ HudsonAlpha Institute for Biotechnology, Huntsville, USA

${ }^{4}$ Greenwood Genetics Center

${ }^{5}$ University of Melbourne, Australia

${ }^{6}$ Victorian Clinical Genetics Service, Parkville, Australia

Department of Pediatrics, University of Heidelberg, Germany

${ }^{8}$ Epilepsy Research Centre, Austin Health, Heidelberg, Australia

9 Joe DiMaggio Children's Hospital, Hollywood, USA

${ }^{10}$ University of Miami, USA

${ }^{11}$ Riley Hospital for Children, Indianapolis, USA

${ }^{12}$ UCSF Benioff Children's Hospital, San Francisco, USA

${ }^{13}$ Biochemistry Institute, Friedrich-Alexander-University of Erlangen-Nürnberg, Erlangen, Germany

Objective: Intellectual disability (ID) and autism are etiologically heterogeneous and the genetic diagnostic yield in patients with either phenotype improves with increasing disease severity. Whole genome sequencing in over 6,500 families from multiple populations with autism spectrum disorder (ASD) recently identified WDFY3 as a candidate ASD-risk gene, and WDFY3 is enriched for de novo loss of function (LoF) variants in 10,927 ASD/ID/DD patients. We describe the clinical and molecular details of patients with WDFY3 variants that were de novo or considered to be highly clinically relevant. Patients and Methods: Ten unrelated individuals with neurodevelopmental disorders were identified with heterozygous missense or LoF (nonsense, frameshift, splice acceptor, and splice donor variants) variants in WDFY3. Results: There were four individuals with a de novo LoF variant, one individual with a paternally-inherited LoF variant whose father had a history of learning difficulty, three with a de novo missense variant, and two individuals with a missense variant of unknown inheritance. Clinical characterization revealed an overlapping phenotype of neuro-psychiatric problems (ASD, ADHD, and anxiety disorders), developmental delay, intellectual disability, and macrocephaly. WDFY3 is highly expressed in the developing central nervous system and is important for neuronal connectivity, axon guidance, and the establishment of the major forebrain commissures. Null mutation in the mouse ortholog results in a comparable phenotype with neurodevelopmental deficits and macrocephaly. Conclusions: Based on the available clinical information and genetic results, individuals with heterozygous LoF variants in WDFY3 can develop macrocephaly, intellectual disability, and ASD.

\section{HOW DIFFERENTIAL TRANSCRIPTION CORRELATES WITH PATHOLOGY ACROSS THE CENTRAL NERVOUS SYSTEM IN AMYOTROPHIC LATERAL SCLEROSIS}

Watson $\mathrm{O}^{\prime}$, Williams $\mathrm{K}^{\prime}$, Hogan $\mathrm{A}^{\prime}$, Zhang K', Tarr I', Rowe D', Blair I'

${ }^{1}$ Macquarie University, Sydney, Australia

Amyotrophic lateral sclerosis (ALS) is a complex neurodegenerative disease characterized by progressive degeneration of motor neurons, leading to muscle atrophy and death. Genetic mutations are the only established cause of ALS. However, approximately $90 \%$ of Australian ALS cases do not harbour a known causal mutation. Disease discordance is observed between monozygotic twins as well as between family members with identical causal mutations. This indicates that environmental factors likely contribute to pathogenesis through epigenetic mechanisms. We aim to assess how differential gene expression correlates with disease pathology. To do this we will characterise the transcriptomes of seven central nervous system (CNS) regions that show differing levels of ALS pathology, as well as whole blood. Tissue samples from 22 ALS patients and 10 age/sex-matched controls were obtained from the Sydney and NSW Brain Banks. Pre-mortem blood samples, available for 10 of the 22 ALS cases, were collected from the Macquarie University MND biobank. RNA-sequencing will be utilized to generate transcriptomes for each individual sample. Genes that are differentially expressed between the CNS regions will be identified using student t-tests. Hierarchical clustering will be used to visualise sample similarity, and principal components analysis will reveal variables that characterise sample groups. The identification of an ALS associated transcriptomic profile in affected CNS regions may uncover new ALS gene candidates and enhance our understanding of the early molecular changes contributing to pathogenesis. If this profile is found in matched pre-mortem blood, it may lead to the development of a widely applicable transcriptome-based blood biomarker.

\section{NEXT GENERATION SEQUENCING PANEL DETECTS STRUCTURAL VARIANTS OF THE RB1 GENE IN A TUMOR SAMPLE}

Wells $A^{\prime}$, Soubrier $J^{\prime}$, Gao $S^{1}$, Henry $D^{\prime}$, Kenyon $R^{2}$, Lin $M^{2}$, Nataren $N^{2}$, Rossini $J^{1}$, Vakulin $C^{1}$, Wang $P^{2}$, Geoghegan $J^{2}$, Foresto $S^{3}$, Gole $G^{3}$, Susman $R^{4}$, Scott $H^{1}$, Kasshan K', Rawlings L'

${ }^{\prime}$ Genetics \& Molecular Pathology, SA Pathology, Adelaide, Australia

${ }^{2}$ ACRF Cancer Genome Facility, Centre for Cancer Biology, Adelaide, Australia

${ }^{3}$ Department of Ophthalmology, Lady Cliento Children's Hospital, Brisbane, Australia ${ }^{4}$ Genetic Health Queensland, Royal Brisbane and Women's Hospital, Herston, Australia

Aberrations of the RB1 and MYCN genes have been reported in the literature in association with retinoblastoma. Comprehensive screening of blood and tumor samples yields a detection rate of $>90 \%$ of patients presenting with retinoblastoma. We report a case of a 3 year old female presenting with unilateral retinoblastoma of the right eye. Standard analyses of DNA extracted from fresh tumor showed no somatic variants detected through NGS panel testing, an inconclusive $\mathrm{LOH}$ result and a negative hypermethylation result. Close examination of the NGS sequence data in IGV revealed two unexpected abnormalities; a somatic heterozygous deletion of RB1 exons 19 to 27 and a somatic structural variant with a breakpoint located in RB1 intron 4 adjacent to exon 5. Reads spanning this breakpoint also map to intron 2 of the PCDH9 gene, which is located $18 \mathrm{Mb}$ downstream on chromosome 13. Bioinformatics $\mathrm{CNV}$ analysis confirmed the deletion and showed a decrease in coverage over RB1 exon 5. No abnormalities were detected in the blood sample, indicating both events have occurred somatically. This case study shows the benefit of closely interrogating NGS data of retinoblastoma samples in detecting unusual abnormalities of the RB1 gene and the value of additional bioinformatics pipelines to detect structural abnormalities in routine analyses. This is important in being able to provide a conclusive report to the requesting practitioner and patient to define cancer predisposition risks to the patient and relatives.

\section{PARTIAL 70 DELETION IN A PRENATAL SAMPLE: CASE STUDY AND REVIEW}

Wilkin $\mathrm{H}^{\prime}$, Krishnaswamy R, Kulkarni A

${ }^{I}$ Monash Health, Clayton, Australia

Deletion of the distal region of the long arm of chromosome 7 is a rarely diagnosed abnormality with a varied and complex phenotype. Most deletions are terminal with breakpoints at $7 \mathrm{q} 35$ or $7 \mathrm{q} 36$, and include the $\mathrm{SHH}$ (sonic hedgehog) gene located at 7q36.3. This gene is important in development of the brain, central nervous system, eyes and limbs. Monosomy for this gene can cause 
holoprosencephaly which, in addition to hydronephrosis, growth retardation and sacral agenesis, is a commonly described feature of this chromosomal abnormality. The majority of deletions are de novo, although some have been identified as a result of malsegregation of a parental translocation involving chromosome 7. An amniocentesis was performed following detection of short long bones, hydronephrosis and coarctation of the aorta on ultrasound scan. Holoprosencephaly was not detected. CGH-microarray analysis revealed a copy number loss of 7q36.1 to 7q36.3. Genotype-phenotype correlations between this case and other published cases will be discussed.

\section{VALIDATION OF THE ILLUMINA CYTOSNP-12 PLATFORM TO DETECT COPY NUMBER CHANGES IN CHRONIC LYMPHOCYTIC LEUKEMIA}

Wilson $C^{\prime}$, Throp $D^{\prime}$, Harvey $Y^{\prime}$, Harraway J', Barton $N^{\prime}$, Bhuimbar $M^{\prime}$, Warner $M^{\prime}$, Brookwell $\mathrm{R}^{\mathrm{I}}$

${ }^{I}$ Sullivan Nicolaides Pathology, Bowen Hills, Brisbane, Australia

Chronic lymphocytic leukemia (CLL) is the most common leukemia in the Western world and is characterized by an accumulation of malignant B-lymphocytes in lymphoid tissue, marrow and blood. Despite remarkable genetic heterogeneity and largely sporadic occurrence, the majority of newly diagnosed CLL patients have recurrent chromosomal abnormalities. Current CLL diagnostic practice at Sullivan Nicolaides Pathology utilises cytogenetic information obtained through fluorescence in situ hybridization (FISH) and conventional karyotyping to help guide treatment regimes. Importantly, the emergence of prognostic CLL biomarkers and movement towards genetics-based disease stratification indicates a more specific method of genetic testing is required. Here we assess the use of the Illumina CytoSNP-12 microarray platform to detect copy number changes at genomic sites implicated in CLL, using DNA extracted from peripheral blood $(\mathrm{PB})$ and bone marrow $(\mathrm{BM})$ specimens. These findings were then compared to the corresponding karyotype and/or CLL FISH panel result where available. We found the microarray platform was effective in detecting all relevant abnormalities in PB and BM samples where the abnormal cell population was above the established limit of sensitivity for single nucleotide polymorphism (SNP) microarrays in the literature. Discrepancies between karyotype, FISH and microarray results are discussed, with emphasis on the benefits of using a diagnostic and prognostic multidisciplinary approach to CLL genetic testing.

\section{BALANCED TRANSLOCATION DISRUPTING SCN5A IN A FAMILY WITH BRUGADA SYNDROME AND SUDDEN DEATH}

Yeates L $^{1,3}$, Ingles $\mathrm{J}^{1,2,3}$, Gray $\mathrm{B}^{1,2,3}$, Semsarian $\mathrm{C}^{1,2,3}$, Bagnall $\mathrm{R}^{1,2}$
${ }^{1}$ Agnes Ginges Centre for Molecular Cardiology, Centenary Institute, Newtown,
Australia
${ }^{2}$ Sydney Medical School, University of Sydney, Sydney, Australia
${ }^{3}$ Department of Cardiology, Royal Prince Alfred Hospital, Sydney, Australia

Brugada syndrome $(\mathrm{BrS})$ is a rare primary arrhythmia syndrome. $\mathrm{Up}$ to $25 \%$ of familial $\mathrm{BrS}$ have a pathogenic variant in the sodium voltage-gated channel alpha subunit 5 gene (SCN5A). Balanced translocations occur in 1 in 500 of the general population. Family TD presented after the sudden death of their son, age 20 years. Post-mortem examination revealed asymmetric left ventricular hypertrophy and histology consistent with possible hypertrophic cardiomyopathy. Clinical screening of the family with echocardiogram and standard ECG was normal; however, a balanced translocation $\mathrm{t}(3 ; 10)(\mathrm{p} 21.3 ; \mathrm{p} 13)$ was previously found in the deceased's mother. Eight years after the death, the deceased's brother was diagnosed with BrS following a syncopal episode. Clinical screening of his half-sister revealed a BrS diagnosis and their mother was diagnosed with sick sinus syndrome. Sequencing a 128 cardiac gene panel was negative. Repeat karyotype of the mother revised the translocation breakpoints to $\mathrm{t}(3: 10)(\mathrm{p} .22 .2 ; \mathrm{p} 13)$, at the SCN5A locus. The translocation was present in the brother and half-sister. Fluorescent in-situ hybridization confirmed disruption of SCN5A, and whole genome sequencing (WGS) located the translocation breakpoint within intron 6 of SCN5A. Amplification of the deceased's DNA confirmed he also harboured the translocation, which we classified as pathogenic for BrS using ACMG criteria. This is the first description of a balanced translocation causing $\mathrm{BrS}$, and disrupting SCN5A. Our case highlights the importance of review of all available clinical history and genetic information within a specialized multi-disciplinary cardiac genetic clinic, and that WGS can detect and pinpoint the breakpoints of chromosomal translocations.

\begin{tabular}{l}
\hline NEWBORN SCREENING \\
\hline 160. EXOME SEQUENCING IN NEWBORNS WITH \\
CONGENITAL DEAFNESS AS A MODEL FOR GENOMIC \\
NEWBORN SCREENING \\
\hline Downie $\mathrm{L}^{1,2,3,3,4}$, Halliday $\mathrm{y}^{2,4}$, Jarmolowicz $\mathrm{A}^{1,6}$, Brett $\mathrm{G}^{1,6}$, Prawer $\mathrm{Y}^{5,6}$, Hunter $\mathrm{M}^{5}$, \\
\hline Lynch $\mathrm{E}^{2,6}$, Martyn $\mathrm{M}^{2,6}$, Forbes $\mathrm{E}^{2,6}$, Gaff $\mathrm{C}^{2,4,6}$, Amor $\mathrm{D}^{1,2,3,4}$ \\
${ }^{1}$ Victorian Clinical Genetics Service, Parkville, Australia \\
${ }^{2}$ Murdoch Children's Research Institute, Parkville, Australia \\
${ }^{3}$ Royal Children's Hospital, Parkville, Australia \\
${ }^{4}$ University of Melbourne, Melbourne, Australia \\
${ }^{5}$ Monash Health, Melbourne, Australia \\
${ }^{6}$ Melbourne Genomics Health Alliance, Melbourne, Australia
\end{tabular}

Background: Exome sequencing has the potential to be used in newborn screening and international research is investigating the feasibility of this approach. It is not yet clear how parents will respond to the genomic newborn screening in their children. Aim: To explore parents' interest in genomic testing and what factors influence decision making about the scope of results received. Methods: Families that consented for WES to identify the cause of hearing loss in their child were eligible. They could choose additional analysis of selected childhood onset genetic conditions unrelated to hearing loss. Families were provided with a decision aid and a genetic counseling session over two sites. Participants could choose to receive results for childhood onset genetic conditions with a known treatment or intervention pathway (Choice B), or all conditions, some of which do not have a known treatment or intervention pathway (Choice $C$ ). The families' completed evaluation surveys regarding their decision making post recruitment and at return of results. Results: Of the 107 patients who consented for WES, $64 \%$ opted to receive additional findings. Of those, $42 \%$ selected Choice B and $58 \%$ Choice C. Decisional conflict was lower among those who chose additional findings. The rate of uptake is significantly different between the two recruitment sites. Discussion: A majority of parents are requesting additional information. Analysis of genetic counseling notes and patient demographics will provide insight into parents' decision making. Conclusion: The results of this project illustrate the importance of providing choice around additional findings in genomic testing.

\section{ADVANCES IN PRENATAL SCREENING PROVIDE MOLECULAR CYTOGENETIC CHARACTERIZATION OF AN INSERTIONAL TRANSLOCATION: INS $(10 ; 7)(023 . ? 2 ; 031.3 ? 1036.1)$}

Gough $\mathrm{K}^{\prime}$, Pinto J', Pertile $\mathrm{M}^{2}$, Joy $\mathrm{C}^{\prime}$

${ }^{I}$ Mater Pathology-Cytogenetics Laborataory, Woolloongabba, Australia

2 Victoria Cancer and Genetic services, Melbourne, Australia

Insertional translocations are considered rare events that require three breaks within the chromosomes involved. In a simple oneway interchromosomal insertion, the first two breaks release an interstitial segment of a chromosome which is then inserted into the gap created by a third break in the second chromosome resulting 
in a complex chromosomal rearrangement. With the advent of new technologies for prenatal screening such as chromosomal microarray analysis, NIPT (Non-invasive prenatal testing), and recently whole-exome sequencing, previously undetectable insertions (de novo and familial) are being observed with more accurate sizing of the segments involved. Recent studies also suggest that the molecular mechanism may be more complex suggesting the involvement of nonhomologous end joining(NHEJ).The risk recurrence for a de novo interstitial loss and gain is low, however if a parent is a carrier of a balanced insertional translocation(IT) the risk is $50 \%$. We present a case of an unbalanced complex chromosomal rearrangement found in a prenatal chorionic villus sample involving chromosomes 7 and 10 where the parent was believed to have a balanced reciprocal translocation. This was identified previously by conventional cytogenetics. We show how emerging technologies such as NIPT and SNP microarray have provided further information indicating an insertional translocation and delineating more accurate breakpoints.

\section{IMPACT OF DEMOGRAPHIC FACTORS ON NEWBORN SCREENING BLOOD SPOT TSH LEVELS}

\footnotetext{
Heather $N^{\prime}$, Webster $D^{1}$, Derraik J ${ }^{2}$, Hofman $\mathrm{P}^{2}$

${ }^{I}$ NZ Newborn Metabolic Screening Programme, New Zealand

${ }^{2}$ Liggins Institute, University of Auckland, New Zealand
}

British newborn screening data have demonstrated that mean TSH values are increased among Pakistani, Bangladeshi and Chinese babies. We aimed to determine the influence of ethnicity and other demographic variables on New Zealand newborn screening TSH levels. Retrospective population study using 374,033 blood spot TSH cards received by the national newborn screening programme between 2010 and 2015. Blood spot TSH results and demographic data were recorded. The main outcome measure was the proportion of samples exceeding different TSH cut-offs (TSH $\geq 5, \geq 10$ and $\geq 15$ $\mathrm{mIU} / \mathrm{l})$. Pacific Island, Maori and Chinese babies were more likely to exceed TSH cut-off than New Zealand European babies at all thresholds. The odds ratio of exceeding TSH cut-off was highest in Pacific Island babies and was 1.8 at TSH $\geq 5(p<.0001), 2.4$ at TSH $\geq 10$ $(\mathrm{p}<0.0001)$ and 2.1 at TSH $15 \mathrm{mIU} / \mathrm{l}(p<.012)$. Higher levels of socioeconomic deprivation, male gender, and younger age at sample collection were also independently associated with greater odds of exceeding TSH cut-offs at $\geq 5$ and $\geq 10 \mathrm{mlU} / \mathrm{l}$. Newborn screening TSH levels vary depending on ethnicity, gender, deprivation and baby age at sampling. Population characteristics impact the reliability of screening and should be considered when setting and interpreting TSH cutoffs.

\section{IMPROVING THE SPECIFICITY OF TANDEM MASS SPECTROMETRY NEWBORN SCREENING}

Wong $D^{\prime}$, Tzanakos $N^{\prime}$, Wimalaratna $S^{\prime}$, Pitt J' ${ }^{I}$ Victorian Clinical Genetics Services, Murdoch Children's Research Institute,
Melbourne, Australia

Multiple reaction monitoring transitions used in tandem mass spectrometry newborn screening are not always completely specific and interferences sometimes occur. A known example is OHPro interference in Xleu. We also detected significant interference in C5:1 carnitine measurement, up to the equivalent of $1 \mu \mathrm{mol} / \mathrm{L}$, in some samples using a $300>85 \mathrm{~m} / \mathrm{z}$ transition. The resulting tail on the population distribution contributed to a relatively high cut-off of 8.0 multiple of median in our program. C5:1 carnitine is a marker for beta ketothiolase and 2-methyl-3-hydroxybutyryl-CoA dehydrogenase deficiencies. However, increases in this marker can be subtle in some patients and potentially masked by this interference. We used a second qualifier $300>104 \mathrm{~m} / \mathrm{z}$ transition to identify samples containing the interference. This allowed us to minimise the number of samples requiring second-tier testing as well as decreasing the C5: 1 carnitine cut-off to improve the detection of disorders associated with this metabolite. Such qualifier transitions can be a useful adjunct to routine mass spectrometry procedures to improve newborn screening specificity even when the interference has not been identified.

\section{NEWBORN SCREENING FOR PRIMARY IMMUNODEFICIENCIES - A METHOD EVALUATION}

Esber $\mathrm{R}^{\prime}$, Theresa $\mathrm{S}^{1}$, Hjort $\mathrm{M}^{3}$, Wiley $\mathrm{V}^{1,2}$

${ }^{I}$ NSW Newborn Screening Programme, Westmead, Australia

${ }^{2}$ University of Sydney, Sydney, Australia

${ }^{3}$ Perkin Elmer, Turku, Finland

Primary immunodeficiencies (PIDs) including severe combined immunodeficiency (SCID) are a group of disorders with an absence of humeral and cellular immunity. PIDs are caused by genetic defects which affect the development of both $\mathrm{T}$ and B lymphocytes. Screening tests for SCID measure T-cell receptor excision circles (TREC), which are in abundance in healthy newborns, and a similar test for kappa-deleting recombination excision circles (KREC) can indicate other PIDs. Infants appear normal at birth and without treatment usually die in the first year of life. Effective treatments including bone marrow transplant make this group of deficiencies suitable candidates for newborn screening. A PCR and time-resolved fluorescence resonance energy transfer assay kit (Enlite TREC-KREC kit, Perkin Elmer) was evaluated using a cohort of 2000 de-identified dried blood spot samples collected for routine newborn screening (NBS) with parental research consent. Archival samples from 20 proven SCID and 20 other PID patients and aged matched controls were also assayed. Results were quantitated using a calibration curve and validity of each batch was determined by quality control samples supplied with the kit. The method was found suitable for screening purposes with a maximum of $8 \times 96$ well plates analyzed per day per analyst. All NBS samples had a TREC and KREC value $>20$ copies/ul with median values of 153 copies/ul and 187 copies/ul respectively. All 40 deficient patients were detectable with very low to absent TREC/KREC values. The measurement of TREC and KREC provides detection of babies with PIDs in a timely manner to allow early treatment.

\section{SCREENING FOR 139 KNOWN CYSTIC FIBROSIS VARIANTS USING TARGETED NEXT GENERATION SEQUENCING}

Kim W', Bennetts $\mathrm{B}^{2,3}$, Wiley $\mathrm{V}^{1,3}$

${ }^{I}$ NSW Newborn screening Programme, Westmead, Australia

${ }^{2}$ Sydney Genome Diagnostics, Westmead, Australia

${ }^{3}$ University of Sydney, Sydney, Australia

Background: Cystic fibrosis (CF) has been included in routine dried bloodspot screening (DBS) in NSW since 1981 and has an incidence of 1:3000 live births. Since 1993 the screening protocol included DNA variant analysis for the commonly occurring variants. The most common, detected on at least one allele in over $90 \%$ of patients is p.F508del while other variants are detected on $<5 \%$ of alleles. As CF symptoms develop early and are life-threatening, rapid identification of those with CF is required. In NSW, next-generation sequencing (NGS) technology was evaluated for detection of 139 CF mutations in dried blood spots using a recently available kit from Illumina. Method: Genomic DNA was isolated from a $3.2 \mathrm{~mm}$ punch from DBS of 77 proven CF patients. Each DBS was incubated with DNA elution buffer (Wisconsin Newborn Screening Program). The library preparation was performed by using MiSeqDx $\mathrm{CF}$ assay reagents (Illumina). The MiSeqDx CF 139-Variant Assay was sequenced on the MiSeqDx instrument using a paired-end 150 cycles run with dual indexing. The whole blood extracted genomic DNA of 9 proven CF patients was also performed on the 
MiseqDX instrument. Results: The NGS assay was performed well using DNA isolated from DBS. The MiseqDX and current NBS technique (high resolution melting analysis) gave $100 \%$ concordant results. All previously established CFTR mutations were also detected in the whole blood extracted genomic DNA. Conclusion: The NGS assay provides detection of additional mutations allowing the reduction in time to treatment for babies with $\mathrm{CF}$.

\section{NEWBORN SCREENING FOR SPINAL MUSCULAR ATROPHY}

Theresa $\mathrm{S}^{1}$, Wotton $\mathrm{T}^{1}$, Wiley $\mathrm{V}^{1,2}$

${ }^{I}$ Sydney Children's Hospital Network, Wentworthville, Australia

${ }^{2}$ University of Sydney, Sydney, Australia

Spinal muscular atrophy (SMA) is an autosomal recessive neuromuscular condition characterized by deletion of SMN1 gene that leads to progressive muscle wasting and death. There are four types of SMA which are categorized based on age of onset and achieved motor function. Type I patients present clinical signs before 6 months of age and, when untreated, are unable to live past the age of 2 years. Type II patients achieve the ability to sit and some may be able to stand with the age of onset between 7 to 18 months. SMA Type III patients, known as mild SMA, achieve the ability to stand and walk during adulthood while SMA Type IV is also known as adult-onset SMA ( $>18$ years). With estimated incidence of 1 in 10,000 and carrier frequency of 1 in 50, SMA is one of the most common fatal genetic disorders. Using the same dried blood spot collected for newborn screening, SMN1 and SMN2 gene copy number can be successfully quantified using real time PCR with reported sensitivity and specificity nearly $100 \%$. Taking into consideration required automation, labor and consumable costs for this assay, routine screening for SMA is feasible. Recent trials in gene therapy have shown promising results in treating type I SMA patients if started before 12 weeks of age. Therefore, it is imperative to identify infants prior to presentation of symptoms. This study showed that screening for SMA fulfils all Wilson-Jungner criteria and is thus suitable for newborn screening.

\section{PRENATAL DIAGNOSIS}

\section{AN AUDIT OF PREGNANCY OUTCOMES FOLLOWING AN INCREASED RISK NIPT FOR XXX, XXY AND XYY}

\footnotetext{
Hunt $C^{1,2}$, Scarff $K^{1,2}$, Archibald $A^{1,2,3}$, Flowers $N^{1,2}$, Giouzeppos $O^{1,2}$, Delatycki $M^{1,2,3}$, Pertile $M^{1,2,3}$

${ }^{I}$ Victorian Clinical Genetics Services, Parkville, Melbourne, Australia

${ }^{2}$ Murdoch Children's Research Institute, Parkville, Melbourne, Australia

${ }^{3}$ Department of Paediatrics, University of Melbourne, Parkville, Melbourne, Australia
}

The introduction of non-invasive prenatal testing (NIPT) allows screening for sex chromosome aneuploidy (SCA) during pregnancy. Victorian Clinical Genetics Services (VCGS) has offered perceptTM NIPT since April 2015 which includes routine screening for SCA. An audit was conducted to determine the outcome of pregnancies where an increased risk SCA was identified: triple $\mathrm{X}(47, \mathrm{XXX})$, Klinefelter syndrome $(47, \mathrm{XXY})$ and $47, \mathrm{XYY}$. The outcomes of high risk monosomy $\mathrm{X}$ results were excluded. Of 32,920 NIPT samples analyzed between April 2015 and August 2017, $72(0.22 \%)$ increased risk SCA results for 47,XXX, 47,XXY and $47, \mathrm{XYY}$ were issued. Where possible, outcome data from prenatal, postnatal and/or maternal chromosome investigations were collected. Outcome data were available for 54/72 (75\%) high risk SCA NIPTs, which confirmed 15/20 (75\%) 47,XXX, 17/22 (77\%) 47,XXY and 12/12 (100\%) 47,XYY cases. More severe SCAs were diagnosed (49,XXXXX and 48,XXYY) in two cases. Three high risk XXY results were due to maternal $Y$ chromosome copy number variants $(\mathrm{CNVs})$. Causes of false-positive results include maternal triple X, maternal and fetal CNVs, confined placental mosaicism, and statistical false-positives with no biological cause. Issues to consider in the provision of genetic counseling for an increased risk $47, \mathrm{XXX}, 47, \mathrm{XXY}$ or $47, \mathrm{XYY}$ NIPT result include prenatal screening for conditions with milder phenotypes compared to the common autosomal trisomies, decision making regarding prenatal diagnostic testing, and that the NIPT result is not always concordant with the final outcome. This audit allows greater insight regarding decisionmaking outcomes and test performance for 47,XXX, 47,XXY and 47,XYY SCA using perceptTM NIPT.

\section{THE COUNSELING IMPLICATIONS FOLLOWING PGS DIAGNOSIS OF A MOSAIC EMBRYO - TO TRANSFER OR NOT?}

Kliskey $\mathrm{N}^{\prime}$

${ }^{l}$ Monash IVF, Bardon, Australia

Background: Pre-implantation genetic screening (PGS) is now a frequently offered technique used to increase IVF pregnancy rates by identifying euploid embryos for transfer. Suitable embryos are biopsied at the blastocyst stage following 5-6 days in culture. Patients considering having the procedure are given counseling prior to their IVF treatment. The embryos are frozen following biopsy and screening is performed by Next Generation Sequencing. Results are reported 3 weeks after testing; either no abnormality detected, abnormal or mosaic. Recent changes to the PGD International Symposium guidelines on the fate of embryos diagnosed as mosaic have had significant implications on the counseling requirements before and after PGS. Case Study: A same-sex, donor-recipient couple presented for PGS on their 3rd treatment cycle. Results reported following embryo biopsy identified one complex abnormal embryo and one embryo that was reported as mosaic trisomy for the sex chromosomes. In accordance with clinic policy the mosaic embryo could be considered suitable for embryo transfer following adequate counseling. Discussion: This case presented several novel and unique counseling challenges. These included 3 potential genotypes and their clinical presentations, the unknown ratio of normal vs. abnormal cells and the potential varied distribution of these cells. The recommendation for prenatal testing was also discussed.

\section{THE LIVED EXPERIENCE OF DISABILITY, AND ATTITUDES TOWARDS DISABILITY, PRENATAL SCREENING AND TERMINATION OF PREGNANCY}

\footnotetext{
Long $\mathrm{S}^{1,2}, \mathrm{O}^{\prime}$ Leary $\mathrm{P}^{2}$, Dickinson $\mathrm{J}^{3}$

Genetic Services of Western Australia, Perth, Australia

${ }^{2}$ Curtin University, Perth, Australia

${ }^{3}$ King Edward Memorial Hospital, Perth, Australia
}

With emerging new prenatal screening technology women will potentially have a choice of a large range of genetic conditions to screen during a pregnancy, in addition to trisomy 13,18 and 21. Laboratories have begun to offer prenatal non-invasive full genome microarray and expanded cell free DNA screening which raise many questions about how women will manage this information and the impact on decision making in pregnancy. We conducted qualitative interviews to explore women's attitudes and knowledge towards termination of pregnancy (TOP), and their attitudes towards prenatal screening for congenital genetic anomalies, based on their lived experiences of disability. The three cohorts interviewed consisted of healthy women without children, healthy women with healthy children, and healthy women with children who have de novo genetic disorders. In response to open ended questions, the women described their lived experience of disability and whether TOP was a valid choice given different chances of physical or intellectual disability. The women discussed the types of information needed to make a decision about TOP for fetal conditions, and this was 
often influenced by their lived experience of disability. In general, the women were pro-choice (promoting autonomy) and the majority in each cohort considered a severe physical disability to be preferable to a severe intellectual disability. Given that prenatal microarray can detect copy number variants of unknown significance and expanded cell-free DNA screening has limited prediction of phenotypic outcome, decision-making is becoming more difficult and women require greater counseling support to implement their autonomous choices.

\section{SIMULTANEOUS DETECTION OF CHROMOSOMAL ABNORMALITIES AND MONOGENIC DISEASES IN EMBRYOS USING A NOVEL PGT METHOD}

\author{
Xia J $J^{2}$, Yang $L^{\prime}$, Chen $D^{\prime}$, Zhang $H^{2}$, Zhang $L^{\prime}$, Liu $P^{\prime}$, Chen $F^{1,2}, G a o Y^{2}$ \\ ${ }^{1}$ MGI, BGI-Shenzhen, Shenzhen, China \\ ${ }^{2}$ Institute of Maternal and Child Health, BGI-Shenzhen, Shenzhen, China
}

Background: Preimplantation genetic testing (PGT) helps patients select embryos that are free of monogenic diseases and chromosomal abnormalities. Next generation sequencing (NGS) has been used widely to detect chromosomal abnormalities and monogenic diseases in PGT. However, due to differences in current experimental procedures used to interrogate both types of changes, chromosomal abnormalities and monogenic diseases cannot be detected simultaneously. Aim: We developed a new method called the Targeted And Genome-wide simultaneous sequencing (TAGs-seq) which can be used to simultaneously detect chromosomal abnormalities and monogenic diseases. Methods: TAGs-seq identifies genetic abnormalities by using multiplex PCR to amplify target regions as well as whole genome sequencing. We validated TAGs-seq using $16 \mathrm{em}-$ bryo samples from two families affected by $\beta$-Thalassemia. Multiplex PCR primers for the HBB gene mutation known to cause this disease and for SNPs flanking this gene were designed. Following TAGs-seq, the HBB mutation was detected directly, and haplotypes which are linked to it were analyzed by linkage analysis to confirm the detected results. Copy number variants (CNVs) were detected using the whole genome sequencing data. Results: We generated over $500 \times$ depth data of the HBB gene mutation and flanking SNPs and $0.1 \times$ depth data covering the whole genome using BGISEQ-500 platform. Four of the 16 embryos were found to be free of mutated HBB, eleven embryos were found to be carriers of $\beta$ thalassemia, and one embryo was affected. Only one embryo, identified as a carrier, had CNVs larger than $16 \mathrm{Mb}$. All results were consistent with results obtained from the current gold standard methods. Conclusions: Our method, TAGs-seq, can detect both monogenic diseases and chromosomal abnormalities simultaneously with high precision.

\section{PUBLIC HEALTH GENETICS}

\section{IMPLEMENTATION SCIENCE IN GENOMICS:} BRIDGING RESEARCH AND CLINICAL PRACTICE

$\underline{\text { Best }}^{1,3}$, Long J', Taylor $\mathrm{N}^{2}$, Martyn $\mathrm{M}^{3,4,5}$, Braithwaite J', Gaff $\mathrm{C}^{3,4,5}$

${ }^{I}$ Australian Institute of Health Innovation, Macquarie University, Sydney, Australia

${ }^{2}$ NSW Cancer Council, Sydney, Australia

${ }_{3}^{3}$ Melbourne Genomics Health Alliance, Melbourne, Australia

${ }^{4}$ Murdoch Children's Research Institute, Melbourne, Australia

${ }^{5}$ Department of Paediatrics, University of Melbourne, Melbourne, Australia

Background: Implementation science studies how research gets into frontline clinical care. As genomics moves from bench-based research to the bedside the need to understand the process of translation becomes more significant. Australian and Melbourne Genomics have placed emphasis on researching the long-term sustainability of genomics in the clinical setting through their use of clinical projects. Aim: To investigate organizational and clinical level barriers and enablers to implementing genomic testing. Methods: Capability, op- portunity and motivation are known to influence the uptake of behavior (Michie et al., 2011). To examine these areas we designed two interview schedules. Thirty-two service level leads and 27 nongenetic medical specialists involved with Melbourne and Australian Genomic clinical projects, fulfilling the interview inclusion criteria, were identified and invited to interview. Twenty-one service level and 16 clinicians responded and were interviewed in 2018. Results: Our findings show the uptake of genomics is hindered by a preference (by some) to continuing research in genetic discovery rather than application in clinical practice (motivation), absence of a sustainable model of funding (opportunity) and unrealistic expectations about what genomics can deliver (motivation). The overriding enablers were the use or experience of genomic testing (opportunity) and the need to build relationships to develop genomic knowledge (opportunity). Conclusion: Learning from the experiences of service leads and clinicians currently using genomic testing will help identify approaches for future implementation. Synthesis of these data with clinical audit data will allow co-creation, with service leads and clinicians, of strategies to facilitate future implementation of genomics in clinical practice.

\section{FINDINGS FROM A CHURCHILL FELLOWSHIP COMPARING INNOVATIVE STRATEGIES FOR GENETIC COUNSELING}

Freeman $\mathrm{L}^{\prime}$

${ }^{I}$ Royal North Shore Hospital, St Leonards, Australia

Genomics has the potential to reshape clinical practice and to fundamentally change the way we prevent, diagnose, treat and monitor illness, providing the opportunity to have more precise and tailored treatments. The ability to respond to this change is dependent on further developing Australia's capacity, capability and infrastructure needed to integrate genomic technology into the health system. Genetic counselors will be integral to this process. This presentation will explore the findings from a comparative analysis of the US, the UK and Australia as identified on a recent Churchill Fellowship. Specifically, the provision of genetic counseling for patients accessing emerging genomic technologies was explored across the different sites. This report discusses international and institutional governance of genomic testing in the clinical setting and provides commentary and recommendations of best practice and models for delivery of genomic testing in Australia. The recommendations of this project were derived using the four principles underpinning the Genetic Counsellor Code of Ethics: Autonomy, non-maleficence, beneficence and social justice. Most ethical and social considerations raised by genomics already apply generally to health care. However, the application of genomic knowledge has its own particular challenges, including how the predictive nature of its application may impact on other family members, future generations and communities over time.

\section{PREVALENCE OF COMMON CHROMOSOMAL TRISOMIES AND THE ASSOCIATION BETWEEN SOCIOECONOMIC STATUS AND TIMING OF DIAGNOSIS}

Kluckow $E^{\prime}$, Halliday $\rfloor^{1,2}$, Poulton $A^{\prime}$, Lindquist $A^{1,4}$, Hui $L^{1,3,4}$

${ }^{I}$ Murdoch Children's Research Institute, Parkville, Australia

2 Paediatrics, University of Melbourne, Parkville, Australia

${ }^{3}$ Obstetrics and Gynaecology, University of Melbourne, Parkville, Australia

${ }^{4}$ Mercy Perinatal, Mercy Hospital for Women, Heidelberg, Australia

Aim: To use a state-wide perinatal dataset to evaluate prenatal and postnatal diagnoses of common autosomal trisomies in Victoria, and to explore the association between timing of diagnosis and socioeconomic status. Method: All Victorian women who received a diagnosis of trisomy 21 (T21), trisomy 18 (T18) or trisomy 13 (T13) in 2015-2016 were included. Prenatal diagnoses were 
obtained from amniocentesis and chorionic villus sampling results from the Victorian Prenatal Diagnosis database. Postnatal cytogenetic results from live infants, stillborn infants, and products of conception were obtained from the four Victorian cytogenetic laboratories. Socioeconomic status was assigned using the Index of Relative Socio-economic Advantage and Disadvantage (IRSAD) from maternal postcode. Results: There were 823 diagnoses of T21, T18, and T13 and 156,460 total births in Victoria. Of those, $577(70.1 \%)$ were $\mathrm{T} 21,139(16.9 \%)$ were T18 and $107(13.0 \%)$ were T13. Among the T21 group, $85(14.7 \%)$ were diagnosed in live infants. The remainder were diagnosed during pregnancy $(66.4 \%)$ or following perinatal loss $(18.8 \%)$. Among the T18 and T13 cases, only $1.4 \%$ and $2.8 \%$ of diagnoses were made in live infants respectively. In contrast, $>$ $97 \%$ of T18 and T13 cases were ascertained following perinatal loss. There was no significant difference in the proportion of cases diagnosed prenatally versus postnatally by IRSAD. Conclusion: This study provides up-to-date data on the state prevalence of major autosomal trisomies. T21 affects 1 in 278 pregnancies and 1 in 1852 live births. There was no significant difference in the timing of diagnosis according to socioeconomic status.

\section{CURRENT SITUATION OF NIPT AT A LOCAL PERINATAL MEDICAL CENTER IN JAPAN}

Kondo A', Nakaoku $D^{\prime}$, Yamasaki $M^{\prime}$, Morine $M^{\prime}$, Hinokio $K^{\prime}$, Maeda $K^{\prime}$

${ }^{I}$ Shikoku Medical Center for Children and Adults, Nho, Zentsuji, Japan

Introduction: In Japan, NIPT (non-invasive prenatal testing) have been introduced as clinical study in 2013, we also started NIPT in September 2013. It has been only our hospital offering NIPT in local area in our prefecture. We summarized the current status of prenatal test including NIPT around our area and make a comparative discussion with the data from NIPT consortium Japan. Method: In total, 666 women had genetic counseling for prenatal test including NIPT from 2013 to 2017. We analyzed the clinical data including their background, test results and pregnancy outcomes. Results: Overall, 613(92.0\%) women had NIPT and 53 women (8.0\%) did not. Among the 613 women tested, 15 cases were positive (2.45\%) for aneuploidy (T21:12 cases, T18:3 cases, T13:0case). Average age of 613 women who had NIPT was 38.1 years old. Reasons for having NIPT were advanced age ( $89.1 \%$ ), high risk of chromosomal aberrations with ultrasound $(6.5 \%)$, previous pregnancy with chromosome aberrations $(3.8 \%)$, positive result with maternal serum screening $(0.7 \%)$, respectively. One of three trisomy 18-positive patients was diagnosed as false-positive with amniocentesis. Conclusion: Positive result frequency was $2.45 \%$ and was higher than $1.8 \%$, NIPT consortium of Japan. This difference in positive rate could be related to the background of our patients such as high risk findings with ultrasound. We also consider low-risk patients tend to not seek for prenatal test around our area. In addition, we also showed ultrasound screening at 1 st trimester is important as reported by many people previously.

\section{PREPARING FOR GENOMICS: A RETROSPECTIVE AUDIT OF A METROPOLITAN RENAL GENETICS CLINIC}

Krzesinski $E^{1,2}$, Prawer $Y^{1,2}$, Regan $M^{\prime}$, Yeung $A^{1,2,3}$, Hunter $M^{1,2}$

${ }^{I}$ Monash Health, Melbourne, Australia

${ }^{2}$ Department of Paediatrics, Monash University, Melbourne, Australia

${ }^{3}$ Victorian Clinical Genetics Service, Murdoch Children's Research Institute, Melbourne, Australia

Background: Hereditary kidney conditions are rare but account for a significant burden on hospitals in both adults and children. Aim: Monash Health is a KidGen/ Australian Genomics Health Alliance/Melbourne Genomics Health Alliance flagship recruitment site, aiming to assess the impact of multidisciplinary renal-genetic clinics on patient care while providing access to whole exome sequencing (WES). Methods: A three-year audit (2015-2017) of re- nal patients referred to the Monash Health genetics service provides a picture of the renal genetics landscape at the outset of renal flagship WES testing. The electronic medical record was searched using renal keywords; each file was manually assessed for inclusion and exclusion criteria. Data collection included referrer specialty, condition, family history, inheritance pattern, type and cost of genetics testing and impact of genetic testing on management and reproductive options. Results: Through keyword matching, 676 patients were identified and 86 met inclusion criteria; 59 (69\%) were probands with a primary renal condition and $35(40.1 \%)$ were referred by a nephrologist. Cystic renal disease was the commonest reason for referral. Genetic testing was arranged for 50 patients of whom 21 (42\%) had informative results. Diagnosis rate improved from 2015 to 2017 ; cost per informative patient diagnosis was $\$ 2,499$. Conclusion: This audit provides a snapshot of the utility of clinical genetics input in a renal genetics context at the outset of improved WES access. Fourteen referred patients were unaffected, providing them with reproductive reassurance. Diagnosis rate improved from 2015 to 2017 as more molecular diagnoses were obtained, thus improving patient management.

\section{DESIGNING SYSTEMS TO SHARE CLINICAL GENOMIC SEQUENCING DATA FOR RESEARCH: INCLUDING THE PATIENT VOICE}

Martyn $M^{1,2,3}$, Gleeson $P^{4}$, Walker $C^{5}$, Chia $H^{4}$, Kanga-Parabia $A^{1,3}$, Gaff $C^{1,2,3}$

${ }^{I}$ Melbourne Genomics Health Alliance, Parkville, Australia

${ }^{2}$ Murdoch Children's Research Institute, Parkville, Australia

${ }^{3}$ The University of Melbourne, Parkville, Australia

${ }^{4}$ Melbourne Law School, The University of Melbourne, Parkville, Australia

${ }_{5}^{5}$ Chronic Illness Alliance, Surrey Hills, Sydney, Australia

Stored genomic data from clinical testing is potentially valuable for research. In contrast to community and research participant views, patient expectations about how clinically obtained genomic data is stored, used and shared for research have not been explored. We address this gap by examining the willingness of patients receiving genomic testing through clinical care to share their data and surveying them about their preferences for use of clinical data for research. Categorical data is analyzed using descriptive statistics, and openended questions using content analysis. To date, 99\% (975/989) of patients agreed to share their re-indentifiable data for research. The survey response rate is $652 / 907(72 \%)$. Despite the clinical community's concerns about effectiveness of pre-test counseling, $96 \%$ $(602 / 624)$ of respondents indicate they had enough information before making their decision. There was no clear preference about the level of control patients want to have over use of their data for research: blanket consent $(11 \%)$ or refusal $(1.5 \%)$ were rarely preferred but there was no clear preference between opt-in or opt-out for each study or ongoing opt-in with the option of recontact to change this $(25 \%, 23 \%, 39 \%)$.Patients were surprisingly supportive of sharing their data and even re-identification keys with a range of organizations. A minority $(5 \%, 22 / 411)$ felt it would be easy for their data to be reidentified and would be concerned if this occurred. Patient voices are informing data governance, design and prioritization of a single system for research access to state-wide clinical genomic sequencing data in Victoria.

\section{CONSUMER DRIVEN GENETIC TESTING: A NEW MODEL OF GENETIC COUNSELING FOR THE GENOMIC ERA}

Milgrom Z', Amor $\mathrm{D}^{1,2,3}$, Kalro $\mathrm{K}^{\prime}$, Lanyon $\mathrm{K}^{\prime}$

${ }^{1}$ Eugene Labs, Australia

${ }^{2}$ MCRI, Australia

${ }^{3}$ University of Melbourne, Australia

The genomic revolution is changing the way we understand risk, implement prevention programs and treat disease — the problem is that 
the majority of the population has no awareness of these changes in medical management and the gap in access to genetic testing risks creating inequality in health outcomes. Access to genetic testing historically has been offered in specialist genetic clinics and reserved primarily for individuals with suspected syndromes, family history of genetic disease or the wealthy early-adopters. Traditional genetic clinics are struggling to keep up with the increasing demand and GPs and sub-specialists don't have the scope to fill the void. Moreover the reputation and service offered by existing direct-to-consumer marketplaces has not provided a safe, ethical and reliable middle ground. As a result, there is a bias in access to genetic testing that leans towards high-income earners, Caucasians, and men. We have developed a model to address the changing market for genetic testing by combining the best of technology and the empathy of genetic counseling and will be offering ethical, actionable, culturally sensinetics clinic. The proposed model involves partnering with NATAaccredited lab leaders and providing a new open-platform for consumers to initiate genetic testing in a fully supported digital clinic powered by experienced genetic counselors. This app-based genetics clinic is set to launch in late 2018. tive and affordable genetic testing via a revolutionary app-based ge-

\section{THE DEVELOPMENT OF A NATIONAL CLINICAL CONSENT PACKAGE FOR GENOMIC TESTING}

\author{
Pereira $K^{1,2}$, Boggs $K^{1,3,4}$, Boughtwood $T^{1,2}$, Fletcher $J^{5,6}$, Haas $M^{1,2}$, Kaub $P^{7}$, \\ Lynch $E^{2,8,14}$, Macciocca $\mathrm{I}^{2,14}$, Mckenzie $\mathrm{F}^{9,10}$, Newson $\mathrm{A}^{1 \mathrm{l}}$, Phillips $\mathrm{P}^{1,2}$, Roscioli \\ $\mathrm{T}^{4,12}$, Ross $\mathrm{B}^{13}$, Wilson $\mathrm{M}^{3,15}$, Young $\mathrm{M}^{16}$, McGaughran $\mathrm{J}^{17}$ \\ ${ }^{1}$ Australian Genomics, Melbourne, Australia \\ ${ }^{2}$ Murdoch Children's Research Institute, Melbourne, Australia \\ ${ }^{3}$ Department of Clinical Genetics, The Children's Hospital Westmead, Sydney, \\ ${ }^{4}$ Centralia
${ }_{5}$ SA Por Clinical Genetics, Sydney Children's Hospital Randwick, Sydney, Australia \\ ${ }_{6}^{5}$ SA Pathology, Adelaide, Australia \\ ${ }^{6}$ University of Adelaide, Adelaide, Australia \\ ${ }^{7}$ Australian Clinical Laboratories, Clayton, Australia \\ ${ }^{8}$ Melbourne Genomics Health Alliance, Melbourne Genomics Health Alliance, \\ Australia \\ ${ }^{9}$ Genetic Services of Western Australia, King Edward Memorial Hospital, Subiaco \\ Australia \\ ${ }^{10}$ School of Paediatrics and Child Health, University of Western Australia, Perth, \\ Australia \\ ${ }^{11}$ Sydney Health Ethics, Sydney School of Public Health, University of Sydney, Sydney, \\ Australia \\ ${ }^{12}$ Neuroscience Research Australia, Sydney, Australia \\ ${ }^{13}$ The Royal College of Pathologists of Australasia, Sydney, Australia \\ 14 Victorian Clinical Genetics Services, Melbourne, Australia \\ ${ }^{15}$ Disciplines of Genetic Medicine and Paediatric \& Adolescent Health, University of \\ Sydney, Sydney, Australia \\ ${ }^{16}$ Genome.One, Sydney, Australia \\ ${ }^{17}$ Genetic Health Queensland, Brisbane, Australia
}

Genomic testing raises complex issues, with a number of possible testing outcomes and potential implications for the individual and their family. Providing patients with clear and concise information during the consent process is critical to support well-informed decisions about testing. Currently, genetic services within Australian states and territories take an individualized approach to clinical genomics consent. This can be a barrier to the flow of health information across jurisdictional borders and can confuse the interpretation of laws and policies about sharing genomic data for clinical or research purposes. The Australian Genomics National Consent Working Group was formed with the remit of undertaking a consultative process to inform the development of a clinical genomic consent form for national use across genetic conditions. Currently used clinical and research consent forms were evaluated by the Working Group, through a survey process. Survey data was used to draft a consent form which was reviewed by the working group. The second iteration of the form was reviewed by relevant organizations, including Consumer Advisory Groups and peak bodies - for example, HGSA. An information package to support the consent form was developed using similar iterative consultation processes. A concise consent form and a supporting information package were developed, which addresses areas identified by the group as essential and relevant to how genomic testing is offered. It explains to participants testing outcomes; data analysis; gene lists; further sample use and genomic data in research; data sharing, risks of genomic testing and familial implications and insurance.

\section{WHAT'S VALUE FOR MONEY: WHOLE-EXOME SEQUENCING OR WHOLE-GENOME SEQUENCING? COMPARISON FROM AN ECONOMIC PERSPECTIVE} synthesized and grouped. Results: (Please note figures may be revised slightly pending review) Nearly a third of the 96 initiatives reported involving people (30/96, 31\%), with nearly 1 in 3 of those initiatives reporting impacts from the involvement $(9 / 30,30 \%)$. We found significant variability in the type, frequency and methods of involvement reported. Some initiatives $(4 / 30,13 \%)$ reported involving people at every stage of the research cycle. Conclusion: There was a wide diversity in the methods and tasks of involvement, ranging from involvement in every stage of the research to more modest activities such as public consultations. Self-reported impacts were positive in their description, signalling the future benefits of involvement for improving public trust in genomics research.
Shrestha $R^{\prime}$, Schofield $D^{1}$, Ewans $L^{2,3}$, Field $M^{4}$, Dinger $M^{2,3}$, Buckley $M^{5}$, Roscioli $\mathrm{T}^{6}$

${ }^{1}$ Centre for Economic Impacts of Genomic Medicine, Department of Economics, Macquarie University, Australia

${ }^{2}$ St Vincent's Clinical School, University of New South Wales, Australia

${ }^{3}$ Kinghorn Centre for Clinical Genomics, Garvan Institute of Medical Research, Australia

Australia
${ }^{4}$ The Genetics of Learning Disability Service, Australia

${ }^{5}$ Randwick Genetics, NSW Health Pathology East, Prince of Wales Hospital, Australia ${ }^{6}$ NeuRA and Prince of Wales Clinical School, University of New South Wales, Australia

There is considerable evidence that using next-generation sequencing such as whole-exome sequencing (WES) or whole-genome sequencing (WGS) improves the diagnostic rate for genetic disorders, 
with studies reporting a higher diagnostic yield for WGS than WES when applied to Mendelian disorders. Despite a higher diagnostic rate for WGS, its higher cost compared to WES remains a barrier. There remains a lack of evidence on which of these two technologies is more cost-effective for genetic diagnosis. The main aim of this study is to analyze whether WGS or WES provides greater value for money, when applied to the diagnosis of Mendelian disorders. This study used data from a cohort of 100 individuals from 38 families with Mendelian disorders without molecular diagnosis after WES. They then have had WGS performed for a molecular diagnosis. This study will collect cost data using a bottom-up approach. We will compare the cost of four diagnostic pathways: primary WES analysis, WES reanalysis with current pipelines, primary WGS analysis after WES analysis fails to provide a molecular diagnosis and a counterfactual pathway of WGS replacing WES at the beginning of the diagnostic pathway. The cost-effectiveness of WGS replacing WES will be analyzed in terms of an incremental cost per additional diagnosis compared to WES. The results will identify the most costeffective diagnostic pathway for Mendelian disorders and enable the most efficient use of health-care resources.

\section{THE COST OF HOSPITALIZATION FOR CHILDHOOD CANCER AND DEVELOPING AN ECONOMIC MODEL OF PRECISION MEDICINE}

Tan $\mathrm{O}^{\prime}$, Schofield D', Shrestha $\mathrm{R}^{\prime}$

${ }^{I}$ Centre For Economic Impacts of Genomic Medicine, North Ryde, Australia

Cancer is the leading cause of death by disease among Australian children. Survivors of childhood cancer often suffer from comor- bidities such as osteoporosis, heart disease, and mental health issues. The aim of this study is to estimate the cost of childhood cancer related hospitalizations in New South Wales and to develop a modeling framework to analyze the impact of precision medicine in the treatment cost of childhood cancers. We used CHeReL linked datasets of children under 18 year of age, living in NSW, who have had cancer. Their records were extracted from the NSW Central Cancer Registry and were linked to RBDM death registrations, NSW Cause of Death Unit Record File, Admitted Patient Data Collection, and Emergency Department Data Collection. Individual patient's hospital services usage was determined from the hospital and emergency department datasets. Cost was determined using the price of an $\mathrm{ABF}$ activity published by the Independent Hospital Pricing Authority. Acute lymphoblastic leukemia, brain, Hodgkin's disease, non-Hodgkin's lymphoma, and bone cancer accounted for $50 \%$ of the reported incidence. The average number of hospital admissions per year per patient was 4 , and average emergency presentation was 1.9 times per year per patient. The estimated annual cost of hospitalization and emergency department presentations were $\$ 135,486$ and $\$ 1,234$ per patient respectively. The population within our dataset will serve as baseline measure, from which we will be able to simulate the impact of NGS and the precision medicine on individual pediatric patients, by sourcing data from published literature and clinical trials outcomes. 